ESAIM: M2AN 47 (2013) 1583-1626

DOI: $10.1051 / \mathrm{m} 2 \mathrm{an} / 2013077$
ESAIM: Mathematical Modelling and Numerical Analysis

www.esaim-m2an.org

\title{
DERIVATION OF LANGEVIN DYNAMICS IN A NONZERO BACKGROUND FLOW FIELD
}

\author{
Matthew Dobson ${ }^{3,4,1}$, Frédéric Legoll 2,3 , Tony Lelièvre ${ }^{3,4}$ \\ AND GabRIEL Stoltz ${ }^{3,4}$
}

\begin{abstract}
We propose a derivation of a nonequilibrium Langevin dynamics for a large particle immersed in a background flow field. A single large particle is placed in an ideal gas heat bath composed of point particles that are distributed consistently with the background flow field and that interact with the large particle through elastic collisions. In the limit of small bath atom mass, the large particle dynamics converges in law to a stochastic dynamics. This derivation follows the ideas of [P. Calderoni, D. Dürr and S. Kusuoka, J. Stat. Phys. 55 (1989) 649-693. D. Dürr, S. Goldstein and J. Lebowitz, Z. Wahrscheinlichkeit 62 (1983) 427-448. D. Dür,, S. Goldstein and J.L. Lebowitz. Comm. Math. Phys. 78 (1981) 507-530.] and provides extensions to handle the nonzero background flow. The derived nonequilibrium Langevin dynamics is similar to the dynamics in [M. McPhie, P. Daivis, I. Snook, J. Ennis and D. Evans, Phys. A 299 (2001) 412-426]. Some numerical experiments illustrate the use of the obtained dynamic to simulate homogeneous liquid materials under shear flow.
\end{abstract}

Mathematics Subject Classification. 82C05, 82C31.

Received March 16, 2012. Revised October 15, 2012.

Published online August 20, 2013.

\section{INTRODUCTION}

Molecular dynamics simulations have been increasingly used to bring atomistic accuracy to macroscopic fluid models $[10,20,21,28]$. One example is the computation of the constitutive relation between the strain rate $A=\nabla \mathbf{u}$ and the stress tensor $\boldsymbol{\sigma}(\nabla \mathbf{u}, T)$ in complex fluids at temperature $T$, where one uses a microscopic simulation to determine the closure relation for the continuum equations [16]. We thus wish to simulate molecular systems at temperature $T$ that are subject to a steady, nonzero macroscopic flow, and the goal of this paper is the derivation of a dynamics to sample such states.

Keywords and phrases. Nonequilibrium, Langevin dynamics, multiscale, molecular simulation.

1 Department of Mathematics and Statistics, 710 N. Pleasant Street, University of Massachusetts, Amherst, MA 01003-9305, USA. dobson@math. umass.edu

${ }^{2}$ Laboratoire Navier - Ecole des Ponts ParisTech, 6 et 8 avenue Blaise Pascal, Cité Descartes - Champs sur Marne, 77455 Marne la Vallée Cedex 2, France. legoll@lami.enpc.fr

3 INRIA Rocquencourt, MICMAC team-project, Domaine de Voluceau, B.P. 105, 78153 Le Chesnay Cedex, France

4 CERMICS - Ecole des Ponts ParisTech, 6 et 8 avenue Blaise Pascal, Cité Descartes - Champs sur Marne, 77455 Marne la Vallée Cedex 2, France. \{lelievre,stoltz\}@cermics.enpc.fr 
Simulation of molecular systems with a nonzero background flow is one goal of nonequilibrium molecular dynamics (NEMD) techniques [4,9]. Several strategies have been proposed to sample molecular systems under nonzero flow: for example, the SLLOD and g-SLLOD equations of motion $[8,9,26,27]$ or dissipative particle dynamics [24]. Typically, these are used in conjunction with consistent boundary conditions such as the LeesEdwards boundary conditions [9] in the case of shear flow or the Kraynik-Reinelt boundary conditions [25] for elongational flow. The basic equations of motion for these algorithms typically exhibit energy growth and need some form of modification if one wants temperature control for the system. Two common choices are the isokinetic Gaussian thermostat and the Nosé-Hoover thermostat. It has been shown that the Nosé-Hoover dynamics is non-ergodic for the NVT ensemble [17,18], and furthermore we are not aware of an analysis of the ergodicity of the Gaussian thermostat for these types of nonequilibrium molecular systems. Instead, we work in a framework that leads to stochastic equations that have a Langevin-type thermostat. In the case of no background flow, the standard Langevin dynamics has long been used to sample the NVT ensemble, and it can be shown to be ergodic.

In the following, we derive a system of equations for a single particle in a microscopic heat bath with a nonzero, constant-in-time background flow in such a way that velocity gradient control and temperature control are incorporated at the same point in the model. The resulting system of equations, which we refer to as the nonequilibrium Langevin dynamics, is

$$
\begin{aligned}
\mathrm{d} \mathbf{Q} & =\mathbf{V} \mathrm{d} t, \\
M \mathrm{~d} \mathbf{V} & =-\gamma(\mathbf{V}-A \mathbf{Q}) \mathrm{d} t+\sigma \mathrm{d} \mathbf{W},
\end{aligned}
$$

where $(\mathbf{Q}, \mathbf{V})$ are the particle's position and velocity, $M$ is the mass of the particle, $\mathbf{W}$ is a standard Brownian motion, $A$ is the homogeneous strain rate, and $\gamma$ and $\sigma$ are scalar constants satisfying the fluctuation-dissipation relation

$$
\gamma=\frac{1}{2} \sigma^{2} \beta
$$

where $\beta=\frac{1}{k_{B} T}$ is the inverse temperature. This system of equations is a generalization of Langevin dynamics, which we recover in the limit $A \rightarrow 0$. In the sequel, we follow the derivation of Dürr, Goldstein, and Lebowitz (DGL) $[6,7]$ who consider the case of a heat bath that has zero background flow.

We briefly summarize the ingredients of the mechanical model used in the derivation. A full description is given in Section 2. The microscopic mechanical model consists of a single large particle immersed in a bath composed of infinitely many small bath atoms. The mass of the large particle $M$ is held constant while the mass of an individual bath atom $m$ is a parameter of the mechanical model. The large particle moves in a ballistic trajectory until it collides with a bath atom, at which point an elastic collision occurs according to (2.7) below, causing a jump in the velocity. The heat bath is constructed in such a way that most of these jumps are independent events distributed according to a velocity measure centered around the background flow. More precisely, the bath atoms have a random initial velocity distribution that is centered around the desired background flow (2.16)-(2.17), with its mean relative kinetic energy proportional to the macroscopic temperature. A microscopic dynamics for the bath atoms is chosen so that the velocity distribution of the bath atoms is preserved (one choice is given by (2.18)), up until they collide with the particle. A typical bath atom has velocity much larger than the velocity of the large particle, and such a bath atom will collide at most once with the large particle (a fact made rigorous in Appendix C). The nonequilibrium Langevin dynamics is then derived as the limiting dynamics of the large particle when $m \rightarrow 0$ (Thm. 2.4).

The full description of the original DGL model as well as two approaches to incorporating background flow are given in Section 2 culminating in the main convergence result, Theorem 2.4. The proof of convergence of the heat bath model to the derived stochastic equations (1.1) is carried out in the Appendices. The proof is structured as in [7], with added arguments to control how the flow in the heat bath affects the error growth. In Section 3 we include numerical results showing the application of the derived equations to computing the shear stress of a Lennard-Jones fluid. 


\section{MODELS FOR NON-UNIFORM BACKGROUND FLOW}

In the following, we consider a system in $\mathbb{R}^{d}$ (for $d=2$ or 3 ) composed of a single, distinguished large particle immersed in a heat bath of light atoms that have mean velocity $A \mathbf{q}$ at point $\mathbf{q}$. We derive the equations of motion of the large particle in the limit as the mass of the individual bath atoms goes to zero. We note that we use the terms "particle" and "atoms" to differentiate the two types of objects, but the heat bath could well be composed of light molecules. We follow the arguments of Dürr, Goldstein, and Lebowitz (DGL) [7], who consider a single large particle placed in an infinite, constant-temperature heat bath with zero background flow (note that the case of a constant uniform background velocity is equivalent to the case of zero background flow by a change of coordinates). The only forces which act on the large particle are due to the heat bath (see Sect. 2.4.3 for extensions to multiple particles and more general interactions). In the limit of small bath atom mass for zero background flow, DGL recover the Langevin dynamics

$$
\begin{aligned}
\mathrm{d} \mathbf{Q} & =\mathbf{V} \mathrm{d} t, \\
M \mathrm{~d} \mathbf{V} & =-\gamma \mathbf{V} \mathrm{d} t+\sigma \mathrm{d} \mathbf{W},
\end{aligned}
$$

where $\mathbf{W}$ denotes a $d$-dimensional Brownian motion, $\mathbf{Q}, \mathbf{V} \in \mathbb{R}^{d}$ denote the position and velocity of the large particle, and $\gamma, \sigma \in \mathbb{R}$ are scalar constants that are determined by the parameters of the large particle and the heat bath and that satisfy the fluctuation-dissipation relation (1.2). Both the drift and diffusion terms in the velocity equation are caused by the elastic collisions of the large particle with the bath atoms, and the randomness stems only from the random initial configuration of the bath. In this paper we extend the work of DGL $[6,7]$ to the case of a nonzero background flow.

In Section 2.1, we review the construction of the heat bath and trajectory of the large particle in the zero-flow case of [7] before outlining in the remaining subsections two possible approaches for extending this to the case of nonzero background flow. The first approach, outlined in Section 2.3, applies to shear flows modeled by a heat bath with one or multiple unidirectional laminar flows. In this approach, the limiting nonequilibrium dynamics is not the dynamics given in (1.1). For reasons outlined in Section 2.3, this first approach is not the one we focus on in this paper. We then describe a second approach in Section 2.4 involving a modified non-Hamiltonian dynamics for the bath atoms, which yields in the limit $m \rightarrow 0$ the nonequilibrium Langevin dynamics (1.1) for general incompressible background flows. Note that, in contrast to the first approach, this second approach is not restricted to shear flows.

In the following, we use bold notation for vectors and normal weight for scalars and matrices. Capital letters are used to distinguish the large particle's position and velocity $(\mathbf{Q}, \mathbf{V})$ from that of a generic bath atom $(\mathbf{q}, \mathbf{v})$.

\subsection{Heat bath with zero background flow}

Here we recall the model and results of [7]. The bath consists of infinitely many light atoms each with position $\mathbf{q} \in \mathbb{R}^{d}$ and velocity $\mathbf{v} \in \mathbb{R}^{d}$. All bath atoms have the same mass, $m>0$, and zero radius. The initial bath configuration is drawn from a Poisson field (whose definition we recall below) with an $m$-dependent measure given by

$$
\mathrm{d} \mu_{m}(\mathbf{v}, \mathbf{q})=\lambda_{m} f_{m}(\mathbf{v}) \mathrm{d} \mathbf{q} \mathrm{d} \mathbf{v}, \quad \mathbf{q}, \mathbf{v} \in \mathbb{R}^{d},
$$

where

$$
\lambda_{m}=m^{-1 / 2} \lambda
$$

is the expected number of atoms per unit volume and $f_{m}$ is the scaled probability distribution on the velocities. The velocity probability distribution scales like

$$
f_{m}(\mathbf{v})=m^{d / 2} f\left(m^{1 / 2} \mathbf{v}\right),
$$

which means that for a single bath atom, the expected speed $\mathbb{E}_{m}(|\mathbf{v}|)=\int_{\mathbb{R}^{d}}|\mathbf{v}| f_{m}(\mathbf{v}) \mathrm{d} \mathbf{v}$ is proportional to $m^{-1 / 2}$. This scaling ensures that the average kinetic energy per atom is constant in the limit $m \rightarrow 0$. The distribution 


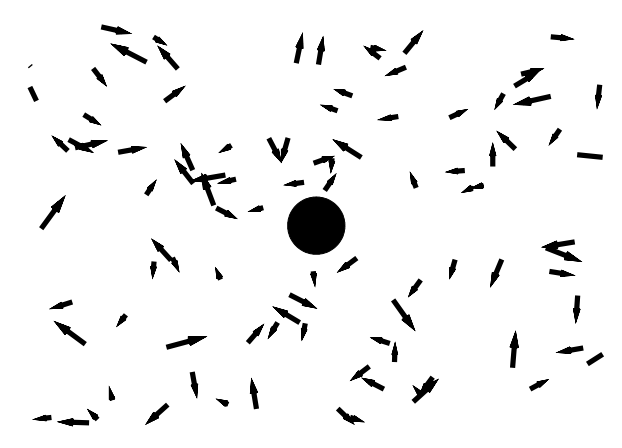

Figure 1. A large particle (of radius $R=2$ ), surrounded by a heat bath with zero background flow, whose atoms are uniformly distributed in space. The velocity of an atom is distributed according to $(2.4)$, with $\beta=1, m=1$, and $\lambda=\frac{1}{16}$.

$f(\mathbf{v})$ is assumed to be rotationally invariant. The quantities

$$
\Phi_{i}=\frac{1}{2} \int_{\mathbb{R}^{d}}\left|v_{1}\right|^{i} f(\mathbf{v}) \mathrm{d} \mathbf{v}, \quad \text { for } i=1, \ldots, 4,
$$

where $v_{1}$ denotes the first component of $\mathbf{v}$, are assumed to be finite. For a set $S \subset \mathbb{R}^{d} \times \mathbb{R}^{d}$ in phase space, with measure denoted by $\mu_{m}(S)$, the number of atoms in $S$, denoted $N_{m}(S)$, is a Poisson random variable with parameter $\mu_{m}(S)$, so that for $k \geq 0$,

$$
\mathbb{P}\left(N_{m}(S)=k\right)=\frac{\mu_{m}(S)^{k}}{k !} \mathrm{e}^{-\mu_{m}(S)} .
$$

Figure 1 shows one possible realization of the heat bath with the choice

$$
f(\mathbf{v})=Z^{-1} \exp \left(-\frac{\beta}{2}|\mathbf{v}|^{2}\right)
$$

where $Z=\left(\frac{2 \pi}{\beta}\right)^{d / 2}$ is the normalization constant.

As soon as the initial condition of the heat bath process has been chosen, the evolution of the system is deterministic. The bath is initialized and a large particle with a finite radius $R$ and mass $M$ is placed in the bath at $(\mathbf{Q}(0), \mathbf{V}(0))$, where we note that the initial condition is independent of $m$. Any bath atoms that are underneath the large particle at the initial time, that is, those such that $|\mathbf{q}(0)-\mathbf{Q}(0)| \leq R$, are removed from the bath (we recall that the bath atoms have zero radius). The bath atoms have no self-interaction and, aside from collisions with the large particle, obey the dynamics

$$
\begin{aligned}
\mathrm{d} \mathbf{q} & =\mathbf{v} \mathrm{d} t \\
\mathrm{~d} \mathbf{v} & =0
\end{aligned}
$$

Likewise, the large particle's position and velocity evolve according to

$$
\begin{aligned}
\mathrm{d} \mathbf{Q}_{m} & =\mathbf{V}_{m} \mathrm{~d} t, \\
\mathrm{~d} \mathbf{V}_{m} & =0 .
\end{aligned}
$$

We explicitly denote the $m$-dependence of the mechanical process, while for notational convenience, we omit the $m$-dependence of the bath coordinates. 
When a bath atom encounters the large particle, it undergoes an elastic collision. For a single collision, let $\mathbf{e}_{\mathrm{n}}$ denote the unit vector in the direction from the bath atom to the particle center. Let $\mathbf{V}_{\mathrm{n}}=\left(\mathbf{V} \cdot \mathbf{e}_{\mathrm{n}}\right) \mathbf{e}_{\mathrm{n}}$ and $\mathbf{V}_{\mathrm{t}}=\mathbf{V}-\mathbf{V}_{\mathrm{n}}$ denote the velocity of the large particle in the normal and tangential directions. Similarly, define $\mathbf{v}_{\mathrm{n}}=\left(\mathbf{v} \cdot \mathbf{e}_{\mathrm{n}}\right) \mathbf{e}_{\mathrm{n}}$ and $\mathbf{v}_{\mathrm{t}}=\mathbf{v}-\mathbf{v}_{\mathrm{n}}$ for the bath atoms. We distinguish between the component of $\mathbf{V}$ in the normal direction $\mathbf{V}_{\mathrm{n}}=\left(\mathbf{V} \cdot \mathbf{e}_{\mathrm{n}}\right) \mathbf{e}_{\mathrm{n}}$ and its magnitude $V_{\mathrm{n}}=\mathbf{V} \cdot \mathbf{e}_{\mathrm{n}}$. The elastic collision rule is

$$
\begin{aligned}
\mathbf{V}_{\mathrm{t}}^{\prime} & =\mathbf{V}_{\mathrm{t}}, \quad \mathbf{v}_{\mathrm{t}}^{\prime}=\mathbf{v}_{\mathrm{t}}, \\
\mathbf{V}_{\mathrm{n}}^{\prime} & =\frac{M-m}{M+m} \mathbf{V}_{\mathrm{n}}+\frac{2 m}{M+m} \mathbf{v}_{\mathrm{n}}, \\
\mathbf{v}_{\mathrm{n}}^{\prime} & =-\frac{M-m}{M+m} \mathbf{v}_{\mathrm{n}}+\frac{2 M}{M+m} \mathbf{V}_{\mathrm{n}},
\end{aligned}
$$

where primes denote the after-collision velocities. The generated trajectory $\left(\mathbf{Q}_{m}, \mathbf{V}_{m}\right)$ is called the mechanical process. It is possible to only consider the case of single collisions since it has been shown that pathologies such as multiple simultaneous collisions or an infinite number of collisions in a finite time interval have zero probability (see Appendix of [7] and also Appendix E). As a result of the above, the mechanical process is well-defined on any finite time interval.

Each initial condition of the heat bath corresponds to a realization of the mechanical process $\left(\mathbf{Q}_{m}, \mathbf{V}_{m}\right)$. The velocity $\mathbf{V}_{m}$ is defined to have right-continuous jumps so that the trajectory is a càdlàg function, that is, a piecewise continuous function that is everywhere right-continuous and has a left-hand limit. For a fixed initial condition of the heat bath, the particle trajectory $\left(\mathbf{Q}_{m}, \mathbf{V}_{m}\right)$ is a deterministic process; however, since the bath's initial condition is a random variable, the particle's position and velocity are also random variables.

Remark 2.1. The trajectory $\left(\mathbf{Q}_{m}, \mathbf{V}_{m}\right)$ is not a Markov process. It would only be Markov if the rate of collisions with bath atoms did not depend on the history of the particle; however, this is not the case because of two effects. First, recollisions are possible with bath atoms that are moving sufficiently slowly. Second, certain collisions, called "virtual collisions", with slow-moving atoms are made impossible due to the particle "sweeping out" a path behind it. The large particle creates a wake behind itself, and whenever it decelerates, there is a minimum delay before slow moving atoms can hit it from behind. To show convergence of the mechanical process to the limiting Langevin dynamics (2.1), one must show that these history effects are negligible in the limit $m \rightarrow 0$.

We now define the appropriate topology in order to precisely state the convergence result of [7]. We fix a finite time $T$ and let $\mathcal{D}([0, T])$ denote the space of càdlàg functions on the interval $[0, T]$. For functions $f, g \in \mathcal{D}([0, T])$, we define the Skorokhod metric (see for example [2])

$$
\sigma_{\mathrm{sk}}(f, g)=\inf _{\lambda \in \Lambda} \max \left\{\|\lambda-\operatorname{Id}\|_{L^{\infty}([0, T])},\|f-g \circ \lambda\|_{L^{\infty}\left([0, T] ; \mathbb{R}^{d}\right)}\right\},
$$

where Id : $[0, T] \rightarrow[0, T]$ denotes the identity map and $\Lambda$ is the set of all strictly increasing, continuous bijections of $[0, T]$. For vector-valued $f$, we apply the Euclidean norm in space so that the $L^{\infty}$-norm above is given by

$$
\|f\|_{L^{\infty}\left([0, T] ; \mathbb{R}^{d}\right)}=\operatorname{ess~sup}_{t \in[0, T]}|f(t)| .
$$

The mechanical process $\left(\mathbf{Q}_{m}, \mathbf{V}_{m}\right)$ defined above is in $\mathcal{D}([0, T])$.

We now recall the following definitions for the convergence of random variables.

Definition 2.2. Let $Z_{m}$ and $Z$ be random variables in the metric space $\left(\mathcal{D}([0, T]), \sigma_{\mathrm{sk}}\right)$. We denote convergence in law by $\left(Z_{m}\right)_{\{0 \leq t \leq T\}} \stackrel{\mathcal{L}}{\longrightarrow} Z_{\{0 \leq t \leq T\}}$, that is, $\mathbb{E} f\left(Z_{m}\right) \rightarrow \mathbb{E} f(Z)$ as $m \rightarrow 0$ for all bounded, continuous functions $f: \mathcal{D}([0, T]) \rightarrow \mathbb{R}$. We denote convergence in probability by $\left(Z_{m}\right)_{\{0 \leq t \leq T\}} \stackrel{p}{\longrightarrow}(Z)_{\{0 \leq t \leq T\}}$, that is, for all $\varepsilon>0$,

$$
\lim _{m \rightarrow 0} \mathbb{P}\left(\left\{\sigma_{\mathrm{sk}}\left(Z_{m}, Z\right)>\varepsilon\right\}\right)=0 .
$$


The following theorem gives not only convergence in law of the mechanical process to the Langevin dynamics (2.1), but also an explicit expression for the coefficients of the limiting dynamics:

$$
\gamma=\frac{4 \lambda R^{d-1} S_{d-1}}{d} \Phi_{1}, \quad \sigma=\left[\frac{4 \lambda R^{d-1} S_{d-1}}{d} \Phi_{3}\right]^{\frac{1}{2}},
$$

where $S_{d-1}$ is the surface area of the $(d-1)$-sphere $\mathbb{S}^{d-1}, \Phi_{i}$ is defined in (2.3), $R$ and $M$ are the radius and mass of the large particle, and $\lambda$ is a density parameter of the bath in (2.2).

Theorem 2.3 ([7], Thm. 2.1). Consider the mechanical process $\left(\mathbf{Q}_{m}, \mathbf{V}_{m}\right)$, defined in (2.5), (2.6), and (2.7) with bath measure defined in $(2.2)$, and let $(\mathbf{Q}, \mathbf{V})$ denote the solution to the Langevin equation (2.1) with coefficients (2.9). For any $T>0$,

$$
\left(\mathbf{Q}_{m}, \mathbf{V}_{m}\right)_{\{0 \leq t \leq T\}} \stackrel{\mathcal{L}}{\longrightarrow}(\mathbf{Q}, \mathbf{V})_{\{0 \leq t \leq T\}}
$$

as $m \rightarrow 0$, where convergence is with respect to the Skorokhod metric $(2.8)$ on $\mathcal{D}([0, T])$.

The resulting dynamics (2.1) satisfies the fluctuation-dissipation relation (1.2) provided that

$$
\frac{\Phi_{1}}{\Phi_{3}}=\frac{\beta}{2},
$$

which holds true for $f(\mathbf{v})$ as in (2.4), for example. In this case, the temperature of the large particle is identical to that of the bath. The main result of this paper, Theorem 2.4, is the generalization of Theorem 2.3 to the case where the heat bath has a nonzero background flow.

\subsection{Generalization to a nonzero background flow}

We generalize the model described above by considering a bath measure $\mathrm{d} \mu_{m}(\mathbf{q}, \mathbf{v})$ where, in contrast to the previous section, the initial distribution of the velocities depends on the position. We give two possible approaches to the generalization. In the first, which applies only to shear flow, the velocities are restricted to laminar profiles. For the second, which applies to general incompressible flows, we change the microscopic dynamics that the bath atoms satisfy in order to preserve the distribution of atoms. In both cases, we require that the bath measure and dynamics satisfy the following hypotheses:

The average velocity at point $\mathbf{q}^{*}$ is equal to $A \mathbf{q}^{*}$ in the sense that

$$
\text { for all } \mathbf{q}^{*} \in \mathbb{R}^{d}, \lim _{\varepsilon \rightarrow 0} \frac{\int_{\mathbb{R}^{d}} \int_{\mathcal{B}\left(\mathbf{q}^{*}, \varepsilon\right)} \mathbf{v} \mathrm{d} \mu_{m}(\mathbf{q}, \mathbf{v})}{\int_{\mathbb{R}^{d}} \int_{\mathcal{B}\left(\mathbf{q}^{*}, \varepsilon\right)} \mathrm{d} \mu_{m}(\mathbf{q}, \mathbf{v})} \text { exists and is equal to } A \mathbf{q}^{*},
$$

The measure $\mathrm{d} \mu_{m}(\mathbf{q}, \mathbf{v})$ is invariant under the bath dynamics in the absence of collisions,

where $\mathcal{B}(\mathbf{q}, \varepsilon)$ denotes the ball of radius $\varepsilon$ about $\mathbf{q}$. The invariance of the bath measure means that, except for the effects described in Remark 2.1, the large particle experiences collisions with a time-independent rate. We note that satisfying (H1) and (H2) is not trivial. For example, if the velocities $\mathbf{v}$ have a Gaussian distribution centered around $A \mathbf{q}$ and the atoms follow ballistic trajectories (2.5), then the system satisfies (H1) but not (H2). In both generalizations described below, the bath atoms interact with the large particle only through elastic collisions as in (2.7).

In Section 2.3, we describe a laminar flow model for the specific case where the background flow is a shear. All bath atoms are restricted to move in one of the coordinate directions, following otherwise ballistic trajectories. The resulting dynamics is not the same as the nonequilibrium Langevin dynamics (1.1) that form the main 


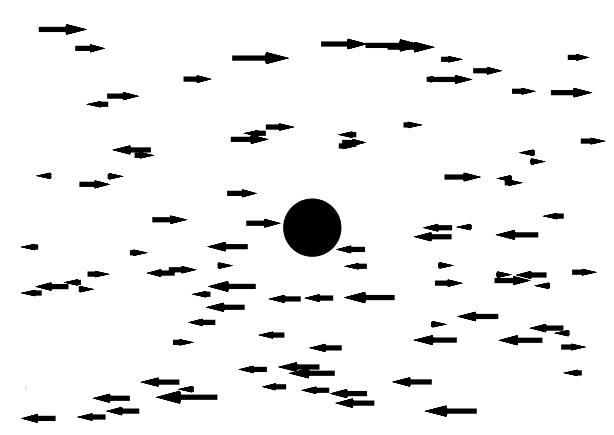

Figure 2. A large particle (of radius $R=2$ ) surrounded by a laminar heat bath whose atoms are distributed according to (2.11). The velocity is centered around the constant shear profile $A \mathbf{q}$, where $A$ is given in (2.10). Since the bath atoms may only flow parallel to the shear, the variance around the mean velocity along each flow line $q_{2}=c$ is constant for all time. We have chosen $s=0.1, \beta=1, m=1$, and $\lambda=\frac{1}{16}$.

focus of this work. In Section 2.4 we do not restrict the velocity directions, and we choose a modified (nonHamiltonian) dynamics for the bath atoms which leaves the chosen bath measure invariant for any traceless $A$. This approach leads to the nonequilibrium Langevin dynamics (1.1) as limiting equations for the large particle in the limit $m \rightarrow 0$.

\subsection{Laminar flow models}

For this subsection, we restrict ourselves to a heat bath in $\mathbb{R}^{3}$ under shear flow with the specific strain rate

$$
A=\left[\begin{array}{lll}
0 & s & 0 \\
0 & 0 & 0 \\
0 & 0 & 0
\end{array}\right],
$$

for some given $s \in \mathbb{R}$. We describe two variants of a laminar flow model, which both create a background shear flow by restricting the atom velocities to be parallel to the coordinate axes.

\subsubsection{Single laminar flow}

We enforce a steady shear flow for the bath atoms by choosing initial velocities that are nonzero only in the first coordinate, $\mathbf{e}_{1}=[1,0,0]^{T}$. We choose the initial configuration of the bath atoms according to a Poisson field with measure

$$
\mathrm{d} \mu_{m}(\mathbf{q}, \mathbf{v})=\lambda_{m} Z^{-1} m^{1 / 2} \exp \left(-\frac{\beta}{2} m\left(v_{1}-s q_{2}\right)^{2}\right) \delta\left(v_{2}\right) \delta\left(v_{3}\right) \mathrm{d} \mathbf{q} \mathrm{d} \mathbf{v},
$$

where $Z=\left(\frac{2 \pi}{\beta}\right)^{1 / 2}$ and $\delta$ denotes the Dirac distribution (see Fig. 2 for an example initial condition). The bath atoms follow ballistic motion (2.5), and it is easily verified that the bath satisfies (H1) and (H2). The bath atoms undergo elastic collisions (2.7) with the large particle.

Ignoring the effect of recollisions, we formally compute the limiting dynamics as $m \rightarrow 0$ to be

$$
\begin{aligned}
\mathrm{d} \mathbf{Q} & =\mathbf{V} \mathrm{d} t, \\
M \mathrm{~d} \mathbf{V} & =-\gamma(\mathbf{V}-A \mathbf{Q}) \mathrm{d} t+\sigma \mathrm{d} \mathbf{W},
\end{aligned}
$$


where

$$
\gamma=\frac{2 \sqrt{2 \pi} \lambda R^{2}}{\sqrt{\beta}}\left(\begin{array}{ccc}
1 & 0 & 0 \\
0 & \frac{1}{2} & 0 \\
0 & 0 & \frac{1}{2}
\end{array}\right), \quad \sigma=\left[\frac{4 \sqrt{2 \pi} \lambda R^{2}}{\sqrt{\beta^{3}}}\left(\begin{array}{ccc}
\frac{2}{3} & 0 & 0 \\
0 & \frac{1}{6} & 0 \\
0 & 0 & \frac{1}{6}
\end{array}\right)\right]^{\frac{1}{2}} .
$$

The details of this computation are given in Appendix F. We note that for the laminar flow model considered here we have not rigorously proven a convergence result and, in particular, we have not justified ignoring recollisions. On the other hand, a proof is carried out for the model presented in Section 2.4.

The resulting dissipation and noise terms are both anisotropic, which is due to the fact that the background laminar flow is itself anisotropic. These terms are larger in the direction of the flow, and the dynamics do not satisfy a standard fluctuation-dissipation relation in which $\frac{\beta}{2} \gamma^{-1} \sigma \sigma^{T}=1$, since

$$
\frac{\beta}{2} \gamma^{-1} \sigma \sigma^{T}=\left(\begin{array}{ccc}
\frac{2}{3} & 0 & 0 \\
0 & \frac{1}{3} & 0 \\
0 & 0 & \frac{1}{3}
\end{array}\right)
$$

Furthermore, in the case where the shear flow is identically zero, $A=0$, the dynamics does not reduce to the standard Langevin dynamics (2.1) which was derived for the case of zero background flow. Since we desire a system of equations that reduces to Langevin dynamics in the case $A=0$ (in particular because the Langevin dynamics is a widely accepted dynamics to model fluid flows at equilibrium), we consider a modification for removing the anisotropy.

\subsubsection{Multiple laminar flows}

One may attempt to create an isotropic flow by overlaying multiple background flows. We consider the superposition of three distinct laminar flows, each one with all velocities aligned in a single direction, $\mathbf{e}_{i}$. Then we choose the initial bath coordinates according to a Poisson field with distribution function

$$
\begin{aligned}
\mathrm{d} \mu_{m}(\mathbf{q}, \mathbf{v})= & {\left[\frac{1}{3} \lambda_{m} Z^{-1} m^{1 / 2} \exp \left(-\frac{\beta}{2} m\left(v_{1}-s q_{2}\right)^{2}\right) \delta\left(v_{2}\right) \delta\left(v_{3}\right),\right.} \\
& +\frac{1}{3} \lambda_{m} Z^{-1} m^{1 / 2} \delta\left(v_{1}\right) \exp \left(-\frac{\beta}{2} m v_{2}^{2}\right) \delta\left(v_{3}\right) \\
& \left.+\frac{1}{3} \lambda_{m} Z^{-1} m^{1 / 2} \delta\left(v_{1}\right) \delta\left(v_{2}\right) \exp \left(-\frac{\beta}{2} m v_{3}^{2}\right)\right] \mathrm{d} \mathbf{q} \mathrm{d} \mathbf{v} .
\end{aligned}
$$

Each atom moves in one of the three coordinate directions, since the presence of the delta distributions means that at most one of the velocity coordinates is nonzero. Since the bath atoms do not have any self-interaction, the different flows do not "mix" in any way, and in particular this bath measure is invariant under ballistic motion. Figure 3 depicts a realization of this bath in the two-dimensional case.

According to the formal calculations carried out in Appendix F, where we again ignore the effect of recollisions, we find that the limiting dynamics of the large particle is

$$
\begin{aligned}
\mathrm{d} \mathbf{Q} & =\mathbf{V} \mathrm{d} t \\
M \mathrm{~d} \mathbf{V} & =-\gamma\left(\mathbf{V}-\frac{1}{2} A \mathbf{Q}\right) \mathrm{d} t+\sigma \mathrm{d} \mathbf{W},
\end{aligned}
$$

where

$$
\gamma=\frac{4 \sqrt{2 \pi} \lambda R^{2}}{\sqrt{\beta}}, \quad \sigma=\left[\frac{4 \sqrt{2 \pi} \lambda R^{2}}{\sqrt{\beta^{3}}}\right]^{1 / 2} .
$$

As desired, the dynamics in (2.15) is no longer an anisotropic equation in contrast to (2.12) since $\gamma$ and $\sigma$ are scalar quantities. We note that the damping term on the large particle is relative to half the velocity of the 

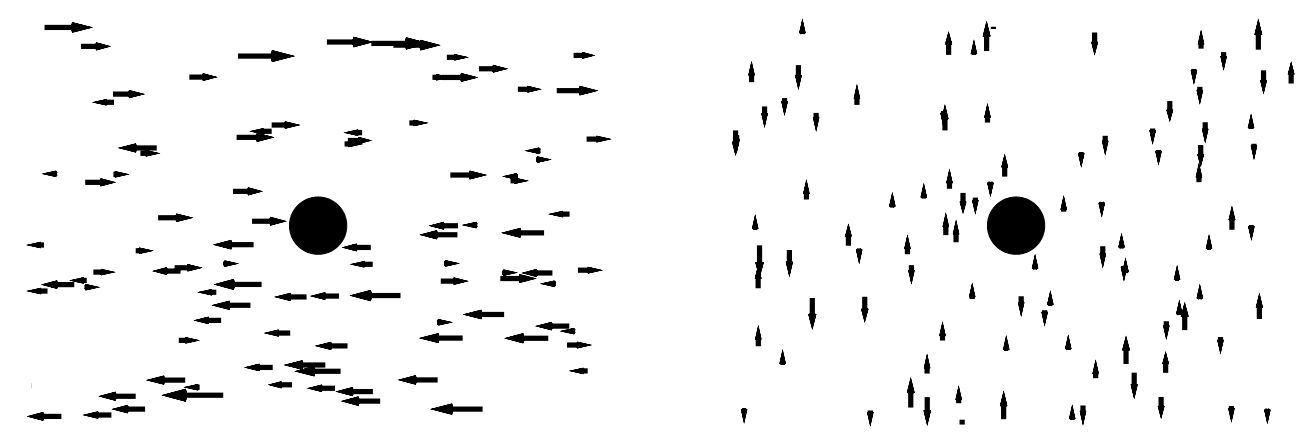

Figure 3. Two components of the laminar bath which are superimposed to create an environment for the particle consistent with the measure (2.14). We have chosen $s=0.1, \beta=1$, $m=1$, and $\lambda=\frac{1}{16}$.

background shear flow and that the fluctuation-dissipation relation is not satisfied, since $\frac{\beta}{2} \gamma^{-1} \sigma^{2}=\frac{1}{2} \neq 1$. While we have chosen the inverse temperature for each bath equal to $\beta$ in equation (2.14), we find in Appendix $\mathrm{F}$ that there is no choice of inverse temperatures for the three laminar bath components that allows us to construct an isotropic damping in which the damping term is relative to the full background flow. We note that this issue is not specific to the three dimensional case considered here.

We do not pursue this model further for two reasons. First and foremost, it is not clear how to generalize these laminar bath flow models from simple shear flows to more general incompressible flows. Second, a heat bath with temperature proportional to $\beta^{-1}$ and strain rate $A$ gives a macroscopic dynamics whose temperature is proportional to $\frac{1}{2} \beta^{-1}$ and whose damping is relative to $\frac{1}{2} A$. We expect damping relative to $A$ as in (1.1), which agrees with NEMD dynamics such as g-SLLOD (see Sect. 2.4.2 for a description of the g-SLLOD dynamics and their relation to (1.1)). While it would be possible to choose bath parameters $A_{\text {bath }}=2 A$ and $\beta_{\text {bath }}=\frac{1}{2} \beta$ to give the desired macroscopic parameters, $A$ and $\beta$, to the large particle, we instead consider a different approach which both handles general incompressible flows and shows agreement between the bath parameters and the parameters of the derived dynamics.

\subsection{Non-Hamiltonian bath dynamics}

In this section we describe an approach based on modifying the bath dynamics to no longer follow ballistic trajectories (2.5). The particle velocity accelerates so that the distribution of velocities relative to the background flow is preserved.

\subsubsection{Model and convergence results}

We consider a bath whose initial condition is characterized by the measure

$$
\mathrm{d} \mu_{m}(\mathbf{q}, \mathbf{v})=\lambda_{m} f_{m}(\mathbf{q}, \mathbf{v}) \mathrm{d} \mathbf{q} \mathrm{d} \mathbf{v} \quad \text { for } \mathbf{q}, \mathbf{v} \in \mathbb{R}^{d},
$$

where $f_{m}$ has the form

$$
f_{m}(\mathbf{q}, \mathbf{v})=m^{d / 2} f\left(m^{1 / 2}(\mathbf{v}-A \mathbf{q})\right)
$$

for $A \in \mathbb{R}^{d \times d}$ (Fig. 4) and where $\lambda_{m}=m^{-1 / 2} \lambda$. As in Section 2.1, we assume that $f(\mathbf{v})$ is a rotationally invariant probability distribution, and that the first four moments are finite. Any distribution of the form (2.16) with (2.17) satisfies (H1). We additionally assume that $f$ is a decreasing function of $|\mathbf{v}|$. This additional assumption is used below when approximating the trajectory of the mechanical process with a Markov process in Appendix D. 


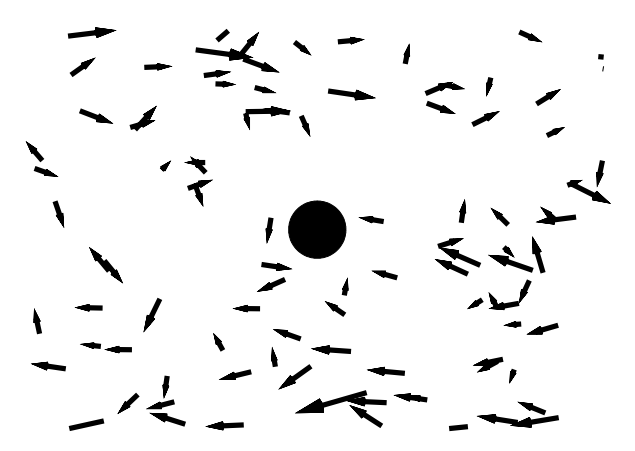

Figure 4. A large particle (of radius $R=2$ ) immersed in a heat bath whose atoms are distributed according to (2.17). The velocities have Gaussian distribution centered around a shear flow, with $A$ given in (2.10). We have chosen $s=0.1, \beta=1, m=1$, and $\lambda=\frac{1}{16}$.

In order to satisfy (H2), we now change the underlying bath dynamics and consider the following nonHamiltonian dynamics for the bath atoms:

$$
\begin{aligned}
& \mathrm{d} \mathbf{q}=\mathbf{v} \mathrm{d} t \\
& \mathrm{~d} \mathbf{v}=A \mathbf{v} \mathrm{d} t
\end{aligned}
$$

It is still the case that bath atoms do not interact with one another, but now the bath atoms accelerate in a way that leaves the desired velocity distribution (2.16)-(2.17) invariant. The corresponding Liouville equation is

$$
\partial_{t} \rho(\mathbf{q}, \mathbf{v}, t)=-\nabla_{\mathbf{q}} \cdot(\mathbf{v} \rho(\mathbf{q}, \mathbf{v}, t))-\nabla_{\mathbf{v}} \cdot(A \mathbf{v} \rho(\mathbf{q}, \mathbf{v}, t))
$$

As long as $\operatorname{tr} A=0$, which is equivalent to having an incompressible background flow, any function of the form $\rho(\mathbf{q}, \mathbf{v}, t)=f(\mathbf{v}-A \mathbf{q})$ is invariant under the dynamics. This invariance is our motivation for choosing the dynamics (2.18), and in particular, we are not restricted to shear flows. We interpret the dynamics by considering the relative velocity

$$
\overline{\mathbf{v}}=\mathbf{v}-A \mathbf{q}
$$

In terms of $(\mathbf{q}, \overline{\mathbf{v}})$, the dynamics $(2.18)$ is

$$
\begin{aligned}
\mathrm{d} \mathbf{q} & =(A \mathbf{q}+\overline{\mathbf{v}}) \mathrm{d} t \\
\mathrm{~d} \overline{\mathbf{v}} & =0 .
\end{aligned}
$$

Thus the relative velocity, $\overline{\mathbf{v}}$, does not change in time and the velocity of the atoms is at all times equal to the background flow plus an initial perturbation of thermal origin. However, the choice of dynamics (2.18) is motivated by the fact that it satisfies (H2), rather than by the above physical interpretation.

As before, the large particle undergoes elastic collisions with the bath atoms according to the rule (2.7). In the limit $m \rightarrow 0$, the particle dynamics converges to the nonequilibrium Langevin dynamics (1.1) which we recall here:

$$
\begin{aligned}
\mathrm{d} \mathbf{Q} & =\mathbf{V} \mathrm{d} t, \\
M \mathrm{~d} \mathbf{V} & =-\gamma(\mathbf{V}-A \mathbf{Q}) \mathrm{d} t+\sigma \mathrm{d} \mathbf{W},
\end{aligned}
$$


where, as in the zero background flow case (2.9),

$$
\gamma=\frac{4 \lambda R^{d-1} S_{d-1}}{d} \Phi_{1}, \quad \sigma=\left[\frac{4 \lambda R^{d-1} S_{d-1}}{d} \Phi_{3}\right]^{\frac{1}{2}},
$$

where $S_{d-1}$ denotes the surface area of the $(d-1)$-sphere, and $\Phi_{i}$ is defined in (2.3). As in the case of zero background flow in Section 2.1, this dynamics satisfies a standard fluctuation-dissipation relation (1.2), with temperature equal to the bath's temperature, provided that

$$
\frac{\Phi_{1}}{\Phi_{3}}=\frac{\beta}{2},
$$

which holds true for the Gaussian distribution $f(\mathbf{v})=Z^{-1} \exp \left(-\frac{\beta}{2}|\mathbf{v}|^{2}\right)$, for example. We establish the following result in the Appendices A-E.

Theorem 2.4. Consider the mechanical process $\left(\mathbf{Q}_{m}, \mathbf{V}_{m}\right)$, defined in (2.6), (2.7), and (2.18) with bath measure defined in (2.16) and (2.17), and let $(\mathbf{Q}, \mathbf{V})$ denote the solution to the nonequilibrium Langevin equations (2.21) with coefficients (2.22). For any $T>0$,

$$
\left(\mathbf{Q}_{m}, \mathbf{V}_{m}\right)_{\{0 \leq t \leq T\}} \stackrel{\mathcal{L}}{\longrightarrow}(\mathbf{Q}, \mathbf{V})_{\{0 \leq t \leq T\}}
$$

as $m \rightarrow 0$, where convergence is with respect to the Skorokhod metric $(2.8)$ on $\mathcal{D}([0, T])$.

The structure of the proof is the same as in [7]: we use a Markov approximation to the mechanical process and split the convergence proof into two steps. In Appendix A, we define the Markov process that approximates the mechanical process and outline in more detail the convergence proof. In Appendix B, we show that the Markov process converges in law to the solution of the nonequilibrium Langevin dynamics (2.21). In Appendices C and D we show that the difference between the Markov process and the mechanical process converges in probability to zero. These two convergence results are enough to deduce that the mechanical process converges in law to the nonequilibrium Langevin dynamics [2]. In Appendix E, we show that the mechanical process is well-defined for almost every initial condition up to a positive stopping time.

\subsubsection{Relationship to $g$-SLLOD}

The g-SLLOD equations of motion [9,27] (also called p-SLLOD [8]) are given by

$$
\begin{aligned}
\mathrm{d} \mathbf{Q}_{i} & =\left(A \mathbf{Q}_{i}+\overline{\mathbf{V}}_{i}\right) \mathrm{d} t, \\
M \mathrm{~d} \overline{\mathbf{V}}_{i} & =\left(-\nabla_{\mathbf{Q}_{i}} E(\mathbf{Q})-M A \overline{\mathbf{V}}_{i}-M A A \mathbf{Q}_{i}\right) \mathrm{d} t .
\end{aligned}
$$

Note that these equations are written in terms of relative velocity, $\overline{\mathbf{V}}_{i}=\mathbf{V}_{i}-A \mathbf{Q}_{i}$. This system of equations is used to simulate a molecular system in a non-zero background flow. The particles interact through the potential field $E(\mathbf{Q})$. The g-SLLOD equations are simply a change of variables from the Newton equations of motion in the reference frame

$$
\begin{aligned}
\mathrm{d} \mathbf{Q}_{i} & =\mathbf{V}_{i} \mathrm{~d} t, \\
M \mathrm{~d} \mathbf{V}_{i} & =-\nabla_{\mathbf{Q}_{i}} E(\mathbf{Q}) \mathrm{d} t,
\end{aligned}
$$

to the relative velocity. If we add a Langevin damping term and noise term to the dynamics in (2.23), we have

$$
\begin{aligned}
\mathrm{d} \mathbf{Q}_{i} & =\left(\overline{\mathbf{V}}_{i}+A \mathbf{Q}_{i}\right) \mathrm{d} t, \\
M \mathrm{~d} \overline{\mathbf{V}}_{i} & =\left(-\nabla_{\mathbf{Q}_{i}} E(\mathbf{Q})-M A \overline{\mathbf{V}}_{i}-M A A \mathbf{Q}_{i}\right) \mathrm{d} t-\gamma \overline{\mathbf{V}}_{i} \mathrm{~d} t+\sigma \mathrm{d} \mathbf{W} .
\end{aligned}
$$


Transforming back to the reference frame, this gives

$$
\begin{aligned}
\mathrm{d} \mathbf{Q}_{i} & =\mathbf{V}_{i} \mathrm{~d} t, \\
M \mathrm{~d} \mathbf{V}_{i} & =-\nabla_{\mathbf{Q}_{i}} E(\mathbf{Q}) \mathrm{d} t-\gamma\left(\mathbf{V}_{i}-A \mathbf{Q}_{i}\right) \mathrm{d} t+\sigma \mathrm{d} \mathbf{W},
\end{aligned}
$$

which is (2.21), with the addition of an external potential $E$. Thus, our construction is consistent with the application of a Langevin thermostat to the g-SLLOD equations of motion and thus provides a derivation of the g-SLLOD equations from a heat bath model.

\subsubsection{Generalizations}

We have chosen the microscopic dynamics (2.6) for the large particle and (2.18) for the bath atoms. First, we may make a more general choice of microscopic dynamics for the large particle between collisions,

$$
\begin{aligned}
\mathrm{d} \mathbf{Q}_{m} & =\mathbf{V}_{m} \mathrm{~d} t, \\
M \mathrm{~d} \mathbf{V}_{m} & =F\left(\mathbf{Q}_{m}, \mathbf{V}_{m}\right) \mathrm{d} t,
\end{aligned}
$$

replacing (2.6) with (2.25) in the definition of the mechanical process. We assume $F$ is uniformly Lipschitz continuous on $\mathbb{R}^{d} \times \mathbb{R}^{d}$ and $\nabla_{\mathbf{V}} \cdot F(\mathbf{Q}, \mathbf{V})=0$ which implies that both the mechanical process for finite $m$ and the limiting SDE are well-posed (the proof given in Appendix $\mathrm{E}$ for the $F=0$ case depends on the fact that the flow map of the mechanical process preserves Lebesgue measure). The limiting dynamics of the large particle becomes

$$
\begin{aligned}
\mathrm{d} \mathbf{Q} & =\mathbf{V} \mathrm{d} t, \\
M \mathrm{~d} \mathbf{V} & =F(\mathbf{Q}, \mathbf{V}) \mathrm{d} t-\gamma(\mathbf{V}-A \mathbf{Q}) \mathrm{d} t+\sigma \mathrm{d} \mathbf{W} .
\end{aligned}
$$

The proof of this result is a straightforward extension of the analysis given here, where the Lipschitz continuity allows us to bound the particle trajectory over finite time intervals.

For the specific case of $F(\mathbf{Q}, \mathbf{V})=M A \mathbf{V}$ with $\operatorname{tr} A=0$, which is the same acceleration term as in the microscopic dynamics chosen for the bath, we find the stochastic dynamics

$$
\begin{aligned}
\mathrm{d} \mathbf{Q} & =\mathbf{V} \mathrm{d} t \\
M \mathrm{~d} \mathbf{V} & =M A \mathbf{V} \mathrm{d} t-\gamma(\mathbf{V}-A \mathbf{Q}) \mathrm{d} t+\sigma \mathrm{d} \mathbf{W} .
\end{aligned}
$$

This dynamics was obtained in [19] for the case of shear flow by applying the Kac-Zwanzig formalism, thereby obtaining a generalized Langevin dynamics, and assuming that the memory kernel converges to a delta function (that is, there is no memory in the system). The dynamics (2.26) is of particular interest since we can explicitly check that $\rho(\mathbf{Q}, \mathbf{V})=\exp \left(-\frac{\beta}{2}|\mathbf{V}-A \mathbf{Q}|^{2}\right)$ is an invariant measure of (2.26). In contrast we do not know an analytical expression for stationary states of (2.21) for general choice of $A$.

Second, we could consider the case of a non-homogeneous incompressible background flow, where the average velocity at the point $\mathbf{q}^{*}$ in (H1) is $\mathbf{u}\left(\mathbf{q}^{*}\right)$ where $\mathbf{u}$ is a divergence-free vector field. For any such $\mathbf{u}$, the microscopic bath dynamics

$$
\begin{aligned}
\mathrm{d} \mathbf{q} & =\mathbf{v} \mathrm{d} t \\
\mathrm{~d} \mathbf{v} & =\nabla \mathbf{u}(\mathbf{q}) \mathbf{v} \mathrm{d} t
\end{aligned}
$$

preserves any bath measure of the form (2.16)-(2.17) with $\mathbf{v}-A \mathbf{q}$ replaced by $\mathbf{v}-\mathbf{u}(\mathbf{q})$. In this case, the dynamics of the large particle in the mechanical process converges, in the limit $m \rightarrow 0$, to

$$
\begin{aligned}
\mathrm{d} \mathbf{Q} & =\mathbf{V} \mathrm{d} t \\
M \mathrm{~d} \mathbf{V} & =-\gamma(\mathbf{V}-\mathbf{u}(\mathbf{Q})) \mathrm{d} t+\sigma \mathrm{d} \mathbf{W} .
\end{aligned}
$$


This extension likewise does not pose any theoretical difficulties, at least when $\mathbf{u}$ is Lipschitz continuous. This reduces to the case presented above when $\mathbf{u}(\mathbf{q})=A \mathbf{q}$, where $\operatorname{div} \mathbf{u}(\mathbf{q})=0$ is equivalent to $\operatorname{tr} A=0$.

Finally, a much more challenging extension is the case of multiple large particles. The restriction to a single large particle is a necessary assumption for the argument here and in $[3,6,7]$, since it allows us to estimate the distribution of velocities of the bath atoms that collide with the particle. In particular, fast moving bath atoms can collide at most once with the large particle, whereas in the multi-particle case these atoms could bounce between large particles and possibly collide with them many times. Extending the proof of Theorems 2.3 and 2.4 to the case of multiple particles immersed in a bath is a non-trivial task, as atoms of any speed can bounce between the large particles, and there is also a shadowing effect that particles have on one another. Kotelenez [13] treated the multi-particle case using a mean field interaction representing a mesoscale interaction and showed how the bath can generate close-range forces among the large particles. Kusuoka and Liang treated the multi-particle case as well, using a weaker bath-particle interaction which prevents atoms from bouncing back and forth among the large particles [15]. We do not pursue a multi-particle derivation in this work; however, we do perform numerical tests of a natural extension of the limiting equations (2.21) to the multi-particle case in Section 3.

\section{Numerics FOR THE MULTi-PARTICLE CASE}

We consider the application of the derived equations (2.21) to the simulation of a fluid composed of many identical large particles. We note that the derivation for Theorem 2.4 only applies to the case of a single particle, with no forcing except through the bath, whereas we now apply the equation to a system with many large particles that interact with one another. The many-particle case is the case of interest in applications, and we numerically investigate the qualitative behavior of the proposed dynamics to test its suitability for sampling flows with a given mean behavior. We consider a system in $\mathbb{R}^{3}$ consisting of $N$ large particles whose position and velocity $(\mathbf{Q}, \mathbf{V}) \in \mathbb{R}^{6 N}$ evolve according to the dynamics (2.24) which we recall here:

$$
\begin{aligned}
\mathrm{d} \mathbf{Q}_{i} & =\mathbf{V}_{i} \mathrm{~d} t, \\
M \mathrm{~d} \mathbf{V}_{i} & =-\nabla_{\mathbf{Q}_{i}} E(\mathbf{Q}) \mathrm{d} t-\gamma\left(\mathbf{V}_{i}-A \mathbf{Q}_{i}\right) \mathrm{d} t+\sigma \mathrm{d} \mathbf{W}_{i} .
\end{aligned}
$$

The index $i=1, \ldots, N$ runs over all particles, whereas the lack of index in the argument of $E$ denotes the fact that it is a function of the full vector $\mathbf{Q} \in \mathbb{R}^{3 N}$. The Fokker-Planck equation for (3.1) is

$$
\partial_{t} \rho=-\nabla_{\mathbf{Q}} \cdot(\mathbf{V} \rho)+M^{-1} \nabla_{\mathbf{V}} \cdot(\nabla E(\mathbf{Q}) \rho)+M^{-1} \nabla_{\mathbf{V}} \cdot[\gamma(\mathbf{V}-A \mathbf{Q}) \rho]+\frac{1}{2} M^{-2} \sigma^{2} \Delta_{\mathbf{V}} \rho .
$$

We do not have an analytic expression for solutions of the time-dependent equation (3.2) or any steady-state solutions. Thus, it is useful to carry out numerical experiments on the response of the multi-particle system to the background forcing and explore the resulting constitutive relation.

\subsection{Simple Lennard-Jones fluid}

We now run numerical tests on a 3D flow of particles with Lennard-Jones interactions and background shear flow

$$
A=\left[\begin{array}{lll}
0 & s & 0 \\
0 & 0 & 0 \\
0 & 0 & 0
\end{array}\right] .
$$

A closely related dynamics, differing in the choice of boundary conditions (we detail our choice below) and how the external forcing is handled, is considered in [12] where the authors perform rigorous asymptotic analysis on the invariant measure as well as numerical viscosity experiments.

We let $\Omega=[-L / 2, L / 2]^{3}$ be the computational domain, and we initially arrange $N$ particles on a uniform cubic lattice with spacing $a$, where $L=a N^{1 / 3}$. The initial velocities are random, drawn from the measure 
$Z^{-1} \exp \left(-\frac{\beta}{2}|\mathbf{V}-A \mathbf{Q}|^{2}\right)$. We note that over the long times that we simulate, the solution is insensitive to the choice of initial conditions (we have also tested with zero initial velocities). The potential $E$ denotes the Lennard-Jones potential energy with cutoff

$$
E(\mathbf{Q})=\sum_{i=1}^{N} \sum_{j=i+1}^{N} \phi\left(r_{i j}\right),
$$

where

$$
\phi(r)= \begin{cases}4 \varepsilon\left(\frac{1}{r^{12}}-\frac{1}{r^{6}}\right)+c_{1} r+c_{2} & \text { if } r<r_{\text {cut }}, \\ 0 & \text { if } r \geq r_{\text {cut }} .\end{cases}
$$

The constants $c_{1}$ and $c_{2}$ are chosen so that $\phi(r)$ and $\phi^{\prime}(r)$ are continuous at $r_{\text {cut }}$. The distance $r_{i j}=\left|\mathbf{Q}_{i}-\mathbf{Q}_{j}\right|$ is computed taking into account the boundary conditions which we now make precise.

We apply the Lees-Edwards boundary conditions $[1,9]$ to the system, which generalize standard periodic boundary conditions to be consistent with shear flow. At time $t$, the particle $(\mathbf{Q}(t), \mathbf{V}(t))$ has periodic replicas

$$
\begin{aligned}
& \left(Q_{1}(t), Q_{2}(t), Q_{3}(t), V_{1}(t), V_{2}(t), V_{3}(t)\right) \\
& \quad=\left(Q_{1}(t)+m L+t n s L, Q_{2}(t)+n L, Q_{3}(t)+k L, V_{1}(t)+n s L, V_{2}(t), V_{3}(t)\right)
\end{aligned}
$$

for all $m, n, k \in \mathbb{Z}$. This ensures that the periodic replicas of the system move consistently with the shear.

We first perform a single run with the parameters $\varepsilon=1, N=1000, \beta=1.0, s=0.05, M=1, a=$ $0.7^{-1 / 3}, r_{\text {cut }}=2.6, \gamma=1, \sigma=\sqrt{2 \gamma \beta^{-1}}$. For this choice of parameters, the Lennard-Jones particles are in a fluid regime (see e.g. [22]). We run the simulation up to time $T=500$, with a stepsize of $\Delta t=0.005$ using the following splitting algorithm. We let $\left(\mathbf{Q}^{n}, \mathbf{V}^{n}\right)$ denote the approximate particle position and velocity at time $t=n \Delta t$. We let $\alpha=\mathrm{e}^{-\frac{\gamma}{M} \Delta t}$, and for $i=1, \ldots, N$ we define $\left(\mathbf{Q}_{i}^{n+1}, \mathbf{V}_{i}^{n+1}\right)$ by

$$
\begin{aligned}
\mathbf{V}_{i}^{n+1 / 2} & =\mathbf{V}_{i}^{n}-\frac{\Delta t}{2} M^{-1} \nabla_{\mathbf{Q}_{i}} E\left(\mathbf{Q}^{n}\right) \\
\mathbf{Q}_{i}^{n+1} & =\mathbf{Q}_{i}^{n}+\Delta t \mathbf{V}_{i}^{n+1 / 2} \\
\mathbf{V}_{i}^{*} & =\mathbf{V}_{i}^{n+1 / 2}-\frac{\Delta t}{2} M^{-1} \nabla_{\mathbf{Q}_{i}} E\left(\mathbf{Q}^{n+1}\right) \\
\mathbf{V}_{i}^{n+1} & =\alpha \mathbf{V}_{i}^{*}+(1-\alpha) A \mathbf{Q}_{i}^{n+1}+\left(\frac{1-\alpha^{2}}{\beta M}\right)^{1 / 2} \mathbf{G}_{i}^{n}
\end{aligned}
$$

where $\mathbf{G}_{i}^{n} \sim \mathcal{N}(0, I)$ where $I$ denotes the $d \times d$ identity matrix. This is a composition of a Verlet step applied to the Hamiltonian portion

$$
\begin{aligned}
\mathrm{d} \mathbf{Q}_{i} & =\mathbf{V}_{i} \mathrm{~d} t, \\
M \mathrm{~d} \mathbf{V}_{i} & =-\nabla_{\mathbf{Q}_{i}} E(\mathbf{Q}) \mathrm{d} t
\end{aligned}
$$

with an exact integration of the remaining terms which represent the effect of the heat bath on the large particles,

$$
\begin{aligned}
\mathrm{d} \mathbf{Q}_{i} & =0, \\
M \mathrm{~d} \mathbf{V}_{i} & =-\gamma\left(\mathbf{V}_{i}-A \mathbf{Q}_{i}\right) \mathrm{d} t+\sigma \mathrm{d} \mathbf{W}_{i} .
\end{aligned}
$$

\subsection{Mean flow and stress}

In Figure 5 we display the results of our numerical simulation of shear flow. We partition the domain into $K=100$ slices,

$$
R_{k}=[-L / 2, L / 2] \times\left[-L / 2+\frac{k-1}{K} L,-L / 2+\frac{k}{K} L\right] \times[-L / 2, L / 2]
$$


(a)

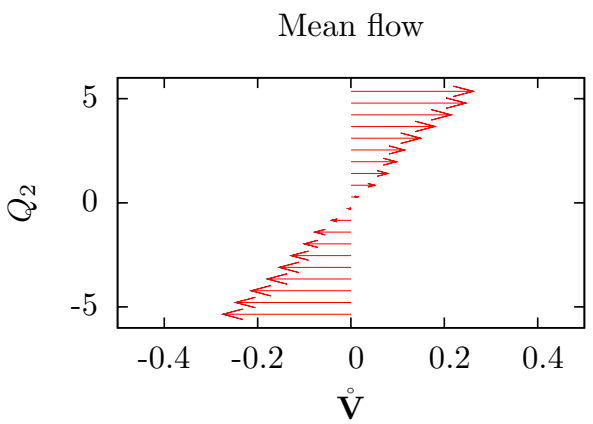

(c)

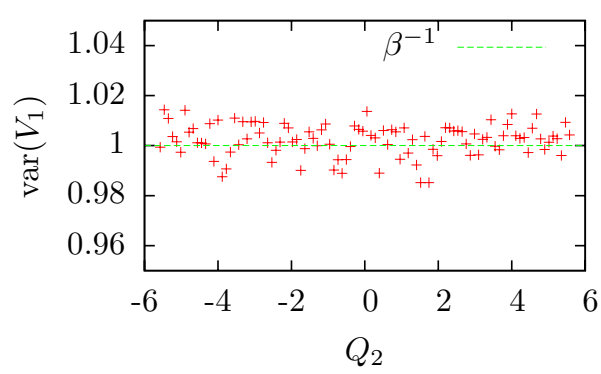

(e)

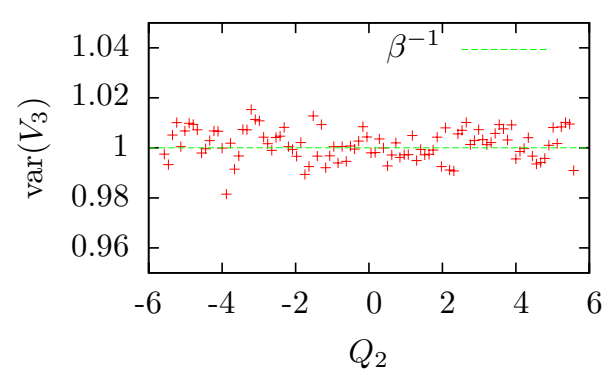

(b)

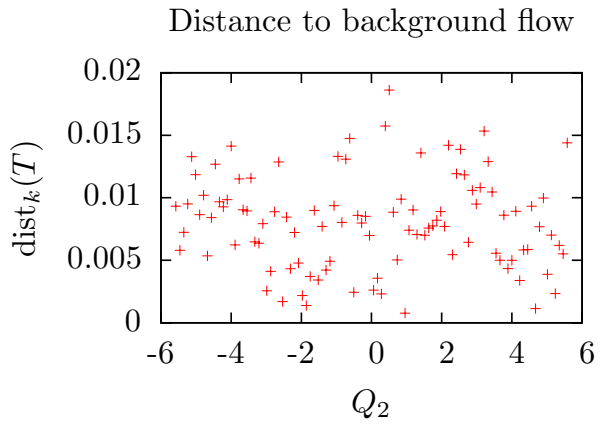

(d)

Variance in $V_{2}$

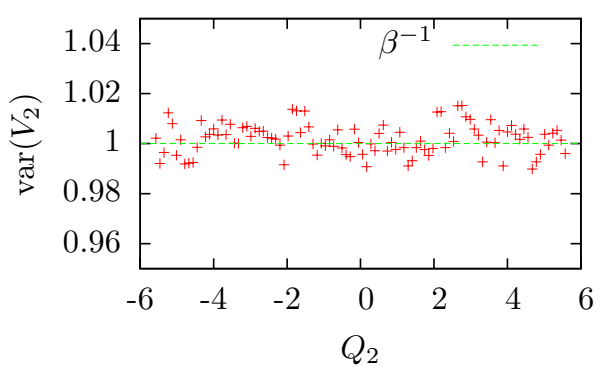

(f)

Convergence of mean flow to background

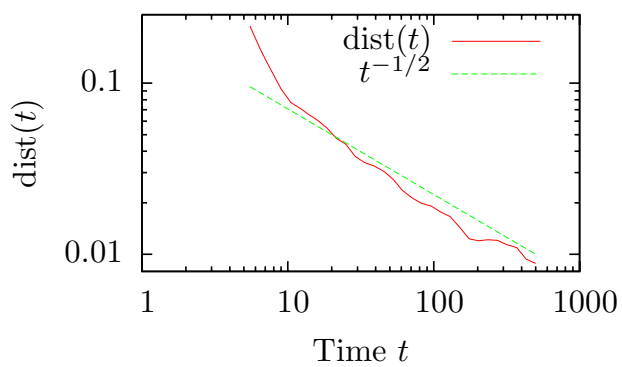

Figure 5. (a) The mean velocity (3.4) of the particles within horizontal slices (3.3) for a shear flow with strain rate $s=0.05$, averaged in $t$. We plot 20 of the 100 bins used in creating the statistics. (b) The distance (3.5) between the computed mean velocity and the background flow is plotted versus $Q_{2}$ at the final time $t_{\ell}=T=500$. (c), (d), and (e) The variance of particle velocity around the mean is plotted versus $Q_{2}$, where we again average in $Q_{1}, Q_{3}$, and $t$. This is well centered around $\beta^{-1}$. (f) The distance (3.6) of the computed mean velocity to the background flow is plotted versus time in a log-log axis. We observe the expected $\mathrm{O}\left(t^{-1 / 2}\right)$ decay of the distance.

for $k=1, \ldots, K$. At any time $t_{\ell}=\ell \Delta t$, we define a time average of the mean velocity in each slice $k$ by

$$
\stackrel{\circ}{\mathbf{V}}\left(t_{\ell}, k\right)=\frac{\sum_{n=0}^{\ell} \sum_{i=1}^{N} \mathbf{V}_{i}^{n} \mathbb{1}_{R_{k}}\left(\mathbf{Q}_{i}^{n}\right)}{\sum_{n=0}^{\ell} \sum_{i=1}^{N} \mathbb{1}_{R_{k}}\left(\mathbf{Q}_{i}^{n}\right)}
$$

where $\mathbf{V}_{i}^{n}$ denotes the velocity of particle $i$ at time $n \Delta t$ and where $\mathbb{1}_{R_{k}}$ denotes the indicator function of the slice $R_{k}$. Since the flow is uniform in all but the $\mathbf{e}_{2}$-direction, averaging on slices helps improve the convergence 


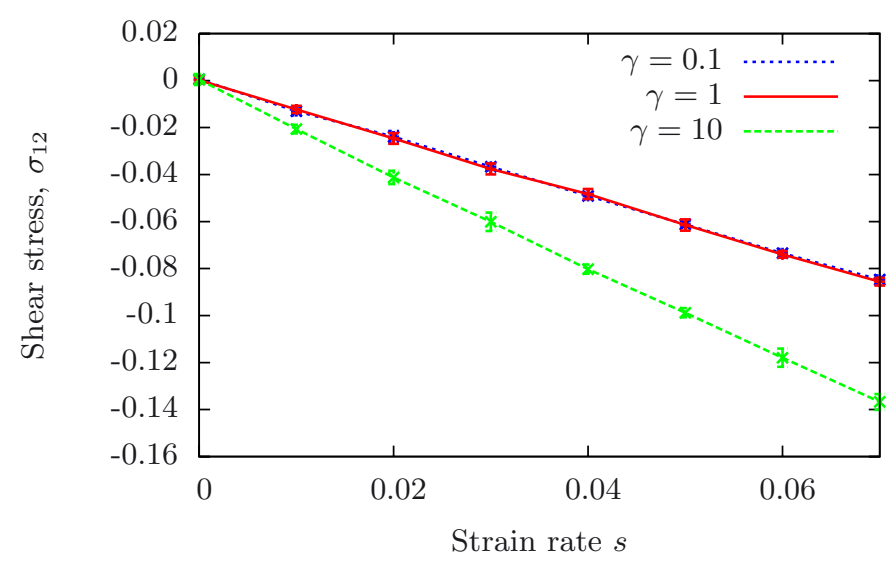

FIGURE 6 . Shear stress $\sigma_{12}$ vs velocity gradient. We observe that the viscosity increases with the heat bath parameter $\gamma$, though for the two smaller values of $\gamma$, the viscosity is extremely close. For the values $\gamma=0.1, \gamma=1.0$, and $\gamma=10$, we find viscosities $\eta=1.2175, \eta=1.2254$, and $\eta=1.9518$, respectively.

of the flow statistics. The time average over the full simulation of the mean velocity is plotted in Figure 5a, and we observe a linear profile equal to the applied background flow.

We define the distance of the mean flow in each slice to the background flow,

$$
\operatorname{dist}\left(t_{\ell}, k\right)=\left(\left(\stackrel{\circ}{V}_{1}\left(t_{\ell}, k\right)-s Q_{2}(k)\right)^{2}+\stackrel{\circ}{V}_{2}^{2}\left(t_{\ell}, k\right)+\stackrel{\circ}{V}_{3}^{2}\left(t_{\ell}, k\right)\right)^{1 / 2},
$$

where $Q_{2}(k)=-L / 2+\frac{k-1 / 2}{K} L$ is the $y$-coordinate of the center of rectangle $R_{k}$. We plot the distance as a function of $Q_{2}$ in Figure $5 \mathrm{~b}$ and we observe that it is uniform throughout the domain. The variance of the velocity is computed for each slice and is displayed in Figure $5 \mathrm{c}$, d, and e. The variance closely matches the expected variance due to the background temperature, $\frac{1}{k_{B} \beta}=1$. In particular, it is statistically the same in each direction so that we observe a scalar temperature in contrast to the laminar flow case of (2.13). In Figure $5 \mathrm{f}$, we plot the distance of the mean flow $\dot{\mathbf{V}}\left(t_{\ell}, k\right)$ to the background flow versus time,

$$
\operatorname{dist}\left(t_{\ell}\right)=\left(\frac{1}{K} \sum_{k=1}^{K}\left(\stackrel{\circ}{V}_{k, 1}\left(t_{\ell}\right)-s Q_{k, 2}\right)^{2}+\stackrel{\circ}{V}_{k, 2}^{2}+\stackrel{\circ}{V}_{k, 3}^{2}\right)^{1 / 2} .
$$

We observe an $\mathrm{O}\left(t^{-1 / 2}\right)$ decay in the distance, which is the convergence rate to the mean expected for Monte Carlo empirical averages.

In Figure 6, we display the $\sigma_{12}$ term of the shear stress, computed using the Irving-Kirkwood formula [11] which we average over all time steps

$$
\boldsymbol{\sigma}=\frac{1}{|\Omega|} \sum_{i=1}^{N}\left(M\left(\mathbf{V}_{i}^{n}-A \mathbf{Q}_{i}^{n}\right) \otimes\left(\mathbf{V}_{i}^{n}-A \mathbf{Q}_{i}^{n}\right)+\frac{1}{2} \sum_{\substack{j=1 \\ j \neq i}}^{N}\left(\mathbf{Q}_{i}-\mathbf{Q}_{j}\right) \otimes f^{(i j)}\right)
$$

where

$$
f^{(i j)}=-\frac{\phi^{\prime}\left(\left|\mathbf{Q}_{i}-\mathbf{Q}_{j}\right|\right)\left(\mathbf{Q}_{i}-\mathbf{Q}_{j}\right)}{\left|\mathbf{Q}_{i}-\mathbf{Q}_{j}\right|}
$$


denotes the force on atom $i$ due to atom $j$ and $|\Omega|=L^{3}$ is the volume of $\Omega$. We note that it is standard to subtract the local average velocity from the pressure term [11]: hence we have subtracted the background flow at each step. We run ten independent simulations for each choice of the strain rate $s=0,0.01,0.02, \ldots, 0.07$ and the parameter $\gamma=0.1,1.0,10$. The simulations are all run to time $T=500$, with $N, \beta, M, a, r_{\text {cut }}$, and $\sigma$ chosen as above. We plot the stress along with error bars equal to 3 times the standard deviation. Time averages of the shear stress $\sigma_{12}$ are plotted against the strain rate $s$. We notice a linear relation, and define the shear viscosity $\eta=-\frac{\sigma_{12}}{s}$. Fitting the data, we find viscosity (with standard deviation) $\eta=1.2175 \pm 0.0085$ for $\gamma=0.1$ whose confidence interval overlaps with the value $\eta=1.142 \pm 0.087$ reported in [22], Table IV. The algorithm successfully simulates a system out of equilibrium, and the computed viscosity is consistent with previous computations for Lennard--Jones fluids.

\section{Appendix A. Convergence in the small mass limit}

In this section, we outline the proof of the convergence of the mechanical process $\left(\mathbf{Q}_{m}, \mathbf{V}_{m}\right)$ to the solution of the nonequilibrium Langevin dynamics (2.21), as stated in Theorem 2.4. We construct a Markov approximation $\left(\widetilde{\mathbf{Q}}_{m}, \widetilde{\mathbf{V}}_{m}\right)$ that only counts the "fast collisions" of the mechanical process (which we define below). The Markov approximation acts as an intermediate process between the mechanical process and the nonequilibrium Langevin dynamics. We prove in Appendix B that the Markov approximation $\left(\widetilde{\mathbf{Q}}_{m}, \widetilde{\mathbf{V}}_{m}\right)$ converges to the solution of the nonequilibrium Langevin dynamics (2.21) in the sense that

$$
\left(\widetilde{\mathbf{Q}}_{m}, \widetilde{\mathbf{V}}_{m}\right)_{\{0 \leq t \leq T\}} \stackrel{\mathcal{L}}{\longrightarrow}(\mathbf{Q}, \mathbf{V})_{\{0 \leq t \leq T\}} \text { as } m \rightarrow 0 .
$$

In Appendix D we show that the mechanical process and Markov approximation are close to each other, in the sense that

$$
\left(\widetilde{\mathbf{Q}}_{m}, \widetilde{\mathbf{V}}_{m}\right)_{\{0 \leq t \leq T\}}-\left(\mathbf{Q}_{m}, \mathbf{V}_{m}\right)_{\{0 \leq t \leq T\}} \stackrel{p}{\longrightarrow} 0 \text { as } m \rightarrow 0 .
$$

This is shown by coupling the Markov process to the mechanical process, that is for each realization of the mechanical process we associate a (random) set of realizations of the Markov process that are (with high probability) close to the realization of the mechanical process. By a standard result in probability theory (see Thm. 3.1 of [2]), properties (A.1) and (A.2) allow us to conclude Theorem 2.4, that is, that

$$
\left(\mathbf{Q}_{m}, \mathbf{V}_{m}\right)_{\{0 \leq t \leq T\}} \stackrel{\mathcal{L}}{\longrightarrow}(\mathbf{Q}, \mathbf{V})_{\{0 \leq t \leq T\}} \text { as } m \rightarrow 0 .
$$

The proof here is structured as in [7], with changes made to handle the fact that the distribution of the initial bath atom velocities depends on space. We work with the pairing $\left(\mathbf{Q}_{m}, \mathbf{V}_{m}\right)$, since in our case, we cannot create a Markov approximation for the velocity alone. We give a self-contained proof, while also pointing out where it differs from the original argument in [7].

\section{A.1. Rate of fast collisions}

As noted in Remark 2.1, the mechanical process is not a Markov process itself since a bath atom may collide more than once with the large particle or certain collisions may be impossible due to past motion of the particle. However, the expected value of the relative speed of a bath atom is $O\left(m^{-1 / 2}\right)$, which is large since $m$ is assumed small, whereas the expected value of the relative speed of the large particle is much smaller, $O(1)$. Most collisions happen between a fast-moving bath atom and a slower-moving large particle, and we show below that for such collisions there is no chance of recollision between the large particle and the bath atom involved. This motivates the following definition of "fast collisions" and the introduction below of a stopping time on the trajectory of the mechanical process that controls the large particle's velocity and position. 
Definition A.1. We call a collision a fast collision if the normal component of the relative velocity (2.19) of the bath atom before the collision occurs satisfies

$$
\bar{v}_{\mathrm{n}}>c_{m}:=m^{-1 / 5}
$$

where $\bar{v}_{\mathrm{n}}=\overline{\mathbf{v}} \cdot \mathbf{e}_{\mathrm{n}}$. Every other collision is called a slow collision.

The particular scaling of $c_{m}$ is chosen so that $c_{m} \rightarrow \infty$ and $c_{m}^{2} m^{1 / 2} \rightarrow 0$ as $m \rightarrow 0$. We use the second limit in Appendix D.4 to bound the total effect of slow collisions.

Definition A.2. Let $T>0$ be given. For a given realization of the mechanical process $\left(\mathbf{Q}_{m}, \mathbf{V}_{m}\right)$, we define the stopping time

$$
\tau_{m}=\min \left(\inf _{t \geq 0}\left\{t:\|A\|\left|\mathbf{Q}_{m}(t)\right|+\left|\mathbf{V}_{m}(t)\right| \geq c_{m} / 8\right\}, T\right) .
$$

In the following, we assume that $m$ is sufficiently small so that the initial condition satisfies $\|A\||\mathbf{Q}(0)|+|\mathbf{V}(0)|<$ $c_{m} / 8$, where we recall that the initial condition is independent of $m$.

In Appendix E we show that almost surely $\tau_{m}>0$ and that the mechanical process is well-posed up to $\tau_{m}$. In particular we show that almost surely, before time $\tau_{m}$ there are only finitely many collisions and none of these collisions involve more than one bath atom at the same time. Intuitively, this means that the fast collisions have no memory of the large particle's trajectory, and furthermore it means that the rate of fast collisions is governed only by the initial bath configuration (2.16). In Appendix C, we show that if a bath atom experiences a fast collision with the mechanical process's large particle sometime in the interval $\left[0, \tau_{m}\right)$, then this is the only collision that the bath atom undergoes in $\left[0, \tau_{m}\right)$. From the coupling that we construct in Appendix D between the mechanical process and the Markov approximation and a consideration of the expected size of the Markov approximation ((D.9) and (D.10)), we can show that $\lim _{m \rightarrow 0} \mathbb{P}\left(\tau_{m}=T\right)=1$, so that for the sake of our convergence proof, on our time interval of interest, $[0, T]$, fast collisions do not introduce memory terms. We thus define below a rate of collisions that the large particle experiences whenever the bath has configuration distributed according to (2.16).

Lemma A.3. Suppose that a large particle is at all times surrounded by a bath of atoms distributed according to (2.16). Then the instantaneous probability of a collision between the particle at position $\mathbf{Q}$ and velocity $\mathbf{V}$ and a bath atom with velocity in the ball $B(\mathbf{v} ; \mathrm{d} \mathbf{v})$ on the surface element $R^{d-1} \mathrm{~d} \Omega$ centered around $\mathbf{q}=\mathbf{Q}-R \mathbf{e}_{\mathrm{n}}$ in the time interval $[t, t+\mathrm{d} t]$ is given by

$$
r_{m}\left(\mathbf{v}, \mathbf{e}_{\mathrm{n}}, \mathbf{Q}, \mathbf{V}\right) \mathrm{d} \mathbf{v} \mathrm{d} \Omega \mathrm{d} t=\lambda_{m} R^{d-1} \max \left(v_{\mathrm{n}}-V_{\mathrm{n}}, 0\right) f_{m}\left(\mathbf{Q}-R \mathbf{e}_{\mathrm{n}}, \mathbf{v}\right) \mathrm{d} \mathbf{v} \mathrm{d} \Omega \mathrm{d} t
$$

where $R$ is the radius of the large particle, $\lambda_{m}=m^{-1 / 2} \lambda$ is the expected number of bath atoms per unit volume, $v_{\mathrm{n}}=\mathbf{v} \cdot \mathbf{e}_{\mathrm{n}}$ is the normal velocity of the incoming bath atom, and $V_{\mathrm{n}}=\mathbf{V} \cdot \mathbf{e}_{\mathrm{n}}$ is the normal velocity of the large particle.

Remark A.4. The assumption that the large particle always sees the same environment is too strong, and indeed does not hold for the large particle in the mechanical process. However, as we show in Appendix C, for bath atoms that undergo fast collisions with the large particle, their pre-collision distribution does satisfy (2.16). Thus, the rate (A.4) is correct for fast collisions of the mechanical process.

Proof. In Figure 7 we sketch the differential volume element of size $R^{d-1} \mathrm{~d} \Omega\left(v_{\mathrm{n}}-V_{\mathrm{n}}\right) \mathrm{d} t$ with base on the surface of the particle and height determined by the velocity of the incoming bath atom. The velocity of the bath atom and the large particle are constant on the time interval $\mathrm{d} t$, so this surface gives the infinitesimal rate $r_{m}$ of fast collisions. 


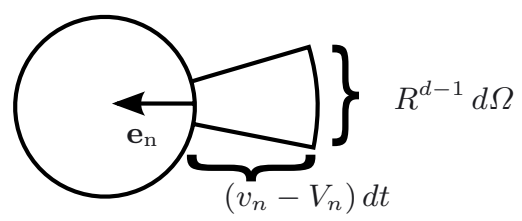

FiguRE 7. The differential area carved out by fast atoms that collide with the large particle in the time interval $[t, t+\mathrm{d} t]$, see (A.4).

\section{A.2. Rate of jumps and the Markov approximation}

We now use the rate (A.4) to define a rate for a Markov approximation to the mechanical process, where we only count the effect of fast collisions. We change variables to be in terms of jumps in velocity for the large particle rather than velocities of bath atoms. We first integrate out tangential directions $\mathbf{v}_{t}$ from $r_{m}$, and define the marginal

$$
f^{1}(x)=\int_{\mathbf{v}_{t}} f\left(x \mathbf{e}_{\mathrm{n}}+\mathbf{v}_{t}\right) \mathrm{d} \mathbf{v}_{t},
$$

where we recall that $f$ was assumed to be rotationally invariant, so that the left hand side does not depend on $\mathbf{e}_{\mathrm{n}}$. We define also the scaled marginal,

$$
f_{m}^{1}(x)=m^{1 / 2} f^{1}\left(m^{1 / 2} x\right) .
$$

Rewriting the collision rule (2.7), we have that the change in normal velocity of the large particle in the $\mathbf{e}_{\mathrm{n}^{-}}$ direction, $\widehat{V}=V_{\mathrm{n}}^{\prime}-V_{\mathrm{n}}$, due to a bath atom with normal velocity $\mathbf{v}_{\mathrm{n}}$ satisfies

$$
\widehat{V}=\frac{2 m}{M+m}\left(v_{\mathrm{n}}-V_{\mathrm{n}}\right) .
$$

We integrate out the tangential direction $\mathbf{v}_{t}$ from the rate $r_{m}$, make the change of variables from $v_{\mathrm{n}}$ to $\widehat{V}$ in (A.6), and introduce a Heaviside function to restrict to jumps caused by fast collisions. The rate on jumps is

$$
\begin{aligned}
\hat{r}_{m}\left(\widehat{V}, \mathbf{e}_{\mathrm{n}}, \mathbf{Q}, \mathbf{V}\right)= & \lambda_{m} R^{d-1}\left(\frac{M+m}{2 m}\right)^{2} H\left(\frac{M+m}{2 m} \widehat{V}+V_{\mathrm{n}}-(A \mathbf{q})_{\mathrm{n}}-c_{m}\right) \\
& \times \max (\widehat{V}, 0) f_{m}^{1}\left(\frac{M+m}{2 m} \widehat{V}+V_{\mathrm{n}}-(A \mathbf{q})_{\mathrm{n}}\right),
\end{aligned}
$$

where

$$
\mathbf{q}=\mathbf{Q}-R \mathbf{e}_{\mathrm{n}}
$$

represents the position on the large particle surface where the bath atom collides. Due to the Heaviside function, the minimum jump size $\widehat{V}$ with nonzero probability is

$$
\widehat{V}_{\text {min }}=\frac{2 m}{M+m} \max \left(\left(c_{m}-V_{\mathrm{n}}+(A \mathbf{q})_{\mathrm{n}}\right), 0\right),
$$

where we have kept implicit the dependence of $\widehat{V}_{\text {min }}$ on $\mathbf{Q}$ and $\mathbf{V}$. We let

$$
\Lambda_{m}(\mathbf{Q}, \mathbf{V})=\int_{\mathbb{S}^{d-1}} \int_{\widehat{V}_{\min }}^{\infty} \hat{r}_{m}\left(\widehat{V}, \mathbf{e}_{\mathrm{n}}, \mathbf{Q}, \mathbf{V}\right) \mathrm{d} \widehat{V} \mathrm{~d} \Omega
$$


denote the jump intensity, and a short calculation shows that $\Lambda_{m}(\mathbf{Q}, \mathbf{V})$ is independent of $(\mathbf{Q}, \mathbf{V})$ whenever

$$
|| A|||\mathbf{Q}|+|\mathbf{V}| \leq c_{m} / 8
$$

holds, due to the Heaviside function in $\hat{r}_{m}$.

Definition A.5. We define the Markov process $\left(\widetilde{\mathbf{Q}}_{m}, \widetilde{\mathbf{V}}_{m}\right)$ starting from initial condition $\left(\widetilde{\mathbf{Q}}_{m}(0), \widetilde{\mathbf{V}}_{m}(0)\right)=$ $\left(\mathbf{Q}_{m}(0), \mathbf{V}_{m}(0)\right)$ to be a Poisson jump process where the velocity experiences jumps $\widehat{V} \mathbf{e}_{\mathrm{n}}$ with intensity $\Lambda_{m}(0,0)$ and with probability density function

$$
\Lambda_{m}(0,0)^{-1} \hat{r}_{m}\left(\widehat{V}, \mathbf{e}_{\mathrm{n}}, \widetilde{\mathbf{Q}}_{m}, \widetilde{\mathbf{V}}_{m}\right),
$$

and between jumps, $(\widetilde{\mathbf{Q}}, \widetilde{\mathbf{V}})$ evolve according to $(2.6)$.

\section{Appendix B. Convergence of the Markov approximation to the SDE}

To show Theorem 2.4, we first show that the Markov approximation $\left(\widetilde{\mathbf{Q}}_{m}, \widetilde{\mathbf{V}}_{m}\right)$, with rate given by (A.7), converges in law to (2.21). We write the generator for the Markov approximation and show that it converges as $m \rightarrow 0$ to the generator for (2.21). These calculations are carried out explicitly below, allowing us to get the coefficients for the SDE in terms of the parameters of the heat bath and the large particle. The setup and calculations follow as in [7]. Because of the $\mathbf{Q}$-dependence of the process, we work with test functions $\psi(\mathbf{Q}, \mathbf{V})$ that are functions of the position and velocity. The inhomogeneity of the velocity field does not change the coefficients $\gamma$ and $\sigma$ compared to the zero background flow case (2.9).

Lemma B.1. Let $T>0$ be given. The Markov process $\left(\widetilde{\mathbf{Q}}_{m}, \widetilde{\mathbf{V}}_{m}\right)$ satisfies

$$
\left(\widetilde{\mathbf{Q}}_{m}, \widetilde{\mathbf{V}}_{m}\right)_{\{0 \leq t \leq T\}} \stackrel{\mathcal{L}}{\longrightarrow}(\mathbf{Q}, \mathbf{V})_{\{0 \leq t \leq T\}} \quad \text { as } m \rightarrow 0
$$

on $[0, T]$, where $(\mathbf{Q}, \mathbf{V})$ satisfies the nonequilibrium Langevin dynamics (2.21).

For a stochastic process, we let $\mathrm{U}_{t}: C_{0}\left(\mathbb{R}^{2 d} ; \mathbb{R}\right) \rightarrow C_{0}\left(\mathbb{R}^{2 d} ; \mathbb{R}\right)$ denote the evolution semigroup, where $C_{0}\left(\mathbb{R}^{2 d} ; \mathbb{R}\right)$ denotes the set of continuous functions with zero limit at infinity. We define the infinitesimal generator $L$ by

$$
L \psi(\mathbf{Q}, \mathbf{V})=\lim _{t \rightarrow 0} \frac{\mathrm{U}_{t} \psi(\mathbf{Q}, \mathbf{V})-\psi(\mathbf{Q}, \mathbf{V})}{t}
$$

where the domain of $L$ is the set of $C_{0}$ functions such that the above limit exists in $C_{0}$. In the following, we use $L_{m}$ to denote the infinitesimal generator for the Markov approximation $\left(\widetilde{\mathbf{Q}}_{m}, \widetilde{\mathbf{V}}_{m}\right)$ and $L$ for the nonequilibrium Langevin dynamics (2.21). We prove Lemma B.1 with the use of Lemma B.2 below, which is a general lemma relating convergence of generators to convergence in law of the process. We recall that $\mathcal{D}([0, T])$ represents the space of càdlàg functions on the interval $[0, T]$. A linear subspace $K$ of the domain of $L$ is called a core for $L$ if $L$ is the closure of $\left.L\right|_{K}$ [14]. One can show that $C_{c}^{\infty}$, the set of compactly supported $C^{\infty}$ functions, is a core for the nonequilibrium Langevin dynamics (2.21) using the ideas of [14].

The process $(\mathbf{Q}, \mathbf{V})$ is a Markov- $C_{0}$-process, which means its evolution semigroup satisfies $\mathrm{U}_{t} C_{0} \subset C_{0}$ and $\lim _{t \rightarrow 0}\left\|\mathrm{U}_{t} \psi-\psi\right\|=0$ for all $\psi \in C_{0}$.

Lemma B.2. Consider a family of Markov processes $Z_{m}$ on $\mathcal{D}([0, T])$ with generators $L_{m}$. Suppose that the Markov- $C_{0}$-process $Z$ has generator $L$. Let $K$ be a core for $L$ such that $\psi \in K \Longrightarrow \psi \in \mathcal{D}\left(L_{m}\right)$ for all sufficiently small $m$. Suppose that the initial distribution of $Z_{m}$ converges weakly to that of $Z$ and that

$$
\forall \psi \in K, \quad \lim _{m \rightarrow 0} \sup _{z \in \mathbb{R}^{2 d}}\left|L_{m} \psi(z)-L \psi(z)\right|=0 .
$$

Then

$$
Z_{m} \stackrel{\mathcal{L}}{\longrightarrow} Z \quad \text { as } \quad m \rightarrow 0 .
$$

The above lemma is Lemma 4.1 of [7], which can be found in similar form in [14] or [23]. 


\section{B.1. Generator for the Markov process}

We apply the generator $L_{m}$ for the Markov process to a $C_{c}^{\infty}\left(\mathbb{R}^{2 d}\right)$ test function, expand in powers of $m$, and show that we have, to leading order, a drift and diffusion term.

Applying (B.1) to the Markov process, we have for any $\psi(\mathbf{Q}, \mathbf{V}) \in C_{c}^{\infty}\left(\mathbb{R}^{2 d}\right)$ that the generator satisfies

$$
L_{m} \psi(\mathbf{Q}, \mathbf{V})=\mathbf{V} \cdot \nabla_{\mathbf{Q}} \psi(\mathbf{Q}, \mathbf{V})+\int_{\mathbb{S}^{d-1}} \int_{\widehat{V}_{\min }}^{\infty}\left[\psi\left(\mathbf{Q}, \mathbf{V}+\widehat{V} \mathbf{e}_{\mathrm{n}}\right)-\psi(\mathbf{Q}, \mathbf{V})\right] \hat{r}_{m}\left(\widehat{V}, \mathbf{e}_{\mathrm{n}}, \mathbf{Q}, \mathbf{V}\right) \mathrm{d} \widehat{V} \mathrm{~d} \Omega
$$

where $\hat{r}_{m}$ is defined in (A.7) and $\widehat{V}_{\text {min }}$, which we note depends on $\mathbf{e}_{\mathrm{n}}$, is defined in (A.8). We recall, from the scaling of bath atom velocities $(2.17)$, that $\mathbb{E}_{m}(|\mathbf{v}|)$ is $O\left(m^{-1 / 2}\right)$. From (A.6) this scaling implies that $\mathbb{E}_{m}(\widehat{V})$ is $O\left(m^{1 / 2}\right)$. To find the limiting generator in the limit $m \rightarrow 0$, we expand in powers of $\widehat{V}$ and write

$$
\begin{aligned}
\psi\left(\mathbf{Q}, \mathbf{V}+\widehat{V} \mathbf{e}_{\mathrm{n}}\right)-\psi(\mathbf{Q}, \mathbf{V})= & \widehat{V} \mathbf{e}_{\mathrm{n}} \cdot \nabla_{\mathbf{V}} \psi(\mathbf{Q}, \mathbf{V})+\frac{1}{2} \widehat{V}^{2}\left(\mathbf{e}_{\mathrm{n}} \otimes \mathbf{e}_{\mathrm{n}}\right): \nabla_{\mathbf{V}}^{2} \psi(\mathbf{Q}, \mathbf{V}) \\
& +\frac{1}{6} \widehat{V}^{3}\left(\mathbf{e}_{\mathrm{n}} \otimes \mathbf{e}_{\mathrm{n}} \otimes \mathbf{e}_{\mathrm{n}}\right) \cdot 3 \cdot \nabla_{\mathbf{V}}^{3} \psi\left(\mathbf{Q}, \mathbf{V}+\widehat{V}^{*} \mathbf{e}_{\mathrm{n}}\right),
\end{aligned}
$$

for some $\widehat{V}^{*} \in(0, \widehat{V})$ and where $\cdot 3 \cdot$ denotes the third-order contraction product, which we apply to the 3 -tensors $\left(\mathbf{e}_{\mathrm{n}} \otimes \mathbf{e}_{\mathrm{n}} \otimes \mathbf{e}_{\mathrm{n}}\right)$ and $\nabla_{\mathbf{V}}^{3}$. We then write

$$
L_{m} \psi(\mathbf{Q}, \mathbf{V})=\mathbf{V} \cdot \nabla_{\mathbf{Q}} \psi(\mathbf{Q}, \mathbf{V})+C_{m}\left(I_{1} \cdot \nabla_{\mathbf{V}} \psi(\mathbf{Q}, \mathbf{V})+\frac{1}{2} I_{2}: \nabla_{\mathbf{V}}^{2} \psi(\mathbf{Q}, \mathbf{V})+\mathcal{R}_{m}\right)
$$

where, recalling that $\mathbf{q}=\mathbf{Q}-R \mathbf{e}_{\mathrm{n}}$, we have

$$
\begin{aligned}
I_{1} & =m^{-5 / 2} \int_{\mathbb{S}^{d-1}} \int_{\widehat{V}_{\min }}^{\infty} \widehat{V}^{2} \mathbf{e}_{\mathrm{n}} f_{m}^{1}\left(\frac{M+m}{2 m} \widehat{V}+(\mathbf{V}-A \mathbf{q})_{\mathrm{n}}\right) \mathrm{d} \widehat{V} \mathrm{~d} \Omega \\
I_{2} & =m^{-5 / 2} \int_{\mathbb{S}^{d-1}} \int_{\widehat{V}_{\min }}^{\infty} \widehat{V}^{3}\left(\mathbf{e}_{\mathrm{n}} \otimes \mathbf{e}_{\mathrm{n}}\right) f_{m}^{1}\left(\frac{M+m}{2 m} \widehat{V}+(\mathbf{V}-A \mathbf{q})_{\mathrm{n}}\right) \mathrm{d} \widehat{V} \mathrm{~d} \Omega \\
\mathcal{R}_{m} & =\frac{m^{-5 / 2}}{6} \int_{\mathbb{S}^{d-1}} \int_{\widehat{V}_{\text {min }}}^{\infty} \widehat{V}^{4}\left(\mathbf{e}_{\mathrm{n}} \otimes \mathbf{e}_{\mathrm{n}} \otimes \mathbf{e}_{\mathrm{n}}\right) \cdot 3 \cdot \nabla_{\mathbf{V}}^{3} \psi\left(\mathbf{Q}, \mathbf{V}+\widehat{V}^{*} \mathbf{e}_{\mathrm{n}}\right) f_{m}^{1}\left(\frac{M+m}{2 m} \widehat{V}+(\mathbf{V}-A \mathbf{q})_{\mathrm{n}}\right) \mathrm{d} \widehat{V} \mathrm{~d} \Omega,
\end{aligned}
$$

with coefficient

$$
C_{m}=\lambda R^{d-1}\left(\frac{M+m}{2}\right)^{2} .
$$

Note that in our notation, we have suppressed the dependence of $I_{1}, I_{2}$, and $\mathcal{R}_{m}$ on $(\mathbf{Q}, \mathbf{V})$.

\section{B.1.1. Remainder term}

We begin with the remainder term $\mathcal{R}_{m}$. Since the test function $\psi$ belongs to $C_{c}^{\infty}$, we can restrict to the case where $\mathbf{Q}$ and $\mathbf{V}$ are bounded, and we may assume $m$ is sufficiently small so that $|\mathbf{V}|+\|A\||\mathbf{Q}| \leq c_{m}$. This, along with the finiteness of the moments (2.3), allows us to estimate the order of $\mathcal{R}_{m}$. Letting $x=$ $\left(\frac{M+m}{2 m^{1 / 2}} \widehat{V}+m^{1 / 2}(\mathbf{V}-A \mathbf{q})_{\mathrm{n}}\right)$, we compute

$$
\begin{aligned}
\left|\mathcal{R}_{m}\right| & \leq C m^{-2} \int_{\mathbb{S}^{d-1}} \int_{\widehat{V}_{\min }}^{\infty} \widehat{V}^{4}\left|\nabla_{\mathbf{V}}^{3} \psi\left(\mathbf{Q}, \mathbf{V}+\widehat{V}^{*} \mathbf{e}_{n}\right)\right| f^{1}\left(\frac{M+m}{2 m^{1 / 2}} \widehat{V}+m^{1 / 2}(\mathbf{V}-A \mathbf{q})_{\mathrm{n}}\right) \mathrm{d} \widehat{V} \mathrm{~d} \Omega \\
& \leq \frac{C m^{1 / 2}}{(M+m)^{5}} \int_{\mathbb{S}^{d-1}} \int_{m^{1 / 2} c_{m}}^{\infty}\left(x-m^{1 / 2}(\mathbf{V}-A \mathbf{q})_{\mathrm{n}}\right)^{4}\left|\nabla_{\mathbf{V}}^{3} \psi\left(\mathbf{Q}, \mathbf{V}+\widehat{V}^{*} \mathbf{e}_{n}\right)\right| f^{1}(x) \mathrm{d} x \mathrm{~d} \Omega
\end{aligned}
$$


We note that since we have assumed that $m$ is sufficiently small, the minimum value of $x$ is given by $x_{\min }=$ $m^{1 / 2} c_{m}$. Recalling that $\psi$ is compactly supported and that $\widehat{V}^{*} \in(0, \widehat{V})=\left(0,\left(\frac{2 m^{1 / 2}}{M+m}\right)\left(x-m^{1 / 2}(\mathbf{V}-A \mathbf{q})_{\mathrm{n}}\right)\right)$, we can bound $\mathbf{Q}, \mathbf{V}$ in the innermost integrand to find the estimate $\left(x-m^{1 / 2}(\mathbf{V}-A \mathbf{q})_{\mathrm{n}}\right)^{4}\left|\nabla_{\mathbf{V}}^{3} \psi\left(\mathbf{Q}, \mathbf{V}+\widehat{V}^{*} \mathbf{e}_{n}\right)\right| \leq$ $C(1+x)^{4}$ where $C$ may depend on $\psi$ but not on $m$. We find an upper bound on $\mathcal{R}_{m}$ by extending the interval of integration and using the boundedness of the marginals (2.3),

$$
\left|\mathcal{R}_{m}\right| \leq C \frac{m^{1 / 2}}{(M+m)^{5}} \int_{0}^{\infty}(1+x)^{4} f(x) \mathrm{d} x \leq C m^{1 / 2}
$$

\section{B.1.2. Diffusion coefficient}

We next turn to $I_{2}$, and compute

$$
\begin{aligned}
I_{2} & =m^{-5 / 2} \int_{\mathbb{S}^{d-1}} \int_{\widehat{V}_{\text {min }}}^{\infty}\left(\mathbf{e}_{\mathrm{n}} \otimes \mathbf{e}_{\mathrm{n}}\right) \widehat{V}^{3} f_{m}^{1}\left(\frac{M+m}{2 m} \widehat{V}+(\mathbf{V}-A \mathbf{q})_{\mathrm{n}}\right) \mathrm{d} \widehat{V} \mathrm{~d} \Omega \\
& =m^{-2} \int_{\mathbb{S}^{d-1}} \int_{\widehat{V}_{\text {min }}}^{\infty}\left(\mathbf{e}_{\mathrm{n}} \otimes \mathbf{e}_{\mathrm{n}}\right) \widehat{V}^{3} f^{1}\left(\frac{M+m}{2 m^{1 / 2}} \widehat{V}+m^{1 / 2}(\mathbf{V}-A \mathbf{q})_{\mathrm{n}}\right) \mathrm{d} \widehat{V} \mathrm{~d} \Omega .
\end{aligned}
$$

Let $x=\left(\frac{M+m}{2 m^{1 / 2}} \widehat{V}+m^{1 / 2}(\mathbf{V}-A \mathbf{q})_{\mathrm{n}}\right)$. We expand in powers of $m$, using the finiteness of the moments $(2.3)$ and the fact that $\psi$ is compactly supported, giving

$$
\begin{aligned}
I_{2} & =\frac{16}{(M+m)^{4}} \int_{\mathbb{S}^{d-1}} \int_{m^{1 / 2} c_{m}}^{\infty}\left(\mathbf{e}_{\mathrm{n}} \otimes \mathbf{e}_{\mathrm{n}}\right)\left(x-m^{1 / 2}(\mathbf{V}-A \mathbf{q})_{\mathrm{n}}\right)^{3} f^{1}(x) \mathrm{d} x \mathrm{~d} \Omega \\
& =\frac{16}{M^{4}} \int_{\mathbb{S}^{d-1}} \int_{0}^{\infty}\left(\mathbf{e}_{\mathrm{n}} \otimes \mathbf{e}_{\mathrm{n}}\right) x^{3} f^{1}(x) \mathrm{d} x \mathrm{~d} \Omega+O\left(m^{1 / 2}\right) \\
& =\frac{16}{M^{4}} \Phi_{3} \int_{\mathbb{S}^{d-1}}\left(\mathbf{e}_{\mathrm{n}} \otimes \mathbf{e}_{\mathrm{n}}\right) \mathrm{d} \Omega+O\left(m^{1 / 2}\right) \\
& =\frac{16 S_{d-1}}{M^{4} d} \Phi_{3} I+O\left(m^{1 / 2}\right)
\end{aligned}
$$

where $I$ denotes the $d \times d$ identity matrix and we recall that $S_{d-1}$ denotes the surface area of the $(d-1)$ sphere $\mathbb{S}^{d-1}$. The second line uses the estimate $\int_{0}^{m^{1 / 2} c_{m}} x^{3} f(x) \mathrm{d} x \leq m^{3 / 2} c_{m}^{3} \int_{\mathbb{R}} f(x) \mathrm{d} x=O\left(m^{1 / 2}\right)$ along with a gathering of higher order terms in $m$. The third line uses the definition (2.3) of $\Phi_{3}$, and the fourth line uses the following identity

$$
\int_{\mathbb{S}^{d-1}}\left(\mathbf{e}_{\mathrm{n}} \otimes \mathbf{e}_{\mathrm{n}}\right) \mathrm{d} \Omega=\frac{S_{d-1}}{d} I .
$$

Multiplying by $C_{m}$ of (B.4) and letting $m \rightarrow 0$ leads to the isotropic diffusion coefficient

$$
D I=\lim _{m \rightarrow 0} C_{m} I_{2}=\frac{4 \lambda R^{d-1} S_{d-1}}{M^{2} d} \Phi_{3} I .
$$

In the case of $f(v)=Z^{-1} \exp \left(-\frac{\beta v^{2}}{2}\right)$ with $Z=\frac{\sqrt{2 \pi}}{\sqrt{\beta}}$, we have $\Phi_{3}=\frac{\sqrt{2}}{\beta^{3 / 2} \sqrt{\pi}}$ and

$$
D=\frac{4 \sqrt{2} \lambda R^{d-1} S_{d-1}}{\beta^{3 / 2} \sqrt{\pi} M^{2} d} .
$$




\section{B.1.3. Drift coefficient}

We now similarly expand $I_{1}$ and find that the lowest order term, which is $O\left(m^{-1 / 2}\right)$, cancels out leaving an $O(1)$ drift term. Indeed, we have

$$
I_{1}=\int_{\mathbb{S}^{d-1}} \mathbf{e}_{\mathrm{n}} m^{-\frac{5}{2}} \int_{\widehat{V}_{\min }}^{\infty} \widehat{V}^{2} f_{m}^{1}\left(\frac{M+m}{2 m} \widehat{V}+V_{\mathrm{n}}-(A \mathbf{q})_{\mathrm{n}}\right) \mathrm{d} \widehat{V} \mathrm{~d} \Omega
$$

As before, we let $x=\frac{M+m}{2 m^{1 / 2}} \widehat{V}+m^{1 / 2}(\mathbf{V}-A \mathbf{q})_{\mathrm{n}}$. We obtain

$$
\begin{aligned}
I_{1} & =\frac{8 m^{-1 / 2}}{(M+m)^{3}} \int_{\mathbb{S}^{d-1}} \mathbf{e}_{\mathrm{n}} \int_{m^{1 / 2} c_{m}}^{\infty}\left(x-m^{1 / 2}(\mathbf{V}-A \mathbf{q})_{\mathrm{n}}\right)^{2} f^{1}(x) \mathrm{d} x \mathrm{~d} \Omega \\
& =\int_{\mathbb{S}^{d-1}} \mathbf{e}_{\mathrm{n}}\left[\frac{8 m^{-1 / 2}}{M^{3}} \int_{m^{1 / 2} c_{m}}^{\infty} x^{2} f^{1}(x) \mathrm{d} x+\frac{16}{M^{3}} \int_{0}^{\infty}\left(-x(\mathbf{V}-A \mathbf{q})_{\mathrm{n}}\right) f^{1}(x) \mathrm{d} x\right] \mathrm{d} \Omega+O\left(m^{3 / 10}\right),
\end{aligned}
$$

where the error terms are dominated by $\int_{0}^{m^{1 / 2} c_{m}} x f(x) \mathrm{d} x \leq m^{1 / 2} c_{m} \int_{\mathbb{R}} f(x) \mathrm{d} x=O\left(m^{3 / 10}\right)$. The first term vanishes,

$$
\int_{\mathbb{S}^{d-1}} \mathbf{e}_{\mathrm{n}} \int_{m^{1 / 2} c_{m}}^{\infty} x^{2} f^{1}(x) \mathrm{d} x \mathrm{~d} \Omega=0,
$$

since $\int_{\mathbb{S}^{d-1}} \mathbf{e}_{\mathrm{n}} \mathrm{d} \Omega=0$. We recall $\mathbf{q}=\mathbf{Q}-R \mathbf{e}_{\mathrm{n}}$, and let $\mathbf{W}=\mathbf{V}-A \mathbf{Q}$. We have the identities

$$
\begin{aligned}
& \int_{\mathbb{S}^{d-1}} \mathbf{e}_{\mathrm{n}} \cdot \mathbf{W} \mathbf{e}_{\mathrm{n}} \mathrm{d} \Omega=\int_{\mathbb{S}^{d-1}}\left(\mathbf{e}_{\mathrm{n}} \otimes \mathbf{e}_{\mathrm{n}}\right): \mathbf{W} \mathrm{d} \Omega=\frac{S_{d-1}}{d} \mathbf{W}, \\
& \int_{\mathbb{S}^{d-1}} \mathbf{e}_{\mathrm{n}}\left(\mathbf{e}_{\mathrm{n}} \cdot \mathbf{e}_{\mathrm{n}}\right) \mathrm{d} \Omega=\int_{\mathbb{S}^{d-1}} \mathbf{e}_{\mathrm{n}} \mathrm{d} \Omega=0 .
\end{aligned}
$$

Combining the above, we have

$$
I_{1}=-\frac{16 S_{d-1}}{M^{3} d}(\mathbf{V}-A \mathbf{Q}) \Phi_{1}+O\left(m^{3 / 10}\right)
$$

Multiplying by $C_{m}$ of (B.4) gives the drift coefficient

$$
-\frac{\gamma}{M}(\mathbf{V}-A \mathbf{Q})=\lim _{m \rightarrow 0} C_{m} I_{1}=-\frac{4 \lambda R^{d-1} S_{d-1}}{M d} \Phi_{1}(\mathbf{V}-A \mathbf{Q})
$$

In the case of $f(v)=Z^{-1} \exp \left(-\frac{\beta v^{2}}{2}\right)$ with $Z=\frac{\sqrt{2 \pi}}{\sqrt{\beta}}$, we have $\Phi_{1}=\frac{1}{\sqrt{2 \beta \pi}}$ and hence

$$
\gamma=\frac{2 \sqrt{2} \lambda R^{d-1} S_{d-1}}{\sqrt{\pi \beta} d} .
$$

\section{B.2. Stochastic limit}

Combining the expansion of $L_{m}$ in (B.3), with the expressions for $I_{1}$ and $I_{2}$ (B.6) and (B.7) and with the bound on the remainder (B.5), we have

$$
L_{m} \psi(\mathbf{Q}, \mathbf{V})=\mathbf{V} \cdot \nabla_{Q} \psi(\mathbf{Q}, \mathbf{V})-M^{-1} \gamma(\mathbf{V}-A \mathbf{Q}) \cdot \nabla_{V} \psi(\mathbf{Q}, \mathbf{V})+\frac{1}{2} D_{V} \psi(\mathbf{Q}, \mathbf{V})+O\left(m^{3 / 10}\right)
$$

We thus have a generator $L_{m}$ that in the limit $m \rightarrow 0$ converges in the sense of (B.2) to the generator of the nonequilibrium Langevin dynamics (2.21), where $\gamma$ is given in (B.7) and $\sigma=M \sqrt{D}$ is defined using (B.6), in agreement with (2.22). Thus, we use Lemma B.2 to conclude the convergence of the Markov process as stated in Lemma B.1. 


\section{Appendix C. Fast COllisions CANnot leAd to ReCOllisions}

We show in this section that in the mechanical process until the stopping time (A.3), any bath atom that undergoes a fast collision (recall Definition A.1) cannot recollide with the large particle and that the bath atom cannot have previously collided with the large particle. This shows the fast collisions experienced by the large particle have rate $r_{m}$ in (A.4). This observation is necessary when coupling the Markov approximation to the mechanical process, which we do in Appendix D.

Lemma C.1. For a given trajectory $\left(\mathbf{Q}_{m}, \mathbf{V}_{m}\right)$, suppose that the large particle experiences a fast collision with a bath atom at time $t_{1} \in\left[0, \tau_{m}\right)$. Then there are no other collisions between this atom and the large particle in the time interval $\left[0, \tau_{m}\right)$, where $\tau_{m}$ denotes the stopping time (A.3).

Proof. We recall that the relative velocity of the bath atom is $\overline{\mathbf{v}}=\mathbf{v}-$ Aq. By the choice of bath dynamics (2.20), the relative velocity only changes by colliding with the large particle whereas the velocity $\mathbf{v}$ changes with time according to (2.18). We consider a fast collision,

$$
\bar{v}_{\mathrm{n}}>c_{m}=m^{-1 / 5} \text {. }
$$

We write the collision rule (2.7) in terms of relative velocity before making use of the bound on particle position and velocity in (A.3) (note that since the collision occurs on the particle's surface, $\mathbf{q}\left(t_{1}\right)$ can be bounded in terms of $\left.\mathbf{Q}\left(t_{1}\right)\right)$. We then have the following bound on the relative normal velocity of the bath atom after the collision

$$
\begin{aligned}
\bar{v}_{\mathrm{n}}^{\prime} & =-\frac{M-m}{M+m} \bar{v}_{\mathrm{n}}+\frac{2 M}{M+m} V_{\mathrm{n}}-\frac{2 M}{M+m}\left(A \mathbf{q}\left(t_{1}\right)\right)_{\mathrm{n}} \\
& \leq-\left(\frac{M-m}{M+m}\right) c_{m}+\frac{2 M}{M+m}\left(\frac{c_{m}}{8}+R\|A\|\right) \\
& \leq-\frac{2}{3} c_{m} .
\end{aligned}
$$

The last line holds for $m$ sufficiently small. This shows that after colliding, the bath atom moves away from the particle with a large velocity.

We let $\mathbf{e}_{\mathrm{n}, 1}$ denote the normal vector for the fast collision at time $t_{1}$ and look at the future velocity of the bath atom, $\mathbf{v}(t)=A \mathbf{q}(t)+\overline{\mathbf{v}}^{\prime}(t)$ for $t \in\left[t_{1}, \tau_{m}\right)$. From the above computation we see that the relative velocity of the bath atom is pointed away from the large particle in the $\mathbf{e}_{\mathrm{n}, 1}$-direction. Due to the bound on the position of the large particle in (A.3), any recollision with the bath atom must occur in the region ||$A|||\mathbf{Q}| \leq c_{m} / 8$; however, whenever the bath atom is in this region, its velocity in the $\mathbf{e}_{\mathrm{n}, 1}$-direction satisfies $\mathbf{v}(t) \cdot \mathbf{e}_{\mathrm{n}, 1} \leq\left(c_{m} / 8-2 c_{m} / 3\right) \leq-\frac{c_{m}}{2}$. The large particle's velocity is bounded by $c_{m} / 8$ and is thus too low to overtake the bath atom. Therefore, there cannot be a recollision before time $\tau_{m}$. Likewise, before the collision, the velocity in the $\mathbf{e}_{\mathrm{n}, 1}$ direction satisfied $\mathbf{v}(t) \cdot \mathbf{e}_{\mathrm{n}, 1} \geq \frac{3 c_{m}}{4}$, which is faster than the large particle's speed which is bounded by $c_{m} / 8$, so it is impossible that there were previous collisions before time $t=0$.

\section{Appendix D. Coupling the mechanical process to the Markov approximation}

In this section, we couple the Markov process $\left(\widetilde{\mathbf{Q}}_{m}, \widetilde{\mathbf{V}}_{m}\right)$ with the rate (A.7) to the mechanical process $\left(\mathbf{Q}_{m}, \mathbf{V}_{m}\right)$, defined in Section 2.1. That is, for each realization of the mechanical process, we associate a set of realizations of the Markov process that are, with high probability, close in the $L^{\infty}$-norm. For the coupling, we prove below that

$$
\left(\widetilde{\mathbf{Q}}_{m}, \widetilde{\mathbf{V}}_{m}\right)_{\{0 \leq t \leq T\}}-\left(\mathbf{Q}_{m}, \mathbf{V}_{m}\right)_{\{0 \leq t \leq T\}} \stackrel{p}{\longrightarrow} 0 \text { as } m \rightarrow 0 .
$$

As in [7], we define a stopping time when the processes first differ by $\varepsilon>0$ and bound the total effect of the velocity jumps not shared between the two processes to show that in the limit $m \rightarrow 0$ the stopping time is greater than or equal to $T$ with probability 1. 
As described below, for the majority of the velocity jumps in the mechanical process caused by fast collisions, we subject the Markov process to the same velocity jump. The construction of the Markov process differs slightly from that in [7]. Here, we couple jumps in the velocity rather than collisions with bath atoms, which makes the argument simpler. This simplification is made possible by the additional assumption that $f(x)$ is decreasing, which we have assumed in our case in order to properly handle the fact that the bath atom velocity distribution depends on position.

\section{D.1. Coupling and convergence}

We construct the coupling up to the stopping time $\tau_{m}$ for the mechanical process (A.3), and extend the definition of the Markov process up to time $T$, if necessary. Let $I_{\text {mech }}=\left\{t_{1}, t_{2}, \ldots\right\}$ denote the set of all times up to $\tau_{m}$ at which the large particle in the mechanical process experiences a collision. This set is shown to be almost surely finite for any $m$ in Appendix E. We define $\mathbf{v}_{\mathrm{n}, i}=\mathbf{v}_{\mathrm{n}}\left(t_{i}\right), \overline{\mathbf{v}}_{\mathrm{n}, i}=\overline{\mathbf{v}}_{\mathrm{n}}\left(t_{i}\right), \mathbf{V}_{\mathrm{n}, i}=\mathbf{V}_{\mathrm{n}}\left(t_{i}\right)$, $\mathbf{e}_{\mathrm{n}, i}=\mathbf{e}_{\mathrm{n}}\left(t_{i}\right)$, etc. We let $I_{\text {slow }}=\left\{t_{i} \in I_{\text {mech }}:\left|\overline{\mathbf{v}}_{\mathrm{n}, i}\right|<c_{m}\right\}$ denote the set of jump times due to slow collisions and $I_{\text {fast }}=I_{\text {mech }} \backslash I_{\text {slow }}$ denote the set of jump times due to fast collisions. These sets of jump times are random variables since the initial condition is random.

For a given trajectory of the mechanical process $\left(\mathbf{Q}_{m}, \mathbf{V}_{m}\right)$, we define $\left(\widetilde{\mathbf{Q}}_{m}(0), \widetilde{\mathbf{V}}_{m}(0)\right)=\left(\mathbf{Q}_{m}(0), \mathbf{V}_{m}(0)\right)$, and for most of the fast collisions of the mechanical process we subject the Markov process to the same jump in velocity that the mechanical process undergoes. We selectively accept some subset of the fast collisions in the time interval $\left[0, \tau_{m}\right)$ and add additional jumps in the velocity to ensure that the jumps of the Markov process have the rate $\hat{r}_{m}\left(\widehat{V}, \mathbf{e}_{\mathrm{n}}, \widetilde{\mathbf{Q}}_{m}, \widetilde{\mathbf{V}}_{m}\right)$ defined in (A.7). (Recall that the Markov process has no slow collisions). More precisely, for every $t_{i} \in I_{\text {fast }}$, we choose to apply a jump with velocity change $\widehat{V}_{i} \mathbf{e}_{\mathrm{n}, i}$ to the Markov process with probability

$$
p_{\text {keep }}\left(\widehat{V}_{i}, \mathbf{e}_{\mathrm{n}, i}, \widetilde{\mathbf{Q}}_{m}, \widetilde{\mathbf{V}}_{m}, \mathbf{Q}_{m}, \mathbf{V}_{m}\right)=\min \left(\frac{\hat{r}_{m}\left(\widehat{V}_{i}, \mathbf{e}_{\mathrm{n}, i}, \widetilde{\mathbf{Q}}_{m}, \widetilde{\mathbf{V}}_{m}\right)}{\hat{r}_{m}\left(\widehat{V}_{i}, \mathbf{e}_{\mathrm{n}, i}, \mathbf{Q}_{m}, \mathbf{V}_{m}\right)}, 1\right) .
$$

For $t \in\left[0, \tau_{m}\right)$, we add additional fast collisions to the Markov process with the Poisson rate

$$
r_{\text {add }}\left(\widehat{V}, \mathbf{e}_{\mathrm{n}}, \widetilde{\mathbf{Q}}_{m}, \widetilde{\mathbf{V}}_{m}, \mathbf{Q}_{m}, \mathbf{V}_{m}\right)=\max \left(\hat{r}_{m}\left(\widehat{V}, \mathbf{e}_{\mathrm{n}}, \widetilde{\mathbf{Q}}_{m}, \widetilde{\mathbf{V}}_{m}\right)-\hat{r}_{m}\left(\widehat{V}, \mathbf{e}_{\mathrm{n}}, \mathbf{Q}_{m}, \mathbf{V}_{m}\right), 0\right) .
$$

After accepting collisions with probability (D.1), and adding new collisions with rate (D.2), a short calculation shows that the rate function for the Markov process in the time interval $\left[0, \tau_{m}\right)$ is $\hat{r}_{m}\left(\widehat{V}, \mathbf{e}_{\mathrm{n}}, \widetilde{\mathbf{Q}}_{m}, \widetilde{\mathbf{V}}_{m}\right)$. If $\tau_{m}<T$, we extend the Markov process to $[0, T]$ by adding additional jumps with rate $\hat{r}_{m}\left(\widehat{V}, \mathbf{e}_{n}, \widetilde{\mathbf{Q}}_{m}, \widetilde{\mathbf{V}}_{m}\right)$.

We let $I_{\text {Mark }}$ denote the set of all jump times of the Markov process, $I_{\text {rem }} \subset I_{\text {fast }}$ denote the set of jump times of the mechanical process that were not chosen for the Markov process, and $I_{\text {add }} \subset I_{\text {Mark }}$ the set of additional times at which jumps were added to the Markov process (in the whole interval $[0, T]$ ). From the construction above, we have that

$$
I_{\text {Mark }}=\left(I_{\text {fast }} \backslash I_{\text {rem }}\right) \cup I_{\text {add }} .
$$

We note that the sets of jump times $I_{\text {mech }}, I_{\text {Mark }}, I_{\text {slow }}, I_{\text {rem }}$, and $I_{\text {add }}$ are random variables.

Since the chosen realization of the mechanical process may not be defined on the full time interval of interest $[0, T]$, we make the convention that

$$
\sup _{s \in[0, t]}\left|\widetilde{\mathbf{V}}_{m}(s)-\mathbf{V}_{m}(s)\right|=\infty \quad \text { if } \tau_{m}<t .
$$

In particular, part of the proof of convergence will be the fact that $\lim _{m \rightarrow 0} \mathbb{P}\left(\tau_{m}=T\right)=1$. We then show the following convergence in probability result.

Lemma D.1. For all $T>0$,

$$
\left(\widetilde{\mathbf{Q}}_{m}, \widetilde{\mathbf{V}}_{m}\right)_{\{0 \leq t \leq T\}}-\left(\mathbf{Q}_{m}, \mathbf{V}_{m}\right)_{\{0 \leq t \leq T\}} \stackrel{p}{\longrightarrow} 0 \text { as } m \rightarrow 0,
$$


where the convergence in probability is with respect to the $L^{\infty}([0, T])$-norm. Equivalently, for all $T>0$ and any $\varepsilon>0$,

$$
\lim _{m \rightarrow 0} \mathbb{P}\left(\sup _{t \in[0, T]}\left|\widetilde{\mathbf{Q}}_{m}(t)-\mathbf{Q}_{m}(t)\right|+\left|\widetilde{\mathbf{V}}_{m}(t)-\mathbf{V}_{m}(t)\right| \geq \varepsilon\right)=0 .
$$

To show Lemma D.1, we prove the following lemma which says that the interval of convergence can be extended by a finite time step.

Lemma D.2. If $t_{0} \geq 0$ is such that

$$
\lim _{m \rightarrow 0} \mathbb{P}\left(\sup _{t \in\left[0, t_{0}\right]}\left|\widetilde{\mathbf{Q}}_{m}(t)-\mathbf{Q}_{m}(t)\right|+\left|\widetilde{\mathbf{V}}_{m}(t)-\mathbf{V}_{m}(t)\right| \geq \varepsilon\right)=0
$$

for all $\varepsilon>0$, we then have

$$
\lim _{m \rightarrow 0} \mathbb{P}\left(\sup _{t \in\left[0, t_{0}+z\right]}\left|\widetilde{\mathbf{Q}}_{m}(t)-\mathbf{Q}_{m}(t)\right|+\left|\widetilde{\mathbf{V}}_{m}(t)-\mathbf{V}_{m}(t)\right| \geq \varepsilon\right)=0
$$

for all $\varepsilon>0$ where $z=\min \left(M\left(192 \lambda R^{d-1} S_{d-1} \Phi_{1}\right)^{-1}, \frac{1}{2(1+\|A\|)}\right)$.

Since the initial conditions for the mechanical process and Markov process are the same, the hypothesis of Lemma D.2 holds for $t_{0}=0$. Thus, Lemma D.1 follows immediately from Lemma D.2, and we are left with proving Lemma D.2. The computation justifying the particular choice of $z$ is performed in Appendix D.3.4.

\section{D.2. Error decomposition}

We prove Lemma D.2 by splitting the error in the velocity into two contributions and bounding them individually. We fix $\varepsilon>0$ and $t_{0}>0$, and in the following we denote by $I_{\text {mech }}^{\prime}=I_{\text {mech }} \cap\left[t_{0}, t_{0}+z\right]$, $I_{\text {Mark }}^{\prime}=I_{\text {Mark }} \cap\left[t_{0}, t_{0}+z\right]$, and likewise for $I_{\text {slow }}^{\prime}, I_{\text {rem }}^{\prime}$, and $I_{\text {add }}^{\prime}$. For times $t \in\left[t_{0}, \tau_{m}\right)$, the error in velocity between the Markov and mechanical processes is

$$
\left|\mathbf{V}_{m}(t)-\widetilde{\mathbf{V}}_{m}(t)\right|=\left|\mathbf{V}_{m}\left(t_{0}\right)+\sum_{t_{i} \in I_{\text {mech }}^{\prime}} \widehat{V}_{i} \mathbf{e}_{\mathrm{n}, i} \mathbb{1}_{\left[t_{0}, t\right]}\left(t_{i}\right)-\widetilde{\mathbf{V}}_{m}\left(t_{0}\right)-\sum_{t_{i} \in I_{\text {Mark }}^{\prime}} \widehat{V}_{i} \mathbf{e}_{\mathrm{n}, i} \mathbb{1}_{\left[t_{0}, t\right]}\left(t_{i}\right)\right|
$$

where we recall that $t_{i}$ is the time that the jump $\widehat{V}_{i} \mathbf{e}_{\mathrm{n}, i}$ occurs and where $\mathbb{1}_{S}$ denotes the characteristic function of the set $S$.

We split the error into two sources. The first source of error is the change in velocity due to slow collisions of the mechanical process, since the Markov process does not include any slow collisions. We denote this contribution in the interval $\left[t_{0}, t\right]$ by

$$
W_{\text {slow }}(t)=\left|\sum_{t_{i} \in I_{\text {slow }}^{\prime}} \widehat{V}_{i} \mathbf{e}_{\mathrm{n}, i} \mathbb{1}_{\left[t_{0}, t\right]}\left(t_{i}\right)\right| .
$$

The second source of error is the change in velocity due to the jumps that we added and removed when coupling the Markov process to the mechanical process. We define

$$
W_{\mathrm{ex}}(t)=\sum_{t_{i} \in I_{\mathrm{rem}}^{\prime}} \widehat{V}_{i} \mathbb{1}_{\left[t_{0}, t\right]}\left(t_{i}\right)+\sum_{t_{i} \in I_{\text {add }}^{\prime}} \widehat{V}_{i} \mathbb{1}_{\left[t_{0}, t\right]}\left(t_{i}\right) .
$$

By definition, $W_{\text {ex }}(t) \geq 0$ for all $t$ (recall that $\widehat{V} \geq 0$ ). The error terms $W_{\text {slow }}$ and $W_{\text {ex }}$ are random variables. 
We decompose the error in the velocity as

$$
\begin{aligned}
\left|\mathbf{V}_{m}(t)-\widetilde{\mathbf{V}}_{m}(t)\right|= & \left|\mathbf{V}_{m}\left(t_{0}\right)+\sum_{t_{i} \in I_{\text {mech }}^{\prime}} \widehat{V}_{i} \mathbf{e}_{\mathrm{n}, i} \mathbb{1}_{\left[t_{0}, t\right]}\left(t_{i}\right)-\widetilde{\mathbf{V}}_{m}\left(t_{0}\right)-\sum_{t_{i} \in I_{\text {Mark }}^{\prime}} \widehat{V}_{i} \mathbf{e}_{\mathrm{n}, i} \mathbb{1}_{\left[t_{0}, t\right]}\left(t_{i}\right)\right| \\
\leq & \left|\mathbf{V}_{m}\left(t_{0}\right)-\widetilde{\mathbf{V}}_{m}\left(t_{0}\right)\right|+\left|\sum_{t_{i} \in I_{\text {slow }}^{\prime}} \widehat{V}_{i} \mathbf{e}_{\mathrm{n}, i} \mathbb{1}_{\left[t_{0}, t\right]}\left(t_{i}\right)\right| \\
& +\sum_{t_{i} \in I_{\text {add }}^{\prime}}\left|\widehat{V}_{i} \mathbf{e}_{\mathrm{n}, i} \mathbb{1}_{\left[t_{0}, t\right]}\left(t_{i}\right)\right|+\sum_{t_{i} \in I_{\text {rem }}^{\prime}}\left|\widehat{V}_{i} \mathbf{e}_{\mathrm{n}, i} \mathbb{1}_{\left[t_{0}, t\right]}\left(t_{i}\right)\right| \\
= & \left|\mathbf{V}_{m}\left(t_{0}\right)-\widetilde{\mathbf{V}}_{m}\left(t_{0}\right)\right|+W_{\text {slow }}(t)+W_{\text {ex }}(t) .
\end{aligned}
$$

We fix $\varepsilon>0$ and, as in [7], define the stopping time

$$
\tau_{m}^{*}=\min \left(\inf _{t \in\left[t_{0}, \tau_{m}\right)}\left\{t:\left|\widetilde{\mathbf{V}}_{m}(t)-\mathbf{V}_{m}(t)\right| \geq \varepsilon / 2\right\}, t_{0}+z\right) .
$$

The use of a stopping time gives us a bound on how close the mechanical and Markov processes are, which allows us to then bound the difference in jumps each process experiences. Provided that we have $\left|\widetilde{\mathbf{Q}}_{m}\left(t_{0}\right)-\mathbf{Q}_{m}\left(t_{0}\right)\right| \leq$ $\varepsilon / 4$, which we can assume from the hypothesis in Lemma D.2, we have that

$$
\sup _{t \in\left[t_{0}, \tau_{m}^{*}\right)}\left|\widetilde{\mathbf{Q}}_{m}(t)-\mathbf{Q}_{m}(t)\right| \leq\left|\widetilde{\mathbf{Q}}_{m}\left(t_{0}\right)-\mathbf{Q}_{m}\left(t_{0}\right)\right|+z \sup _{t \in\left[t_{0}, \tau_{m}^{*}\right)}\left|\widetilde{\mathbf{V}}_{m}(t)-\mathbf{V}_{m}(t)\right| \leq \varepsilon / 2,
$$

where we recall $z \leq \frac{1}{2(1+\|A\|)}$. Thus, we have the relations

$$
\begin{aligned}
& \sup _{t \in\left[t_{0}, \tau_{m}^{*}\right)}\left|\widetilde{\mathbf{Q}}_{m}(t)-\mathbf{Q}_{m}(t)\right|+\left|\widetilde{\mathbf{V}}_{m}(t)-\mathbf{V}_{m}(t)\right| \leq \varepsilon, \\
& \sup _{t \in\left[t_{0}, \tau_{m}^{*}\right)}\|A\|\left|\widetilde{\mathbf{Q}}_{m}(t)-\mathbf{Q}_{m}(t)\right|+\left|\widetilde{\mathbf{V}}_{m}(t)-\mathbf{V}_{m}(t)\right| \leq \varepsilon .
\end{aligned}
$$

From the above arguments, we may show Lemma D.2 by showing that

$$
\lim _{m \rightarrow 0} \mathbb{P}\left(\left\{\tau_{m}^{*}<t_{0}+z\right\}\right)=0 .
$$

It is difficult to estimate a priori the probability $\mathbb{P}\left(\tau_{m}<t_{0}+z\right)$, which appears indirectly in the estimate of $\mathbb{P}\left(\tau_{m}^{*}<t_{0}+z\right)$ through the definition of $\tau_{m}^{*}$ (D.6). To aid in the estimate we define the set of trajectories where the Markov process remains small compared to $c_{m}$ is

$$
G_{m}=\left\{\sup _{0 \leq t \leq T}\left(\|A\|\left|\widetilde{\mathbf{Q}}_{m}(t)\right|+\left|\widetilde{\mathbf{V}}_{m}(t)\right|\right) \leq c_{m} / 16\right\} .
$$

From the convergence of the Markov process in Lemma B.1, we have that

$$
\lim _{m \rightarrow 0} \mathbb{P}\left(G_{m}\right)=1 \text {. }
$$

By the triangle inequality, we see that for trajectories belonging to $G_{m}, \tau_{m}^{*}<t_{0}+z \Rightarrow\left|\widetilde{\mathbf{V}}_{m}\left(\tau^{*}\right)-\mathbf{V}_{m}\left(\tau^{*}\right)\right| \geq$ $\varepsilon / 2$. Thus, in order to have (D.8), it is sufficient to show that

$$
\lim _{m \rightarrow 0} \mathbb{P}\left(\left\{\tau_{m}^{*}<t_{0}+z\right\} \cap G_{m}\right)=0 .
$$


To show (D.11), we use (D.5) to break apart the terms:

$$
\begin{aligned}
\left\{\tau_{m}^{*}<t_{0}+z\right\} \cap G_{m} \subset\left\{\left\{W_{\text {slow }}\left(\tau_{m}^{*}\right) \geq \varepsilon / 6\right\} \cap G_{m}\right\} \cup\left\{\left\{W_{\text {ex }}\left(\tau_{m}^{*}\right) \geq \varepsilon / 6\right\} \cap G_{m}\right\} \\
\cup\left\{\left|\widetilde{\mathbf{V}}_{m}\left(t_{0}\right)-\mathbf{V}_{m}\left(t_{0}\right)\right| \geq \varepsilon / 6\right\} .
\end{aligned}
$$

In terms of probability, we have

$$
\begin{aligned}
\mathbb{P}\left(\left\{\tau_{m}^{*}<t_{0}+z\right\} \cap G_{m}\right) \leq & \mathbb{P}\left(\left\{W_{\text {slow }}\left(\tau_{m}^{*}\right) \geq \varepsilon / 6\right\} \cap G_{m}\right)+\mathbb{P}\left(\left\{W_{\text {ex }}\left(\tau_{m}^{*}\right) \geq \varepsilon / 6\right\} \cap G_{m}\right) \\
& +\mathbb{P}\left(\left|\widetilde{\mathbf{V}}_{m}\left(t_{0}\right)-\mathbf{V}_{m}\left(t_{0}\right)\right| \geq \varepsilon / 6\right) .
\end{aligned}
$$

By the hypothesis of Lemma D.2,

$$
\lim _{m \rightarrow 0} \mathbb{P}\left(\left\{\left|\tilde{\mathbf{V}}_{m}\left(t_{0}\right)-\mathbf{V}_{m}\left(t_{0}\right)\right| \geq \varepsilon / 6\right\}\right)=0 .
$$

We prove convergence of the other two terms in the following sections.

\section{D.3. Different collision rates}

Since we restrict our attention to times $t<\tau_{m}$, Appendix $\mathrm{C}$ shows that the rate of jumps due to fast collisions in the mechanical process is given by (A.7). We now estimate

$$
W_{\mathrm{ex}}\left(\tau_{m}^{*}\right)=\sum_{t_{i} \in I_{\mathrm{rem}}^{\prime}} \widehat{V}_{i} \mathbb{1}_{\left[t_{0}, \tau_{m}^{*}\right]}\left(t_{i}\right)+\sum_{t_{i} \in I_{\mathrm{add}}^{\prime}} \widehat{V}_{i} \mathbb{1}_{\left[t_{0}, \tau_{m}^{*}\right]}\left(t_{i}\right)
$$

which bounds the total effect of the jumps that were added and removed in the coupling process using the expressions in (D.1) and (D.2). These jumps have the rate function

$$
r_{\mathrm{ex}}\left(\widehat{V}, \mathbf{e}_{\mathrm{n}}, \widetilde{\mathbf{Q}}_{m}, \widetilde{\mathbf{V}}_{m}, \mathbf{Q}_{m}, \mathbf{V}_{m}\right)=\left|\hat{r}_{m}\left(\widehat{V}, \mathbf{e}_{\mathrm{n}}, \widetilde{\mathbf{Q}}_{m}, \tilde{\mathbf{V}}_{m}\right)-\hat{r}_{m}\left(\widehat{V}, \mathbf{e}_{\mathrm{n}}, \mathbf{Q}_{m}, \mathbf{V}_{m}\right)\right|
$$

The goal of this subsection is to show the following.

Lemma D.3. Fix $\varepsilon>0$, and let $\tau_{m}^{*}$ be given by (D.6). Let $G_{m}$ be given by (D.9), and let $W_{\mathrm{ex}}$ be given by (D.4). We have that

$$
\lim _{m \rightarrow 0} \mathbb{P}\left(\left\{W_{\mathrm{ex}}\left(\tau_{m}^{*}\right) \geq \varepsilon / 6\right\} \cap G_{m}\right)=0 .
$$

Proof. In the following, we bound the error $W_{\mathrm{ex}}$ in terms of a pair of compound Poisson processes, whose definition we recall below. The error $W_{\text {ex }}$ is not a compound Poisson process itself, due to the dependence of $r_{\mathrm{ex}}$ on $\widetilde{\mathbf{Q}}_{m}, \widetilde{\mathbf{V}}_{m}, \mathbf{Q}_{m}, \mathbf{V}_{m}$, and $\mathbf{e}_{\mathrm{n}}$, but we build a compound Poisson process from realizations of $W_{\mathrm{ex}}$, by increasing the size of certain jumps as well as adding additional jumps as detailed below.

\section{D.3.1. Compound Poisson Processes}

We begin by recalling a few properties of compound Poisson processes before proving our bound on $W_{\text {ex }}$. (Definitions may be found for instance in [5]).

Definition D.4. A compound Poisson process $J$ is a stochastic process defined in terms of its rate function $r(\check{V})$ by

$$
J(t)=\sum_{i=1}^{N(t)} \check{V}_{i}
$$

where $N(t)$ is a Poisson process with rate $\Lambda:=\int_{\mathbb{R}} r(\check{V}) d \check{V}$ that is independent from the jumps $\left\{\check{V}_{i}\right\}_{i=1}^{N}$ which are independent, identically distributed random variables with probability density function $\Lambda^{-1} r(\check{V})$. 
The expected value of a compound Poisson process satisfies

$$
\mathbb{E}(J(t))=\mathbb{E}(N(t)) \mathbb{E}(\check{V})=t \int_{\mathbb{R}} \check{V} r(\check{V}) d \check{V} \mathrm{~d} t .
$$

Using the independence of $\left\{\check{V}_{i}\right\}_{1 \leq i \leq N_{\text {ex }}}$ and $N_{\text {ex }}$, we can simplify the variance

$$
\begin{aligned}
\mathbb{E}\left((J(t)-\mathbb{E}(J(t)))^{2}\right) & =\mathbb{E}\left(\left(\sum_{i=1}^{N(t)} \check{V}_{i}-\mathbb{E}(J(t))\right)^{2}\right) \\
& =\mathbb{E}\left(\left(\sum_{i=1}^{N(t)} \check{V}_{i}-\mathbb{E}(N(t)) \mathbb{E}(\check{V})\right)^{2}\right) \\
& =\mathbb{E}\left(\left(\sum_{i=1}^{N(t)}\left(\check{V}_{i}-\mathbb{E}(\check{V})\right)+[N(t)-\mathbb{E}(N(t))] \mathbb{E}(\check{V})\right)^{2}\right) \\
& =\mathbb{E}\left(\sum_{i=1}^{N(t)}\left(\check{V}_{i}-\mathbb{E}(\check{V})\right)^{2}\right)+\mathbb{E}\left(\left(N(t)-\mathbb{E}(N(t))^{2}\right) \mathbb{E}(\check{V})^{2}\right. \\
& =\mathbb{E}(N(t)) \mathbb{E}\left([\check{V}-\mathbb{E}(\check{V})]^{2}\right)+\mathbb{E}(N(t)) \mathbb{E}(\check{V})^{2} \\
& =\mathbb{E}(N(t)) \mathbb{E}\left(\check{V}^{2}\right) \\
& =t \int_{\mathbb{R}} \check{V}^{2} r(\check{V}) d \check{V} .
\end{aligned}
$$

To go from the third to the fourth line, we have used independence of $N(t)$ and $\check{V}_{i}$, and to go from the fourth to the fifth we have used the independence of the jumps $\left\{\breve{V}_{i}\right\}_{i=1}^{N(t)}$ and the following property of the variance of exponential distributions:

$$
\mathbb{E}\left(\left(N(t)-\mathbb{E}(N(t))^{2}\right)=\mathbb{E}(N(t)) .\right.
$$

\section{D.3.2. Building the auxiliary process}

Since there are Heaviside functions within the definition of $\hat{r}_{m}$, we consider three regions: one where both rate functions are nonzero, a second where one rate function is identically zero, and a third where they are both identically zero. For $t \in\left[t_{0}, \tau_{m}^{*}\right)$, we define

$$
\begin{aligned}
& v_{1}=\frac{2 m}{M+m} \min \left(\left(c_{m}-\widetilde{V}_{\mathrm{n}}+(A \widetilde{\mathbf{q}})_{\mathrm{n}}\right),\left(c_{m}-V_{\mathrm{n}}+(A \mathbf{q})_{\mathrm{n}}\right)\right) \\
& v_{2}=\frac{2 m}{M+m} \max \left(\left(c_{m}-\widetilde{V}_{\mathrm{n}}+(A \widetilde{\mathbf{q}})_{\mathrm{n}}\right),\left(c_{m}-V_{\mathrm{n}}+(A \mathbf{q})_{\mathrm{n}}\right)\right)
\end{aligned}
$$

where we recall that $\mathbf{q}=\mathbf{Q}_{m}-R \mathbf{e}_{\mathrm{n}}$ and $\widetilde{\mathbf{q}}=\widetilde{\mathbf{Q}}_{m}-R \mathbf{e}_{\mathrm{n}}$ are the locations of the collision on the surface of the respective atoms. Note that $\frac{2 m}{M+m} \frac{7 c_{m}}{8} \leq v_{1} \leq v_{2} \leq \frac{2 m}{M+m} \frac{9 c_{m}}{8}$ from (D.9) and (A.3), and $v_{2}-v_{1} \leq \frac{2 m}{M+m} \varepsilon$ from (D.7).

In the range $v_{1} \leq \widehat{V} \leq v_{2}$, we can directly bound $r_{\text {ex }}$ using the monotonicity of $f$

$$
\begin{aligned}
r_{\mathrm{ex}}\left(\widehat{V}, \mathbf{e}_{\mathrm{n}}, \widetilde{\mathbf{Q}}_{m}, \widetilde{\mathbf{V}}_{m}, \mathbf{Q}_{m}, \mathbf{V}_{m}\right) & =C_{m} m^{-5 / 2} \widehat{V} f_{m}^{1}\left(\frac{M+m}{2 m}\left(\widehat{V}-v_{1}\right)+c_{m}\right) \\
& \leq C_{m} m^{-5 / 2} \widehat{V} f_{m}^{1}\left(\frac{M+m}{2 m} \widehat{V}-\frac{c_{m}}{8}\right)
\end{aligned}
$$


where

$$
C_{m}=\lambda R^{d-1}\left(\frac{M+m}{2}\right)^{2}
$$

We integrate out $\mathbf{e}_{\mathrm{n}}$, giving the factor $S_{d-1}$, and define the rate

$$
r_{1}(\widehat{V})=C_{m} S_{d-1} m^{-5 / 2} \widehat{V} f_{m}^{1}\left(\frac{M+m}{2 m} \widehat{V}-\frac{c_{m}}{8}\right) .
$$

To construct the Poisson process, we add additional jumps in $\widehat{V}$ with the (positive) rate

$$
r_{1}(\widehat{V})-\int_{\mathbb{S}^{d-1}} r_{\mathrm{ex}}\left(\widehat{V}, \mathbf{e}_{\mathrm{n}}, \widetilde{\mathbf{Q}}_{m}, \widetilde{\mathbf{V}}_{m}, \mathbf{Q}_{m}, \mathbf{V}_{m}\right) \mathrm{d} \Omega .
$$

For $v_{2} \leq \widehat{V}<\infty$, we have the following estimate:

$$
\begin{aligned}
r_{\mathrm{ex}}\left(\widehat{V}, \mathbf{e}_{\mathrm{n}}, \widetilde{\mathbf{Q}}_{m}, \widetilde{\mathbf{V}}_{m}, \mathbf{Q}_{m}, \mathbf{V}_{m}\right) \\
\quad=C_{m} m^{-5 / 2} \widehat{V}\left|f_{m}^{1}\left(\frac{M+m}{2 m} \widehat{V}+\widetilde{V}_{\mathrm{n}}-(A \widetilde{\mathbf{q}})_{\mathrm{n}}\right)-f_{m}^{1}\left(\frac{M+m}{2 m} \widehat{V}+V_{\mathrm{n}}-(A \mathbf{q})_{\mathrm{n}}\right)\right| \\
\quad=C_{m} m^{-5 / 2} \widehat{V}\left[f_{m}^{1}\left(\frac{M+m}{2 m}\left(\widehat{V}-v_{2}\right)+c_{m}\right)-f_{m}^{1}\left(\frac{M+m}{2 m}\left(\widehat{V}-v_{1}\right)+c_{m}\right)\right] \\
\quad \leq C_{m} m^{-5 / 2} \widehat{V}\left[f_{m}^{1}\left(\frac{M+m}{2 m}\left(\widehat{V}-v_{2}\right)+c_{m}\right)-f_{m}^{1}\left(\frac{M+m}{2 m}\left(\widehat{V}-v_{2}\right)+c_{m}+\varepsilon\right)\right]
\end{aligned}
$$

where the inequality uses the monotonicity of $f^{1}$. We cannot directly eliminate $v_{2}$ from the argument by relying on monotonicity arguments since we have a difference of $f$, so we shift by replacing jumps generated by the process corresponding to $r_{\text {ex }}$ by larger jumps. For any jump of size $v_{2} \leq \widehat{V}<\infty$, we replace it with a new, larger jump of size $\breve{V}=\widehat{V}+\frac{2 m}{M+m} \frac{9 c_{m}}{8}-v_{2}$. We also increase the rate of jumps by a factor of $\frac{\grave{V}}{\widehat{V}}$ and integrate out $\mathbf{e}_{\mathrm{n}}$, giving the rate

$$
r_{2}(\check{V})=C_{m} S_{d-1} m^{-5 / 2} \check{V}\left[f_{m}^{1}\left(\frac{M+m}{2 m} \check{V}-\frac{c_{m}}{8}\right)-f_{m}^{1}\left(\frac{M+m}{2 m} \check{V}-\frac{c_{m}}{8}+\varepsilon\right)\right] .
$$

The limits of the regions above, $v_{1}$ and $v_{2}$, also depend on $\left(\mathbf{Q}_{m}, \mathbf{V}_{m}\right)$ and $\left(\widetilde{\mathbf{Q}}_{m}, \widetilde{\mathbf{V}}_{m}\right)$. To remove this dependence we extend the interval of definition for the two rate functions defined above to $\frac{2 m}{M+m} \frac{7 c_{m}}{8} \leq \widehat{V} \leq \frac{2 m}{M+m} \frac{9 c_{m}}{8}$ for $r_{1}$ and to $\frac{2 m}{M+m} \frac{7 c_{m}}{8} \leq \check{V}<\infty$ for $r_{2}$. This can be done by adding additional jumps to the auxiliary Poisson processes above. All told, we have build realizations of two compound Poisson processes

$$
J_{m, k}=\sum_{i=1}^{N_{\mathrm{ex}, k}} \check{V}_{i} \mathbb{1}_{\left[t_{0}, t_{0}+z\right]}\left(t_{i}\right) \quad \text { for } k=1,2,
$$

with the respective rate functions

$$
\begin{aligned}
& \check{r}_{\mathrm{ex}, 1}(\check{V})=r_{1}(\check{V}) \mathbb{1}_{\left[\frac{7 c_{m}}{8}, \frac{9 c_{m}}{8}\right]}\left(\frac{M+m}{2 m} \check{V}\right), \\
& \check{r}_{\mathrm{ex}, 2}(\check{V})=r_{2}(\check{V}) \mathbb{1}_{\left[\frac{7 c_{m}}{8}, \infty\right)}\left(\frac{M+m}{2 m} \check{V}\right),
\end{aligned}
$$

where we have used the dummy variable $\check{V}$ for both processes and where we suppress dependence on time in the notation $J_{m, k}$. 
For trajectories in $G_{m}$, the Poisson processes $J_{m, 1}$ and $J_{m, 2}$ provide the bound

$$
W_{\mathrm{ex}}\left(\tau_{m}^{*}\right) \leq J_{m, 1}+J_{m, 2}
$$

Therefore,

$$
\mathbb{P}\left(\left\{W_{\mathrm{ex}}\left(\tau_{m}^{*}\right) \geq \varepsilon / 6\right\} \cap G_{m}\right) \leq \mathbb{P}\left(\left\{J_{m, 1} \geq \varepsilon / 12\right\} \cap G_{m}\right)+\mathbb{P}\left(\left\{J_{m, 2} \geq \varepsilon / 12\right\} \cap G_{m}\right) .
$$

To show that $\mathbb{P}\left(\left\{W_{\text {ex }}\left(\tau_{m}^{*}\right) \geq \varepsilon / 6\right\} \cap G_{m}\right) \rightarrow 0$ in (D.12), we show below that $\mathbb{P}\left(\left\{J_{m, k} \geq \varepsilon / 12\right\} \cap G_{m}\right) \rightarrow 0$ for $k=1,2$.

\section{D.3.3. Bounding $J_{m, 1}$}

Applying Markov's inequality, we have

$$
\mathbb{P}\left(\left\{J_{m, 1} \geq \varepsilon / 12\right\} \cap G_{m}\right) \leq \frac{12}{\varepsilon} \mathbb{E}_{m}\left(J_{m, 1}\right) .
$$

We make the substitution $x=\frac{M+m}{2 m^{1 / 2}} \check{V}-m^{1 / 2} \frac{c_{m}}{8}$ and bound the integrand on the finite interval to obtain the bound

$$
\begin{aligned}
\mathbb{E}_{m}\left(J_{m, 1}\right) & =\left(\left(t_{0}+z\right)-t_{0}\right) \int_{0}^{\infty} \check{V} r_{\mathrm{ex}, 1}(\check{V}) \mathrm{d} \check{V} \\
& =C_{m} S_{d-1} z m^{-5 / 2} \int_{\frac{2 m}{M+m} \frac{7 c_{m}}{8}}^{\frac{2 m}{M+m} \frac{9 c_{m}}{8}} \check{V}^{2} f_{m}^{1}\left(\frac{M+m}{2 m} \check{V}-\frac{c_{m}}{8}\right) d \check{V} \\
& =\frac{8 C_{m} S_{d-1} z m^{1 / 2}}{(M+m)^{3}} \int_{\frac{3}{4} m^{1 / 2} c_{m}}^{m^{1 / 2} c_{m}}\left(\frac{x}{m^{1 / 2}}+\frac{c_{m}}{8}\right)^{2} f^{1}(x) \mathrm{d} x \\
& \leq \frac{8 C_{m} S_{d-1} z m^{1 / 2}}{(M+m)^{3}}\left(\frac{9 c_{m}}{8}\right)^{2} \int_{0}^{\infty} f^{1}(x) \mathrm{d} x \\
& \leq \frac{81 \lambda R^{d-1} S_{d-1} z}{32(M+m)} m^{1 / 2} c_{m}^{2},
\end{aligned}
$$

where we recall the scaling of $f_{m}$ (A.5). We recall that $c_{m}=m^{-1 / 5}$ so that for any finite $z$ we have

$$
\lim _{m \rightarrow 0} \mathbb{E}_{m}\left(J_{m, 1}\right)=0
$$

Thus, we conclude

$$
\lim _{m \rightarrow 0} \mathbb{P}\left(\left\{J_{m, 1} \geq \varepsilon / 12\right\} \cap G_{m}\right)=0 .
$$




\section{D.3.4. Bounding $J_{m, 2}$}

We now wish to show

$$
\lim _{m \rightarrow 0} \mathbb{P}\left(\left\{J_{m, 2} \geq \varepsilon / 12\right\} \cap G_{m}\right)=0 .
$$

For this estimate, we first show the bound $\mathbb{E}_{m}\left(J_{m, 2}\right) \leq \varepsilon / 24$ before invoking Chebyshev's inequality. We have that

$$
\begin{aligned}
\mathbb{E}_{m}\left(J_{m, 2}\right)= & z \int_{0}^{\infty} \check{V}_{\mathrm{rex}, 2}(\check{V}) \mathrm{d} \check{V} \\
= & C_{m} S_{d-1} z m^{-5 / 2} \int_{\frac{2 m}{M+m} \frac{7 c_{m}}{8}}^{\infty} \check{V}^{2}\left[f_{m}^{1}\left(\frac{M+m}{2 m} \check{V}-\frac{c_{m}}{8}\right)-f_{m}^{1}\left(\frac{M+m}{2 m} \check{V}-\frac{c_{m}}{8}+\varepsilon\right)\right] d \check{V} \\
= & \frac{8 C_{m} S_{d-1}}{(M+m)^{3}} z m^{1 / 2}\left[\int_{\frac{3}{4} m^{1 / 2} c_{m}}^{\infty}\left(\frac{x}{m^{1 / 2}}+\frac{c_{m}}{8}\right)^{2} f(x) \mathrm{d} x\right. \\
& \left.-\int_{m^{1 / 2}\left(\frac{3}{4} c_{m}+\varepsilon\right)}^{\infty}\left(\frac{x}{m^{1 / 2}}+\frac{c_{m}}{8}-\varepsilon\right)^{2} f(x) \mathrm{d} x\right] \\
= & \frac{8 C_{m} S_{d-1}}{(M+m)^{3}} z m^{1 / 2}\left[\int_{0}^{\infty}\left(2 \varepsilon\left(\frac{x}{m^{1 / 2}}+\frac{c_{m}}{8}\right)-\varepsilon^{2}\right) f(x) \mathrm{d} x\right. \\
& \left.-\int_{0}^{\frac{3}{4} m^{1 / 2} c_{m}}\left(\frac{x}{m^{1 / 2}}+\frac{c_{m}}{8}\right)^{2} f(x) \mathrm{d} x+\int_{0}^{m^{1 / 2}\left(\frac{3}{4} c_{m}+\varepsilon\right)}\left(\frac{x}{m^{1 / 2}}+\frac{c_{m}}{8}-\varepsilon\right)^{2} f(x) \mathrm{d} x\right] \\
= & \frac{4 \lambda R^{d-1} S_{d-1}}{M} z \varepsilon \Phi_{1}+O\left(c_{m}^{2} m^{1 / 2}\right)
\end{aligned}
$$

where we made two changes of variables, $\frac{x}{m^{1 / 2}}=\frac{M+m}{2 m} \check{V}-\frac{c_{m}}{8}$ and $\frac{x}{m^{1 / 2}}=\frac{M+m}{2 m} \check{V}-\frac{c_{m}}{8}+\varepsilon$. We recall $\lim _{m \rightarrow 0} c_{m}^{2} m^{1 / 2}=0$, so that only the leading order term remains in the limit.

Now, by our choice of $z=\min \left(M\left(192 \lambda R^{d-1} S_{d-1} \Phi_{1}\right)^{-1}, \frac{1}{2(1+\|A\|)}\right)$ in Lemma D.2, we have

$$
\mathbb{E}_{m}\left(J_{m, 2}\right) \leq \varepsilon / 24
$$

whenever $m$ is sufficiently small. We later need a lower bound on the expected value, $\mathbb{E}_{m}\left(J_{m, 2}\right) \geq C \varepsilon$, where it is not necessary to precisely find the constant so long as $C$ does not depend on $\varepsilon$ or $m$. Such a bound follows from the above equations and the definition of $z$.

Applying Chebyshev's inequality and using (D.15), we have

$$
\begin{aligned}
\mathbb{P}\left(\left\{J_{m, 2} \geq \varepsilon / 12\right\} \cap G_{m}\right) & \leq \mathbb{P}\left(\left\{J_{m, 2} \geq \varepsilon / 12\right\}\right) \\
& \leq \mathbb{P}\left(\left\{J_{m, 2} \geq 2 \mathbb{E}_{m}\left(J_{m, 2}\right)\right\}\right) \\
& =\mathbb{P}\left(\left\{J_{m, 2}-\mathbb{E}_{m}\left(J_{m, 2}\right) \geq \mathbb{E}_{m}\left(J_{m, 2}\right)\right\}\right) \\
& \leq \frac{\mathbb{E}_{m}\left(\left(J_{m, 2}-\mathbb{E}_{m}\left(J_{m, 2}\right)\right)^{2}\right)}{\mathbb{E}_{m}\left(J_{m, 2}\right)^{2}} \\
& =\frac{\mathbb{E}_{m}\left(N_{\mathrm{ex}, 2}\right) \mathbb{E}_{m}\left(\check{V}^{2}\right)}{\mathbb{E}_{m}\left(J_{m, 2}\right)^{2}}
\end{aligned}
$$

where the final inequality follows from (D.13). 
We can then estimate $\mathbb{E}_{m}\left(N_{\mathrm{ex}, 2}\right) \mathbb{E}_{m}\left(\check{V}^{2}\right)$ with a similar computation as for $\mathbb{E}_{m}\left(J_{m, 2}\right)$ :

$$
\begin{aligned}
& \mathbb{E}_{m}\left(N_{\mathrm{ex}, 2}\right) \mathbb{E}_{m}\left(\check{V}^{2}\right)=\int_{t_{0}}^{t_{0}+z} \int_{0}^{\infty} \check{V}^{2} \check{r}_{\mathrm{ex}, 2}(\check{V}) \mathrm{d} \check{V} \mathrm{~d} t \\
&= \frac{16 C_{m} S_{d-1}}{(M+m)^{4}} z m^{3 / 2}\left[\int_{\frac{3}{4} m^{1 / 2} c_{m}}^{\infty}\left(\frac{x}{m^{1 / 2}}+\frac{c_{m}}{8}\right)^{3} f(x) \mathrm{d} x\right. \\
&\left.-\int_{m^{1 / 2}\left(\frac{3}{4} c_{m}+\varepsilon\right)}^{\infty}\left(\frac{x}{m^{1 / 2}}+\frac{c_{m}}{8}-\varepsilon\right)^{3} f(x) \mathrm{d} x\right] \\
& \leq \frac{12 S_{d-1}}{M^{2}} z m^{3 / 2} \int_{0}^{\infty} \varepsilon\left(\frac{x}{m^{1 / 2}}+\frac{c_{m}}{8}\right)^{2} f(x) \mathrm{d} x+o\left(m^{1 / 2}\right) \\
& \leq C z m^{1 / 2}+o\left(m^{1 / 2}\right) .
\end{aligned}
$$

We see that this converges to zero as $m \rightarrow 0$.

Combining the above, for $m$ sufficiently small, we have the following estimate on $J_{m, 2}$,

$$
\mathbb{P}\left(\left\{J_{m, 2} \geq \varepsilon / 12\right\} \cap G_{m}\right) \leq \frac{\mathbb{E}_{m}\left(N_{\mathrm{ex}, 2}\right) \mathbb{E}_{m}\left(\check{V}^{2}\right)}{\mathbb{E}_{m}\left(J_{m, 2}\right)^{2}},
$$

and so in view of (D.14) and (D.16),

$$
\lim _{m \rightarrow 0} \mathbb{P}\left(\left\{J_{m, 2} \geq \varepsilon / 12\right\} \cap G_{m}\right)=0 .
$$

Combining the estimates of this section, we conclude

$$
\lim _{m \rightarrow 0} \mathbb{P}\left(\left\{W_{\mathrm{ex}}\left(\tau_{m}^{*}\right) \geq \varepsilon / 6\right\} \cap G_{m}\right) \leq \lim _{m \rightarrow 0} \mathbb{P}\left(\left\{J_{m, 1} \geq \varepsilon / 12\right\} \cap G_{m}\right)+\lim _{m \rightarrow 0} \mathbb{P}\left(\left\{J_{m, 2} \geq \varepsilon / 12\right\} \cap G_{m}\right)=0,
$$

which completes the proof of Lemma D.3.

\section{D.4. Slow collisions}

In this section, we bound the effect of jumps of the mechanical process caused by slow collisions. To do so, we track the total change in the relative velocity of a bath atom, $\overline{\mathbf{v}}=\mathbf{v}-$ Aq. A bath atom that undergoes a slow collision may collide with the large particle many times; however, if the normal component of the bath atom's velocity ever exceeds $c_{m}$ after a collision, then no further collisions are possible, which follows from arguments used in Appendix C.

Suppose a particular bath atom undergoes $k$ collisions with the large particle. We denote by $\overline{\mathbf{v}}_{i}$ (respectively $\overline{\mathbf{v}}_{i}^{\prime}$ ) for $i=1, \ldots, k$ the relative velocity of the bath atom before (respectively after) the $i$ th collision. We show below that the total change in velocity for a bath atom is bounded by $\left|\overline{\mathbf{v}}_{k}^{\prime}-\overline{\mathbf{v}}_{1}\right| \leq 8 c_{m}$. By the preservation of linear momentum for an elastic collision, the change in velocity for the large particle due to all the collisions with this particular bath atom is $\mathbf{V}^{\prime}-\mathbf{V}=\frac{m}{M}\left(\overline{\mathbf{v}}_{k}^{\prime}-\overline{\mathbf{v}}_{1}\right)$. We also show below that the number of unique bath atoms experiencing a slow collision with the large particle in a given finite time interval is bounded above by $C m^{-1 / 2} c_{m}$. Thus, the total expected change in the large particle's velocity is bounded by $C m^{1 / 2} c_{m}^{2}$ which converges to zero as $m \rightarrow 0$. 
Lemma D.5. For $\tau_{m}^{*}$ be given by (D.6), $G_{m}$ given by (D.9), and $W_{\text {slow }}$ defined above in (D.3), we have that

$$
\lim _{m \rightarrow 0} \mathbb{P}\left(\left\{W_{\text {slow }}\left(\tau_{m}^{*}\right) \geq \varepsilon / 6\right\} \cap G_{m}\right)=0 .
$$

Proof. We focus now on a single bath atom and consider the set of times $I_{\text {rec }}=\left\{t_{i}\right\}_{i=1, \ldots, k}$ of all collisions of this single atom in the interval $\left[t_{0}, \tau_{m}^{*}\right]$ (the fact that there are almost surely only finitely many collisions is shown in Appendix E). We later estimate the total number of distinct bath atoms that undergo slow collisions with the large particle in the time interval. We denote by $\mathbf{e}_{\mathrm{n}, i}$ the normal direction for the collision at time $t_{i}$. We denote by $\overline{\mathbf{v}}_{\mathrm{n}, i}, \overline{\mathbf{v}}_{\mathrm{n}, i}^{\prime}$ the normal velocity for the particle before and after the collision at time $t_{i}$, and similarly for the tangential velocity. Finally, we let $\bar{v}_{\mathrm{n}, i}=\overline{\mathbf{v}}_{\mathrm{n}, i} \cdot \mathbf{e}_{\mathrm{n}, i}$.

We recall that in a slow collision, the normal velocity of the bath atom satisfies $\bar{v}_{n} \leq c_{m}$, whereas the tangential velocity cannot be bounded, so we do not have a bound on $|\mathbf{v}|$. In the following, we consider three cases, based on the size of the tangential velocity of the bath atom.

For the first case, we consider that $\left|\overline{\mathbf{v}}_{\mathrm{t}, i}\right|<c_{m}$ for all $i=1, \ldots, k$. The sum of the change in $\overline{\mathbf{v}}$ telescopes since $\overline{\mathbf{v}}_{\mathrm{n}, i}^{\prime}-\overline{\mathbf{v}}_{\mathrm{n}, i}=\overline{\mathbf{v}}_{i}^{\prime}-\overline{\mathbf{v}}_{i}=\overline{\mathbf{v}}_{i+1}-\overline{\mathbf{v}}_{i}$, giving

$$
\left|\sum_{i=1}^{k} \overline{\mathbf{v}}_{\mathrm{n}, i}^{\prime}-\overline{\mathbf{v}}_{\mathrm{n}, i}\right|=\left|\sum_{i=1}^{k} \overline{\mathbf{v}}_{i}^{\prime}-\overline{\mathbf{v}}_{i}\right|=\left|\overline{\mathbf{v}}_{k}^{\prime}-\overline{\mathbf{v}}_{1}\right| \leq 2 \sqrt{2} c_{m} .
$$

As a second case, suppose that $\left|\overline{\mathbf{v}}_{\mathrm{t}, 1}\right| \geq c_{m}$. Even with a large tangential velocity, it is possible that there is a recollision with the large particle due to the curvature of the particle (see Fig. 8). We split the large particle into halves, with the equator perpendicular to $\mathbf{e}_{\mathrm{t}_{1}}$ as in Figure 8. The bath atom passes the equator and due to its high tangential velocity, which is unchanged by the collision, all subsequent collisions must occur on the same hemisphere (the shaded hemisphere in Fig. 8a). We recall that the speed of the large particle is

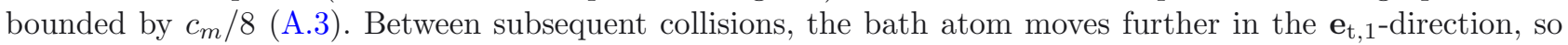
that $\left\langle\mathbf{e}_{\mathrm{t}, 1}, \mathbf{e}_{\mathrm{n}, i+1}\right\rangle \leq\left\langle\mathbf{e}_{\mathrm{t}, 1}, \mathbf{e}_{\mathrm{n}, i}\right\rangle$ and therefore $\left\langle\mathbf{e}_{\mathrm{t}, 1}, \mathbf{e}_{\mathrm{n}, i}\right\rangle \leq 0$ for all $i$. This in turn implies monotonicity of the normal velocity for the bath atom's collisions,

$$
\bar{v}_{\mathrm{n}, i+1} \leq \bar{v}_{\mathrm{n}, i}^{\prime}
$$

From Lemma C.1, any bath atom involved in a fast collision can never undergo slow collisions, so in particular we have that $\left|\overline{\mathbf{v}}_{\mathrm{n}, i}\right| \leq c_{m}$ for $i=1, \ldots, k$. We use the monotonicity of the normal velocities (D.17) to form a telescopic sum on the normal component of the change in velocity, which becomes

$$
\begin{aligned}
\left|\sum_{i=1}^{k} \overline{\mathbf{v}}_{\mathrm{n}, i}^{\prime}-\overline{\mathbf{v}}_{\mathrm{n}, i}\right| & \leq \sum_{i=1}^{k-1}\left|\overline{\mathbf{v}}_{\mathrm{n}, i+1}-\overline{\mathbf{v}}_{\mathrm{n}, i}\right|+\left|\overline{\mathbf{v}}_{\mathrm{n}, k}^{\prime}-\overline{\mathbf{v}}_{\mathrm{n}, k}\right| \\
& \leq \bar{v}_{\mathrm{n}, 1}-\bar{v}_{\mathrm{n}, \mathrm{k}}+\left|\overline{\mathbf{v}}_{\mathrm{n}, k}^{\prime}-\overline{\mathbf{v}}_{\mathrm{n}, k}\right| \\
& \leq 2 c_{m}+2\left(c_{m}+c_{m} / 16\right) \leq 5 c_{m} .
\end{aligned}
$$

To prove the third inequality, we use the bound on $\left|\bar{v}_{\mathrm{n}, i}\right| \leq c_{m}$ for $i=0, \ldots, k$, along with the bound on the large particle in (A.3), and apply the collision rule (2.7).

For the third and final case, consider the case that after a certain number of collisions, $\left|\overline{\mathbf{v}}_{\mathrm{t}, \ell}\right| \geq c_{m}$ whereas until that time, we have that $\left|\overline{\mathbf{v}}_{\mathrm{t}, i}\right|<c_{m}$ and $\left|\overline{\mathbf{v}}_{\mathrm{n}, i}\right|<c_{m}$, for $i=1, \ldots, \ell-1$. This combines the previous two cases. The change in velocity satisfies

$$
\begin{aligned}
\left|\sum_{i=1}^{k} \overline{\mathbf{v}}_{\mathrm{n}, i}^{\prime}-\overline{\mathbf{v}}_{\mathrm{n}, i}\right| & =\left|\sum_{i=1}^{\ell-1} \overline{\mathbf{v}}_{\mathrm{n}, i}^{\prime}-\overline{\mathbf{v}}_{\mathrm{n}, i}\right|+\left|\sum_{i=\ell}^{k} \overline{\mathbf{v}}_{\mathrm{n}, i}^{\prime}-\overline{\mathbf{v}}_{\mathrm{n}, i}\right| \\
& =\left|\overline{\mathbf{v}}_{\ell-1}^{\prime}-\overline{\mathbf{v}}_{1}\right|+\left|\sum_{i=\ell}^{k} \overline{\mathbf{v}}_{\mathrm{n}, i}^{\prime}-\overline{\mathbf{v}}_{\mathrm{n}, i}\right| \\
& \leq 2 \sqrt{2} c_{m}+5 c_{m} \leq 8 c_{m} .
\end{aligned}
$$




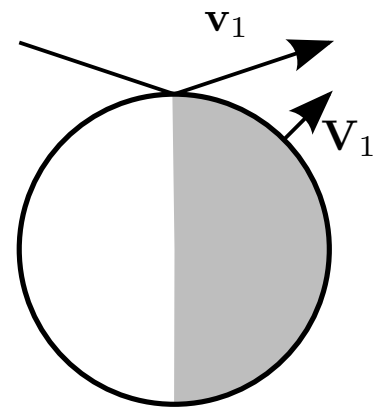

(a)

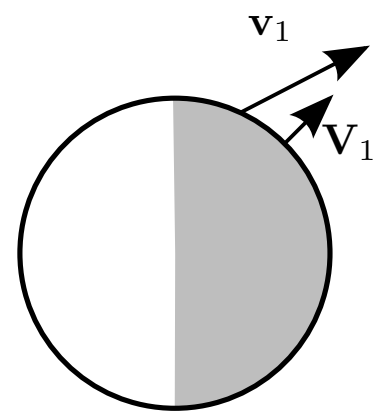

(b)

Figure 8. A slow collision with high tangential velocity in the plane defined by the bath atom's tangential and normal velocities. (a) If the tangential velocity of the first collision is large all subsequent collisions must occur in one hemisphere, in the positive $\mathbf{e}_{t, 1}$-direction. (b) The later collisions have normal velocity smaller and smaller, since the large component in the $\mathbf{e}_{t, 1}$ direction is outward pointing from the surface of the particle at the site of subsequent collisions.

We estimate $\left|\overline{\mathbf{v}}_{1}\right| \leq \sqrt{2} c_{m}$ and $\left|\overline{\mathbf{v}}_{\ell-1}^{\prime}\right| \leq \sqrt{2} c_{m}$ as both normal and tangential components of the relative velocity are bounded. We bound the final term, involving collisions after the fast tangential velocity using the previous argument (D.18).

Taking the maximum over the above three estimates, we conclude that the total momentum change for the large particle due to collisions with this single, slow particle satisfies

$$
\left|\sum_{i=1}^{k} M\left(\mathbf{V}_{\mathrm{n}, i}^{\prime}-\mathbf{V}_{\mathrm{n}, i}\right)\right|=\left|\sum_{i=1}^{k} m\left(\overline{\mathbf{v}}_{\mathrm{n}, i}^{\prime}-\overline{\mathbf{v}}_{\mathrm{n}, i}\right)\right| \leq 8 m c_{m}
$$

We now define $N_{\text {slow }}$ as the number of first-time collisions that are slow collisions, and we employ a doubleindex notation for the collisions, so that the $j$ th atom's $i$ th collision has relative normal velocity $\overline{\mathbf{v}}_{\mathrm{n}, i, j}$ where $1 \leq j \leq N_{\text {slow }}$ and $1 \leq i \leq k_{j}$. While the slow collisions are not a Poisson jump process, we note (A.4) acts as an upper bound for the first-time slow collisions. (We recall that in the mechanical process certain slow collisions are made impossible by the past history, though no first-time slow collisions are ignored in (A.4)). Then we may estimate the number of distinct bath atoms involved in slow collisions by

$$
\begin{aligned}
\mathbb{E}_{m}\left(N_{\text {slow }}\right) & \leq z \int_{\mathbb{S}^{d-1}} \int_{0}^{c_{m}} \frac{\lambda}{m^{1 / 2}} R^{d-1}\left(v_{\mathrm{n}}-V_{\mathrm{n}}\right)_{+} f_{m}^{1}\left((\mathbf{v}-A \mathbf{q})_{\mathrm{n}}\right) \mathrm{d} v_{\mathrm{n}} \mathrm{d} \Omega \\
& \leq \lambda R^{d-1} S_{d-1} m^{-1 / 2} z \int_{0}^{c_{m}} 2 c_{m} f_{m}^{1}\left((\mathbf{v}-A \mathbf{q})_{\mathrm{n}}\right) \mathrm{d} v_{\mathrm{n}} \\
& \leq 2 \lambda R^{d-1} S_{d-1} m^{-1 / 2} z c_{m} .
\end{aligned}
$$


Combining (D.19) and (D.20), we finally have the estimate

$$
\begin{aligned}
\mathbb{P}\left(\left\{W_{\text {slow }}\left(\tau_{m}^{*}\right) \geq \varepsilon / 6\right\} \cap G_{m}\right) & \leq \mathbb{P}\left(\frac{m}{M}\left|\sum_{j=1}^{N_{\text {slow }}} \sum_{i=1}^{k_{i}} \overline{\mathbf{v}}_{\mathrm{n}, i, j}^{\prime}-\overline{\mathbf{v}}_{\mathrm{n}, i, j}\right| \geq \varepsilon / 6\right) \\
& \leq \mathbb{P}\left(\frac{8 m c_{m}}{M} N_{\text {slow }} \geq \varepsilon / 6\right) \\
& \leq \frac{48 m c_{m}}{\varepsilon} \mathbb{E}_{m}\left(N_{\text {slow }}\right) \\
& \leq \frac{96 \lambda R^{d-1} S_{d-1} z c_{m}^{2} m^{1 / 2}}{\varepsilon} \rightarrow 0 \text { as } m \rightarrow 0 .
\end{aligned}
$$

\section{D.5. Conclusion of the proof}

Combining the above estimates on $W_{\text {slow }}$ and $W_{\text {ex }}$, we conclude that the left hand side of (D.12) converges to zero in the limit as $m \rightarrow 0$. This shows that Lemma D.2 holds, and we then conclude that Lemma D.1 follows. Over finite time intervals, we can then conclude that $\left(\widetilde{\mathbf{Q}}_{m}, \widetilde{\mathbf{V}}_{m}\right)_{\{0 \leq t \leq T\}}-\left(\mathbf{Q}_{m}, \mathbf{V}_{m}\right)_{\{0 \leq t \leq T\}} \stackrel{p}{\longrightarrow} 0$ as $m \rightarrow 0$. Combined with the convergence result Lemma B.1, we can therefore conclude the Proof of our main result, Theorem 2.4.

\section{Appendix E. The Mechanical process is Well-Posed}

In this section, we show that the mechanical process is almost surely well-posed for all times $t<\tau_{m}$, where $\tau_{m}$ is the stopping time defined in (A.3). Particularly, we show that as long as the large particle stays bounded, the degenerate cases of having a multicollision or having infinitely many collisions in finite time both occur with zero probability. We further show that almost surely $\tau_{m}>0$. We note that this well-posedness result is slightly weaker than the corresponding result in $[6,7]$ for a heat bath with zero background flow since that result does not require a stopping time and is valid over finite time intervals that are uniform with respect to the initial condition. In contrast, the stopping time $\tau_{m}$ depends on the initial condition.

We first show that whenever the particle is restricted to a ball $\left|\mathbf{Q}_{m}\right|<\ell$ for $\ell \in \mathbb{N}$, then there are almost surely only finitely many atoms that can possibly interact with the particle in a given time interval $[0, T]$, since only finitely many enter the ball. It is therefore sufficient to study the well-posedness of the dynamics for finite systems. After restricting to a finite system, we show that the mechanical process is almost surely well defined. Since $\left|\mathbf{Q}_{m}\right|<c_{m} / 8$ for $t \in\left[0, \tau_{m}\right)$, we conclude that the mechanical process is well-posed at least until the stopping time $\tau_{m}$.

\section{E.1. Finitely many atoms within a finite radius}

In the following, we work with fixed mass $m$ for the bath atoms, which we absorb into other constants.

Lemma E.1. For any finite time interval $[0, T]$ and radius $\ell \in \mathbb{N}$, there are almost surely only finitely many bath atoms that enter the ball $|\mathbf{q}|<\ell$ in the time interval $[0, T]$. 
Proof. For a given initial configuration, let $N_{k}$ be the number of bath atoms such that $k<|\mathbf{q}| \leq k+1$ and $|\overline{\mathbf{v}}| \geq \varepsilon k$, where we choose $\varepsilon=\frac{1}{2} \mathrm{e}^{-\left(\|A\|+\frac{1}{2}\right) T}$. We then have

$$
\begin{aligned}
\mathbb{E}_{m}\left(N_{k}\right) & =\int_{k \leq|\mathbf{q}| \leq k+1} \int_{|\overline{\mathbf{v}}| \geq \varepsilon k} \lambda_{m} f_{m}(\mathbf{q}, \overline{\mathbf{v}}+A \mathbf{q}) \mathrm{d} \overline{\mathbf{v}} \mathrm{d} \mathbf{q} \\
& =C\left[(k+1)^{d}-k^{d}\right] S_{d-1} \int_{|\overline{\mathbf{v}}| \geq \varepsilon k} \frac{|\overline{\mathbf{v}}|^{d+1}}{|\overline{\mathbf{v}}|^{d+1}} f\left(m^{1 / 2} \overline{\mathbf{v}}\right) \mathrm{d} \overline{\mathbf{v}} \\
& \leq \frac{C k^{d-1}}{(\varepsilon k)^{d+1}} \int_{|\overline{\mathbf{v}}| \geq m^{1 / 2} \varepsilon k}|\overline{\mathbf{v}}|^{d+1} f(\overline{\mathbf{v}}) \mathrm{d} \overline{\mathbf{v}} \\
& \leq \frac{C}{k^{2}}
\end{aligned}
$$

where we note that $C$ depends on $\varepsilon$, but not on $k$. Then, almost surely $\sum_{k=1}^{\infty} N_{k}<\infty$. Suppose an atom has initial coordinates $\left(\mathbf{q}_{0}, \overline{\mathbf{v}}_{0}\right)$ where $k \leq\left|\mathbf{q}_{0}\right| \leq k+1$ and $\left|\overline{\mathbf{v}}_{0}\right|<\varepsilon k$ for some $k \in \mathbb{N}$. We have from the dynamics $(2.20)$ that

$$
\begin{aligned}
\frac{\mathrm{d}}{\mathrm{d} t}\left(\frac{|\mathbf{q}|^{2}}{2}\right) & =\mathbf{q}^{T} A \mathbf{q}+\mathbf{q}^{T} \overline{\mathbf{v}}_{0} \\
& \geq-\|A\||\mathbf{q}|^{2}-\frac{1}{2}|\mathbf{q}|^{2}-\frac{1}{2}\left|\overline{\mathbf{v}}_{0}\right|^{2}
\end{aligned}
$$

This implies

$$
\begin{aligned}
|\mathbf{q}(t)|^{2} & \geq\left(\left|\mathbf{q}_{0}\right|^{2}+\frac{\left|\overline{\mathbf{v}}_{0}\right|^{2}}{2\|A\|+1}\right) \mathrm{e}^{-(2\|A\|+1) t}-\frac{\left|\overline{\mathbf{v}}_{0}\right|^{2}}{2\|A\|+1} . \\
& \geq\left|\mathbf{q}_{0}\right|^{2} \mathrm{e}^{-(2\|A\|+1) t}-\left|\overline{\mathbf{v}}_{0}\right|^{2} \\
& \geq k^{2} \mathrm{e}^{-(2\|A\|+1) t}-\varepsilon^{2} k^{2} \\
& =\frac{3}{4} k^{2} \mathrm{e}^{-(2\|A\|+1) T} .
\end{aligned}
$$

Let $\ell \in \mathbb{N}$ be fixed. We choose $k_{0}$ sufficiently large such that $\frac{\sqrt{3}}{2} k_{0} \mathrm{e}^{-\left(\|A\|+\frac{1}{2}\right) T}>\ell$. Then, the only atoms that can enter the ball $|\mathbf{q}|<\ell$ must either be one of the finitely many such that $\left|\mathbf{q}_{0}\right|<k_{0}$ or be such that there exists $k \geq k_{0}$ such that $k<\left|\mathbf{q}_{0}\right| \leq k+1$ and $\left|\overline{\mathbf{v}}_{0}\right| \geq \varepsilon k$.

In a finite mechanical system, the first collision occurs at a time $t_{0}>0$, since at time $t=0$, no bath atoms are touching the large particle. Upon choosing $\ell=\frac{c_{m}}{8}$, this implies that the stopping time $\tau_{m}$ (A.3) for the mechanical process satisfies $\tau_{m}>0$. (Recall that after equation (A.3), we have assumed that $m$ is sufficiently small so that the initial conditions lie within the box that defines the stopping criteria.)

\section{E.2. A finite system is well-posed}

In the following, we consider a system composed of the large particle along with $N \in \mathbb{N}$ bath atoms, in the finite time interval $\left[0, \tau_{m}\right)$. We show that there are almost surely only finitely many collisions, none of which are multicollisions. For any initial condition of the mechanical process we define the process on the time interval $\left[0, \tau^{*}\right)$ where $\tau^{*}$ is the first occurrence of either a multicollision or an accumulation of infinitely many collisions, or the first time the large particle leaves the box used in the definition of $\tau_{m}$ (see (A.3)). By definition, the mechanical process is well-posed on the time interval $\left[0, \tau^{*}\right)$, and we have $\tau^{*} \leq \tau_{m}$. From Lemma E.1, we can conclude that $\tau^{*}>0$, and we show below that almost surely $\tau^{*}=\tau_{m}$.

We note that because of the bath atom dynamics (2.18), the kinetic energy of the mechanical process is not preserved. When restricting to a finite system, we can control the growth of the energy. 
Lemma E.2. In the time interval $\left[0, \tau^{*}\right)$, the kinetic energy of the mechanical system at time $t$,

$$
K(t)=\frac{1}{2} M \mathbf{V}(t)^{T} \mathbf{V}(t)+\frac{1}{2} \sum_{j=1}^{N} m \mathbf{v}_{j}(t)^{T} \mathbf{v}_{j}(t),
$$

satisfies $K(t) \leq K(0) \mathrm{e}^{2\|A\| t}$.

Proof. During an elastic collision, the kinetic energy is unchanged. Between collisions, we have

$$
\begin{aligned}
\frac{\mathrm{d}}{\mathrm{d} t} K(t) & =M \mathbf{V}(t)^{T} \frac{\mathrm{d}}{\mathrm{d} t} \mathbf{V}(t)+\sum_{j=1}^{N} m \mathbf{v}_{j}(t)^{T} \frac{\mathrm{d}}{\mathrm{d} t} \mathbf{v}_{j}(t) \\
& =\sum_{j=1}^{N} m \mathbf{v}_{j}(t)^{T} A \mathbf{v}_{j}(t)
\end{aligned}
$$

and we thus have $\left|\frac{\mathrm{d}}{\mathrm{d} t} K(t)\right| \leq 2\|A\| K(t)$. Integrating gives the desired result.

As a corollary, we have that for every initial condition, the speed $v_{\max }=\left(\frac{K(0) \mathrm{e}^{2\|A\| \tau^{*}}}{m}\right)^{1 / 2}$ bounds the maximum speed of any bath atom:

$$
\max _{t \in\left[0, \tau^{*}\right)} \max _{j=1, \ldots, N}\left|\mathbf{v}_{i}(t)\right| \leq v_{\max }
$$

We let $B_{N}(\ell, K)$ denote the set of initial conditions where $\tau^{*}<\tau_{m}$, that is, those that lead to either a multicollision or infinitely many collisions, where $N$ is the number of bath atoms in the system, where the large particle is restricted to $|\mathbf{Q}| \leq \ell$, and where the total kinetic energy satisfies $\sup _{[0, T]} K(t) \leq K$. If we show that the set $B_{N}(\ell, K)$ has zero measure, then the set of initial conditions leading to such 'bad' collisions, which is given by

$$
\bigcup_{N=1}^{\infty} \bigcup_{\ell=1}^{\infty} \bigcup_{K=1}^{\infty} B_{N}(\ell, K)
$$

has zero measure.

We first restrict ourselves to the case of a system with the large particle and a single bath atom.

Lemma E.3. For all initial conditions in $B_{1}(\ell, K)$, there are infinitely many collisions in $\left[0, \tau^{*}\right)$ and the normal component of the atom and particle velocities before the ith collision satisfies

$$
\lim _{i \rightarrow \infty}\left|v_{\mathrm{n}, i}-V_{\mathrm{n}, i}\right|=0 .
$$

Proof. Since there is only one bath atom, the only possible bad collision is the accumulation of infinitely many collisions. Suppose a collision occurs with relative velocity $v_{\mathrm{n}, i}-V_{\mathrm{n}, i}=\varepsilon$, for some $\varepsilon>0$. Immediately after the collision $v_{\mathrm{n}, i}^{\prime}-V_{\mathrm{n}, i}^{\prime}=-\varepsilon$. Since the system is composed of only a single particle and a single bath atom, in order for another collision to occur, the bath atom must accelerate until $(\mathbf{v}(t)-\mathbf{V}(t)) \cdot \mathbf{e}_{\mathrm{n}, i} \geq 0$.

From the bound on the bath atom velocity in (E.1),

$$
\frac{\mathrm{d}}{\mathrm{d} t} \mathbf{v}(t)=A \mathbf{v} \leq\|A\| v_{\max } .
$$

We conclude that there exists $\delta>0$ such that the time between collisions satisfies $t_{i+1}-t_{i} \geq \delta \varepsilon$. Thus, an accumulation of collisions implies that $\lim _{i \rightarrow \infty}\left|v_{\mathrm{n}, i}-V_{\mathrm{n}, i}\right|=0$. 
Lemma E.4. The set $B_{1}(\ell, K)$ has measure zero.

Proof. For any $\varepsilon>0$, let $\tau_{\varepsilon}^{*}$ denote the time of the first collision where $\left|v_{\mathrm{n}, i}-V_{\mathrm{n}, i}\right|<\varepsilon$. For $P \in \mathbb{N}$, let $\Gamma_{\varepsilon, p}^{P} \subset B_{1}(\ell, K)$ be the set of initial conditions where $\tau_{\varepsilon}^{*} \in\left[\frac{p}{P} \tau_{m}, \frac{p+1}{P} \tau_{m}\right)$, for $p=0, \ldots, P-1$. By Lemma E.3, an accumulation of collisions must lead to slow collisions, so that $B_{1}(\ell, K) \subset \cup_{p=0}^{P-1} \Gamma_{\varepsilon, p}^{P}$.

Up to time $\tau_{\varepsilon}^{*}$, the dynamics is well-defined, and the flow map $\phi_{t}$ for the mechanical process preserves Lebesgue measure. For $p=1, \ldots, P-1, \phi_{\frac{p}{P} \tau_{m}}\left(\Gamma_{\varepsilon, p}^{P}\right) \subset \Gamma_{\varepsilon, 0}^{P}$, so that $\left|\Gamma_{\varepsilon, p}^{P}\right| \leq\left|\Gamma_{\varepsilon, 0}^{P}\right|$.

For initial conditions in $\Gamma_{\varepsilon, 0}^{P}$, we may suppose that $P^{-1}$ is sufficiently small so that $\tau_{\varepsilon}^{*}$ is the time of the first collision (recall from Lemma E.3 that a collision with $\left|v_{\mathrm{n}, i}-V_{\mathrm{n}, i}\right| \geq \varepsilon$ leads to a minimum time between collisions $t_{i+1}-t_{i} \geq \delta \varepsilon$ ). Mapping back from a collision at time $\tau_{\varepsilon}^{*}$, we see that the bath atom's initial position satisfies $|\mathbf{q}-\mathbf{Q}| \leq \frac{v_{\max } \tau_{m}}{P}$ and the initial velocity satisfies $\left|v_{\mathrm{n}, i}-V_{\mathrm{n}, i}\right| \leq \varepsilon \mathrm{e}^{\|A\| \frac{\tau_{m}}{P}} v_{\max }$. Because of the bounds on $\mathbf{Q}$ and $K$, we then conclude that $\left|\Gamma_{\varepsilon, 0}^{P}\right| \leq \frac{C_{1} \varepsilon}{P} \mathrm{e}^{\frac{C_{2}}{P}}$, where $C_{1}, C_{2} \in \mathbb{R}$ are independent of $\varepsilon$ and $P$. Summing over $p=0, \ldots, P-1$, we have

$$
\left|B_{1}(\ell, K)\right| \leq \lim _{\varepsilon \rightarrow 0} \lim _{P \rightarrow \infty}\left|\bigcup_{p=0}^{P-1} \Gamma_{\varepsilon, p}^{P}\right|=0 .
$$

Lemma E.5. The subset $M \subset B_{N}(\ell, K)$ of initial conditions where the first bad collision is a multicollision has measure zero.

Proof. Intuitively, the set of all multicollisions at a given instant $\tau^{*}$ has phase space co-dimension greater than or equal to two, so that the set of initial conditions leading to a multicollision has zero measure. We note that a multicollision could involve one or more bath atoms that have an accumulation of infinitely many collisions at time $\tau^{*}$.

For $P \in \mathbb{N}$, we let $\Gamma_{\varepsilon, p}^{P}$ be the set of initial conditions such that $\tau^{*} \in\left[\frac{p}{P} \tau_{m}, \frac{p+1}{P} \tau_{m}\right)$, where $p=0, \ldots, P-1$, where we recall that $\tau^{*}$ is the time of the first multicollision. We note that since the flow map of the mechanical process preserves Lebesgue measure, $\left|\Gamma_{\varepsilon, p}^{P}\right| \leq\left|\Gamma_{\varepsilon, 0}^{P}\right|$, so we only need to bound the measure of $\Gamma_{\varepsilon, 0}^{P}$.

For initial conditions in $\Gamma_{\varepsilon, 0}^{P}$, two or more bath atoms must satisfy $|\mathbf{q}-\mathbf{Q}| \leq \frac{v_{\max } \tau_{m}}{P}$, so that we have $\left|\Gamma_{\varepsilon, 0}^{P}\right| \leq \frac{C}{P^{2}}$. We thus have

$$
|M|=\lim _{P \rightarrow \infty}\left|\bigcup_{p=0}^{P-1} \Gamma_{\varepsilon, p}^{P}\right|=0 .
$$

Lemma E.6. The set of initial conditions leading to a bad collision has measure zero.

Proof. We first consider the full set $B_{N}(\ell, K)$. If the first bad collision in such a system is not a multicollision, then there is a time $\delta$ such that in the interval $\left[\tau^{*}-\delta, \tau^{*}\right)$, the only collisions that the large particle experiences are with the bath atom that experiences the bad collision at $\tau^{*}$. In this case, the arguments from Lemma E.4 show that the set of such initial conditions has measure zero. Therefore, using Lemma E.5, we conclude that $B_{N}(\ell, K)$ has measure zero as well.

We then conclude that

$$
\left|\bigcup_{N=1}^{\infty} \bigcup_{\ell=1}^{\infty} \bigcup_{K=1}^{\infty} B_{N}(\ell, K)\right|=0,
$$

and that almost surely $\tau^{*}=\tau_{m}$ and so the mechanical process is well-posed until at least the stopping time $\tau_{m}$.

\section{Appendix F. Convergence of the Generator for the laminar heat Bath}

In this section, we compute the limiting SDE for the two laminar heat bath models defined in Section 2.3. The computations parallel those of Appendix B, and we omit some of the details found there. The first heat 
bath model has velocity distribution of the form (2.11), and the second has distribution of the form (2.14) where we consider the specific shear flow

$$
A=\left[\begin{array}{lll}
0 & s & 0 \\
0 & 0 & 0 \\
0 & 0 & 0
\end{array}\right]
$$

for some $s \in \mathbb{R}$. We do not carry out a full convergence proof as we do for the model proposed in Section 2.4 - although such a proof could likely be carried out based on the same arguments as above - since the limiting dynamics obtained by the following formal computations has undesirable traits for sampling nonequilibrium states.

\section{F.1. Unidirectional flow}

We start with a background bath whose atoms are distributed initially according to (2.11), where all bath atom velocities at initial time are oriented in the $\mathbf{e}_{1}$-direction. In fact, rather than working with (2.11) and its Gaussian distribution, we work with

$$
\mathrm{d} \mu_{m}\left(\mathbf{q}, v_{1}\right)=\lambda_{m} f_{m}\left(v_{1}-s q_{2}\right) \mathrm{d} \mathbf{q} \mathrm{d} v_{1},
$$

where we have the scaling $f_{m}(\bar{v})=m^{1 / 2} f\left(m^{1 / 2} \bar{v}\right)$, where the probability density function $f(\bar{v})$ is assumed to be decreasing in $\bar{v}$ with four finite moments (2.3), and where we recall $q_{2}=Q_{2}-R \mathbf{e}_{n} \cdot \mathbf{e}_{2}$. Note that we have eliminated $v_{2}$ and $v_{3}$ in the above expression since they are identically zero. We recover (2.11) upon choosing $f(x)=Z^{-1} \exp \left(-\frac{\beta}{2} x^{2}\right)$.

The normal velocity for a bath atom before a collision is $v_{n}=v_{1} \mathbf{e}_{1} \cdot \mathbf{e}_{\mathrm{n}}$, and we keep the distinction between slow and fast collisions of Definition A.1. The rate of fast collisions experienced by the particle is

$$
\begin{aligned}
r_{m}\left(v_{1}, \mathbf{e}_{\mathrm{n}}, \mathbf{Q}, \mathbf{V}\right) \mathrm{d} v_{1} \mathrm{~d} \Omega \mathrm{d} t= & \lambda_{m} R^{2} H\left(\left(v_{1} \mathbf{e}_{1}-s q_{2}\right) \cdot \mathbf{e}_{\mathrm{n}}-c_{m}\right) \\
& \times \max \left(v_{\mathrm{n}}-V_{\mathrm{n}}, 0\right) f_{m}\left(v_{1}-s q_{2}\right) \mathrm{d} v_{1} \mathrm{~d} \Omega \mathrm{d} t .
\end{aligned}
$$

In contrast to (A.4), the rate function here includes a Heaviside function to ignore slow collisions. We let $\widehat{V}=V_{n}^{\prime}-V_{n}$, which satisfies, in view of $(2.7)$

$$
\begin{aligned}
\widehat{V} & =\frac{2 m}{M+m}\left(v_{1} \mathbf{e}_{1} \cdot \mathbf{e}_{\mathrm{n}}-V_{\mathrm{n}}\right), \\
\widehat{V}_{\min } & =\frac{2 m}{M+m}\left(c_{m}+s q_{2} \mathbf{e}_{1} \cdot \mathbf{e}_{\mathrm{n}}-V_{\mathrm{n}}\right),
\end{aligned}
$$

where $\widehat{V}_{\text {min }}$ denotes the minimum velocity jump size (due to the cutoff for slow collisions). For $\widehat{V} \geq \widehat{V}_{\text {min }}$, the measure on jumps $\widehat{V} \mathbf{e}_{\mathrm{n}}$ is

$$
\hat{r}_{m}\left(\widehat{V}, \mathbf{e}_{\mathrm{n}}, \mathbf{Q}, \mathbf{V}\right)=\lambda_{m} R^{2}\left(\frac{M+m}{2 m}\right)^{2} \frac{\widehat{V}}{\left|\mathbf{e}_{1} \cdot \mathbf{e}_{\mathrm{n}}\right|} f_{m}\left(\frac{1}{\mathbf{e}_{1} \cdot \mathbf{e}_{\mathrm{n}}}\left[\frac{M+m}{2 m} \widehat{V}+V_{\mathrm{n}}\right]-s q_{2}\right) .
$$

Using this jump measure, we define a Markov process as in Definition A.5.

We write the generator for the Markov process

$$
L_{m} \psi=\mathbf{V} \cdot \nabla_{Q} \psi+\int_{\mathbb{S}^{2}} \int_{\widehat{V}_{\min }}^{\infty}\left[\psi\left(\mathbf{Q}, \mathbf{V}+\widehat{V} \mathbf{e}_{\mathrm{n}}\right)-\psi(\mathbf{Q}, \mathbf{V})\right] \hat{r}_{m}\left(\widehat{V}, \mathbf{e}_{\mathrm{n}}, \mathbf{Q}, \mathbf{V}\right) \mathrm{d} \widehat{V} \mathrm{~d} \Omega .
$$

From the scaling of bath atom velocities and associated law (F.1), $\mathbb{E}_{m}(|\mathbf{v}|)$ is $O\left(m^{-1 / 2}\right)$ and by (F.2) we have that $\mathbb{E}_{m}(\widehat{V})$ is $O\left(m^{1 / 2}\right)$. We expand the generator for the Markov process in powers of $\widehat{V}$ and find to leading order

$$
L_{m} \psi=\mathbf{V} \cdot \nabla_{Q} \psi(\mathbf{Q}, \mathbf{V})+C_{m}\left(I_{1} \cdot \nabla_{V} \psi(\mathbf{Q}, \mathbf{V})+\frac{1}{2} I_{2}: \nabla_{V}^{2} \psi(\mathbf{Q}, \mathbf{V})\right)+O\left(m^{1 / 2}\right),
$$


where

$$
\begin{aligned}
C_{m} & =\lambda R^{2}\left(\frac{M+m}{2}\right)^{2}, \\
I_{1} & =m^{-5 / 2} \int_{\mathbb{S}^{2}} \int_{\widehat{V}_{\min }}^{\infty} \mathbf{e}_{\mathrm{n}} \frac{\widehat{V}^{2}}{\left|\mathbf{e}_{1} \cdot \mathbf{e}_{\mathrm{n}}\right|} f_{m}\left(\frac{1}{\mathbf{e}_{1} \cdot \mathbf{e}_{\mathrm{n}}}\left[\frac{M+m}{2 m} \widehat{V}+V_{\mathrm{n}}\right]-s q_{2}\right) \mathrm{d} \widehat{V} \mathrm{~d} \Omega, \\
I_{2} & =m^{-5 / 2} \int_{\mathbb{S}^{2}} \int_{\widehat{V}_{\min }}^{\infty}\left(\mathbf{e}_{\mathrm{n}} \otimes \mathbf{e}_{\mathrm{n}}\right) \frac{\widehat{V}^{3}}{\left|\mathbf{e}_{1} \cdot \mathbf{e}_{\mathrm{n}}\right|} f_{m}\left(\frac{1}{\mathbf{e}_{1} \cdot \mathbf{e}_{\mathrm{n}}}\left[\frac{M+m}{2 m} \widehat{V}+V_{\mathrm{n}}\right]-s q_{2}\right) \mathrm{d} \widehat{V} \mathrm{~d} \Omega,
\end{aligned}
$$

where $q_{2}=Q_{2}-R\left(\mathbf{e}_{\mathrm{n}} \cdot \mathbf{e}_{2}\right)$. Note that estimating the remainder term follows as in Appendix B.1.1.

In both integrals $I_{1}$ and $I_{2}$, we substitute

$$
\begin{aligned}
x & =\frac{1}{\left|\mathbf{e}_{1} \cdot \mathbf{e}_{n}\right|}\left[\frac{M+m}{2 m^{1 / 2}} \widehat{V}+m^{1 / 2} V_{\mathrm{n}}\right]-m^{1 / 2} s \frac{\mathbf{e}_{1} \cdot \mathbf{e}_{\mathrm{n}}}{\left|\mathbf{e}_{1} \cdot \mathbf{e}_{\mathrm{n}}\right|} q_{2}, \\
x_{\text {min }} & =\frac{m^{1 / 2} c_{m}}{\left|\mathbf{e}_{1} \cdot \mathbf{e}_{n}\right|},
\end{aligned}
$$

where $x_{\min }>0$ is the minimum value of $x$. The drift term, $I_{1}$, becomes

$$
\begin{aligned}
I_{1}= & m^{-1 / 2} \int_{\mathbb{S}^{2}} \int_{x_{\min }}^{\infty} \mathbf{e}_{\mathrm{n}}\left(\left|\mathbf{e}_{1} \cdot \mathbf{e}_{\mathrm{n}}\right| x-m^{1 / 2} V_{\mathrm{n}}+m^{1 / 2} s\left(\mathbf{e}_{1} \cdot \mathbf{e}_{\mathrm{n}}\right) q_{2}\right)^{2} \frac{8}{(M+m)^{3}} f\left(\frac{\left|\mathbf{e}_{1} \cdot \mathbf{e}_{\mathrm{n}}\right|}{\mathbf{e}_{1} \cdot \mathbf{e}_{\mathrm{n}}} x\right) \mathrm{d} x \mathrm{~d} \Omega \\
= & \frac{8 m^{-1 / 2}}{M^{3}}\left[\int_{\mathbb{S}^{2}} \int_{x_{\min }}^{\infty} \mathbf{e}_{\mathrm{n}}\left(\left|\mathbf{e}_{1} \cdot \mathbf{e}_{\mathrm{n}}\right| x\right)^{2} f\left(\frac{\left|\mathbf{e}_{1} \cdot \mathbf{e}_{\mathrm{n}}\right|}{\mathbf{e}_{1} \cdot \mathbf{e}_{\mathrm{n}}} x\right) \mathrm{d} x \mathrm{~d} \Omega\right. \\
& \left.+2 \int_{\mathbb{S}^{2}} \int_{0}^{\infty} \mathbf{e}_{\mathrm{n}}\left(-m^{1 / 2} V_{\mathrm{n}}+m^{1 / 2} s\left(\mathbf{e}_{1} \cdot \mathbf{e}_{\mathrm{n}}\right) q_{2}\right)\left(\left|\mathbf{e}_{1} \cdot \mathbf{e}_{\mathrm{n}}\right| x\right) f\left(\frac{\left|\mathbf{e}_{1} \cdot \mathbf{e}_{\mathrm{n}}\right|}{\mathbf{e}_{1} \cdot \mathbf{e}_{\mathrm{n}}} x\right) \mathrm{d} x \mathrm{~d} \Omega\right]+O\left(m^{3 / 10}\right) \\
= & \frac{16 \Phi_{1}}{M^{3}}\left[\int_{\mathbb{S}^{2}} \mathbf{e}_{\mathrm{n}}\left(-\mathbf{V} \cdot \mathbf{e}_{\mathrm{n}}+s\left(\mathbf{e}_{1} \cdot \mathbf{e}_{\mathrm{n}}\right) q_{2}\right)\left|\mathbf{e}_{1} \cdot \mathbf{e}_{\mathrm{n}}\right| \mathrm{d} \Omega\right]+O\left(m^{3 / 10}\right),
\end{aligned}
$$

where we have used the fact that $O\left(m^{-1 / 2}\right)$-term integrates to zero and have extended the integration region with the estimate $\int_{0}^{x_{\min }}\left|\mathbf{e}_{1} \cdot \mathbf{e}_{\mathrm{n}}\right| x f(x) \mathrm{d} x \leq m^{1 / 2} c_{m} \int_{\mathbb{R}} f(x) \mathrm{d} x=O\left(m^{3 / 10}\right)$. Note that $q_{2}=Q_{2}-R \mathbf{e}_{n} \cdot \mathbf{e}_{2}$ depends on $\mathbf{e}_{n}$, but the $\mathbf{e}_{n}$-dependence annihilates as we show below in (F.7).

Substituting $x$ into $I_{2}$, we compute the diffusion coefficient to be

$$
\begin{aligned}
I_{2} & =\frac{16}{M^{4}} \int_{\mathbb{S}^{2}} \int_{x_{\min }}^{\infty}\left(\mathbf{e}_{\mathrm{n}} \otimes \mathbf{e}_{\mathrm{n}}\right)\left(\left|\mathbf{e}_{1} \cdot \mathbf{e}_{\mathrm{n}}\right| x\right)^{3} f\left(\frac{\mathbf{e}_{1} \cdot \mathbf{e}_{\mathrm{n}}}{\left|\mathbf{e}_{1} \cdot \mathbf{e}_{\mathrm{n}}\right|} x\right) \mathrm{d} x \mathrm{~d} \Omega+O\left(m^{1 / 2}\right) \\
& =\frac{16 \Phi_{3}}{M^{4}} \int_{\mathbb{S}^{2}}\left(\mathbf{e}_{\mathrm{n}} \otimes \mathbf{e}_{\mathrm{n}}\right)\left|\mathbf{e}_{1} \cdot \mathbf{e}_{\mathrm{n}}\right|^{3} \mathrm{~d} \Omega+O\left(m^{1 / 2}\right),
\end{aligned}
$$

where we have used the inequality $\int_{0}^{x_{\min }}\left(\left|\mathbf{e}_{1} \cdot \mathbf{e}_{\mathrm{n}}\right| x\right)^{3} f(x) \mathrm{d} x \leq m^{3 / 2} c_{m}^{3} \int_{\mathbb{R}} f(x) \mathrm{d} x=O\left(m^{1 / 2}\right)$.

Using the following expressions for the spherical integrals

$$
\begin{aligned}
\int_{0}^{2 \pi} \int_{0}^{\pi}\left(\mathbf{e}_{\mathrm{n}} \otimes \mathbf{e}_{\mathrm{n}}\right)\left|\mathbf{e}_{1} \cdot \mathbf{e}_{\mathrm{n}}\right| \sin \phi d \phi d \theta & =\left(\begin{array}{ccc}
\pi & 0 & 0 \\
0 & \frac{\pi}{2} & 0 \\
0 & 0 & \frac{\pi}{2}
\end{array}\right), \\
\int_{0}^{2 \pi} \int_{0}^{\pi}\left(\mathbf{e}_{\mathrm{n}} \otimes \mathbf{e}_{\mathrm{n}}\right)\left|\mathbf{e}_{1} \cdot \mathbf{e}_{\mathrm{n}}\right|^{3} \sin \phi d \phi d \theta & =\left(\begin{array}{ccc}
\frac{2 \pi}{3} & 0 & 0 \\
0 & \frac{\pi}{6} & 0 \\
0 & 0 & \frac{\pi}{6}
\end{array}\right), \\
\int_{0}^{2 \pi} \int_{0}^{\pi}\left(\mathbf{e}_{\mathrm{n}} \otimes \mathbf{e}_{\mathrm{n}}\right)\left|\mathbf{e}_{1} \cdot \mathbf{e}_{\mathrm{n}}\right|\left(\mathbf{e}_{2} \cdot \mathbf{e}_{\mathrm{n}}\right) \sin \phi d \phi d \theta & =0,
\end{aligned}
$$


we deduce from (F.5) and (F.6) that

$$
I_{1}=-\frac{16 \pi \Phi_{1}}{M^{3}}\left(\begin{array}{ccc}
1 & 0 & 0 \\
0 & \frac{1}{2} & 0 \\
0 & 0 & \frac{1}{2}
\end{array}\right)\left(\mathbf{V}-s\left(\mathbf{e}_{1} \otimes \mathbf{e}_{2}\right) \mathbf{Q}\right), \quad I_{2}=\frac{16 \pi \Phi_{3}}{M^{4}}\left(\begin{array}{ccc}
\frac{2}{3} & 0 & 0 \\
0 & \frac{1}{6} & 0 \\
0 & 0 & \frac{1}{6}
\end{array}\right) .
$$

Combining these terms with (F.3) and (F.4), writing the matrices in terms of tensor products, choosing $f$ from (2.4), and taking the limit as $m \rightarrow 0$, the generator converges in the sense of (B.2) to the generator

$$
\begin{aligned}
L \psi=\mathbf{V} & \cdot \nabla_{Q} \psi+\frac{2 \sqrt{2 \pi} \lambda R^{2}}{\sqrt{\beta} M}\left[-\left(\mathbf{e}_{1} \otimes \mathbf{e}_{1}+\frac{1}{2}\left(I-\mathbf{e}_{1} \otimes \mathbf{e}_{1}\right)\right)\left(\mathbf{V}-s\left(\mathbf{e}_{1} \otimes \mathbf{e}_{2}\right) \mathbf{Q}\right) \cdot \nabla_{V} \psi\right. \\
& \left.\left.+\frac{1}{\beta M}\left(\frac{2}{3} \mathbf{e}_{1} \otimes \mathbf{e}_{1}+\frac{1}{6}\left(I-\mathbf{e}_{1} \otimes \mathbf{e}_{1}\right)\right): \nabla_{V}^{2} \psi\right)\right]
\end{aligned}
$$

This is the generator for the anisotropic SDE

$$
\begin{aligned}
\mathrm{d} \mathbf{Q} & =\mathbf{V} \mathrm{d} t \\
M \mathrm{~d} \mathbf{V} & =-\gamma\left(\mathbf{V}-s\left(\mathbf{e}_{1} \otimes \mathbf{e}_{2}\right) \mathbf{Q}\right) \mathrm{d} t+\sigma \mathrm{d} \mathbf{W}=-\gamma(\mathbf{V}-A \mathbf{Q}) \mathrm{d} t+\sigma \mathrm{d} \mathbf{W},
\end{aligned}
$$

where

$$
\gamma=\frac{2 \sqrt{2 \pi} \lambda R^{2}}{\sqrt{\beta}}\left(\begin{array}{ccc}
1 & 0 & 0 \\
0 & \frac{1}{2} & 0 \\
0 & 0 & \frac{1}{2}
\end{array}\right) \quad \text { and } \quad \sigma=\left[\frac{4 \sqrt{2 \pi} \lambda R^{2}}{\sqrt{\beta^{3}}}\left(\begin{array}{ccc}
\frac{2}{3} & 0 & 0 \\
0 & \frac{1}{6} & 0 \\
0 & 0 & \frac{1}{6}
\end{array}\right)\right]^{1 / 2}
$$

This is the dynamics (2.12) discussed in Section 2.3. As mentioned there, we find the inconsistency of (F.8) when $s=0$ with the Langevin dynamics for zero background flow (2.1) unacceptable. As an attempt to remove the anisotropy observed in (F.8), we add laminar flows in each coordinate direction in the following section.

\section{F.2. Superimposed baths}

We now consider having three different laminar flows, one for each coordinate direction $\mathbf{e}_{i}$, for $i=1,2,3$. We define the probability distributions

$$
f_{i}(v)=Z_{i}^{-1} \exp \left(-\frac{1}{2 \theta_{i}^{2}} v^{2}\right), \quad \text { where } Z_{i}=\sqrt{2 \pi} \theta_{i}, \quad i=1,2,3,
$$

where we allow for different temperatures for each of the bath measures. The initial condition of the bath atoms is chosen according to the measure

$$
\begin{aligned}
\mathrm{d} \mu_{m}(\mathbf{q}, \mathbf{v}) & =\frac{\lambda_{m} m^{1 / 2}}{3}\left(f_{1}\left(m^{1 / 2}\left(v_{1}-s q_{2}\right)\right) \delta\left(v_{2}\right) \delta\left(v_{3}\right)\right. \\
& \left.+f_{2}\left(m^{1 / 2} v_{2}\right) \delta\left(v_{1}\right) \delta\left(v_{3}\right)+f_{3}\left(m^{1 / 2} v_{3}\right) \delta\left(v_{1}\right) \delta\left(v_{2}\right)\right) \mathrm{d} \mathbf{v d} \mathbf{q}, \quad \mathbf{q}, \mathbf{v} \in \mathbb{R}^{3},
\end{aligned}
$$

where $\delta(x)$ is the Dirac distribution.

Upon splitting the integral linearly, the calculations of Appendix F.1 give us the limiting generator

$$
L \psi=\mathbf{V} \cdot \nabla_{Q} \psi-M^{-1}(\gamma \mathbf{V}-\tilde{u}) \cdot \nabla_{V} \psi+\frac{1}{2} M^{-2} \sigma \sigma^{T}: \Delta_{V} \psi
$$


where

$$
\begin{aligned}
\gamma & =\frac{2 \sqrt{2 \pi} \lambda R^{2}}{3}\left(\begin{array}{ccc}
\theta_{1}+\frac{1}{2} \theta_{2}+\frac{1}{2} \theta_{3} & 0 & 0 \\
0 & \frac{1}{2} \theta_{1}+\theta_{2}+\frac{1}{2} \theta_{3} & 0 \\
0 & 0 & \frac{1}{2} \theta_{1}+\frac{1}{2} \theta_{2}+\theta_{3}
\end{array}\right), \\
\tilde{u} & =\frac{2 \sqrt{2 \pi} \lambda R^{2}}{3}\left(\begin{array}{c}
s Q_{2} \theta_{1} \\
0 \\
0
\end{array}\right), \\
\sigma \sigma^{T} & =\frac{4 \sqrt{2 \pi} \lambda R^{2}}{3}\left(\begin{array}{ccc}
\frac{2}{3} \theta_{1}^{3}+\frac{1}{6} \theta_{2}^{3}+\frac{1}{6} \theta_{3}^{3} & 0 & 0 \\
0 & \frac{1}{6} \theta_{1}^{3}+\frac{2}{3} \theta_{2}^{3}+\frac{1}{6} \theta_{3}^{3} & 0 \\
0 & 0 & \frac{1}{6} \theta_{1}^{3}+\frac{1}{6} \theta_{2}^{3}+\frac{2}{3} \theta_{3}^{3}
\end{array}\right) .
\end{aligned}
$$

The corresponding SDE is

$$
\begin{aligned}
\mathrm{d} \mathbf{Q} & =\mathbf{V} \mathrm{d} t \\
M \mathrm{~d} \mathbf{V} & =-\gamma \mathbf{V} \mathrm{d} t+\tilde{u} \mathrm{~d} t+\sigma \mathrm{d} \mathbf{W} .
\end{aligned}
$$

As an example, let us take $\theta_{i}=\frac{1}{\sqrt{\beta}}$ for $i=1,2,3$. Then we have for the limiting equation

$$
\begin{aligned}
\mathrm{d} \mathbf{Q} & =\mathbf{V} \mathrm{d} t \\
M \mathrm{~d} \mathbf{V} & =-\gamma\left(\mathbf{V}-\frac{1}{2} s Q_{2} \mathbf{e}_{1}\right) \mathrm{d} t+\left(\frac{\gamma}{\beta}\right)^{1 / 2} \mathrm{~d} \mathbf{W},
\end{aligned}
$$

where $\gamma=\frac{4 \lambda R^{2} \sqrt{2 \pi}}{3 \sqrt{\beta}}$. This is (2.15), which is discussed in Section 2.3. This system satisfies the fluctuationdissipation relation only for inverse temperature $\tilde{\beta}=2 \beta$. That is, the temperature of the large particle is half that of the bath. More problematically, there is a factor of $1 / 2$ on $s$, which means that the large particle only feels half the average velocity of the heat bath. This is caused by the superposition of multiple laminar flows. We note that there is no choice of $\theta_{1}, \theta_{2}$, and $\theta_{3}$ so that the resulting SDE is both isotropic and has response $\tilde{u}$ equal to the input background motion.

Acknowledgements. This work is supported in part by the NSF Mathematical Sciences Postdoctoral Research Fellowship and by the Agence Nationale de la Recherche, under grants ANR-06-CIS6-006 (PARMAT) and ANR-09-BLAN-0216-01 (MEGAS). The authors would like to thank Claude Le Bris for many fruitful discussions.

\section{REFERENCES}

[1] M.P. Allen and D.J. Tildesley, Computer simulation of liquids. Clarendon Press, New York, NY, USA (1989).

[2] P. Billingsley, Convergence of probability measures. Wiley Series in Probability and Statistics: Probability and Statistics. John Wiley and Sons Inc., New York, second edition (1999).

[3] P. Calderoni, D. Dürr and S. Kusuoka, A mechanical model of Brownian motion in half-space. J. Stat. Phys. 55 (1989) $649-693$.

[4] G. Ciccotti, R. Kapral and A. Sergi, Non-equilibrium molecular dynamics. In Handbook of Materials Modeling, edited by S. Yip (2005) $745-761$.

[5] R. Cont and P. Tankov, Financial modelling with jump processes. Chapman \& Hall/CRC Financial Mathematics Series. Chapman \& Hall/CRC, Boca Raton, FL (2004).

[6] D. Dür, S. Goldstein and J. Lebowitz, A mechanical model for the Brownian motion of a convex body. Z. Wahrscheinlichkeit 62 (1983) 427-448.

[7] D. Dürr, S. Goldstein and J.L. Lebowitz. A mechanical model of Brownian motion. Comm. Math. Phys. 78 (1981) 507-530.

[8] B. Edwards, C. Baig and D. Keffer, A validation of the p-SLLOD equations of motion for homogeneous steady-state flows. J. Chem. Phys. 124 (2006).

[9] D.J. Evans and G.P. Morriss, Statistical mechanics of nonequilibrium liquids. ANU E Press, Canberra (2007).

[10] N.G. Hadjiconstantinou, Discussion of recent developments in hybrid atomistic-continuum methods for multiscale hydrodynamics. Bull. Pol. Acad. Sci-Te. 53 (2005) 335-342. 
[11] J.H. Irving and J.G. Kirkwood, The statistical mechanical theory of transport processes. IV. The equations of hydrodynamics. J. Chem. Phys. 18 (1950) 817-829.

[12] R. Joubaud and G. Stoltz, Nonequilibrium shear viscosity computations with Langevin dynamics. Multiscale Model. Simul. 10 (2012) 191-216.

[13] P. Kotelenez, Stochastic ordinary and stochastic partial differential equations. In vol. 58 of Stoch. Modell. Appl. Probab. (2008).

[14] T.G. Kurtz, Semigroups of conditioned shifts and approximation of Markov processes. Ann. Probab. 3 (1975) 618-642.

[15] S. Kusuoka and S. Liang, A Classical Mechanical Model of Brownian Motion with Plural Particles. Rev. Math. Phys. 22 (2010) 733-838.

[16] C. Le Bris and T. Lelièvre, Micro-macro models for viscoelastic fluids: modelling, mathematics and numerics. Sci. China Math. 55 (2012) 353-384.

[17] F. Legoll, M. Luskin and R. Moeckel, Non-ergodicity of the Nosé-Hoover thermostatted harmonic oscillator. Arch. Ration. Mech. Anal. 184 (2007) 449-463.

[18] F. Legoll, M. Luskin and R. Moeckel, Non-ergodicity of Nosé-Hoover dynamics. Nonlinearity 22 (2009) $1673-1694$.

[19] M. McPhie, P. Daivis, I. Snook, J. Ennis and D. Evans, Generalized Langevin equation for nonequilibrium systems. Phys. A 299 (2001) 412-426.

[20] S.T. O'Connell and P.A. Thompson, Molecular dynamics-continuum hybrid computations: A tool for studying complex fluid flows. Phys. Rev. E 52 (1995) R5792-R5795.

[21] W. Ren and W. E, Heterogeneous multiscale method for the modeling of complex fluids and micro-fluidics. J. Comput. Phys. 204 (2005) 1-26.

[22] R. Rowley and M. Painter, Diffusion and viscosity equations of state for a Lennard-Jones fluid obtained from molecular dynamics simulations. Int. J. Thermophys. 18 (1997) 1109-1121.

[23] A.V. Skorokhod, Limit theorems for Markov processes. Theor. Probab. Appl. 3 (1958) 202-246.

[24] T. Soddemann, B. Dünweg and K. Kremer, Dissipative particle dynamics: A useful thermostat for equilibrium and nonequilibrium molecular dynamics simulations. Phys. Rev. E 68 (2003) 046702.

[25] B. Todd and P.J. Daivis, A new algorithm for unrestricted duration nonequilibrium molecular dynamics simulations of planar elongational flow. Comput. Phys. Commun. 117 (1999) 191-199.

[26] B.D. Todd and P.J. Daivis, Homogeneous non-equilibrium molecular dynamics simulations of viscous flow: techniques and applications. Mol. Simulat. 33 (2007) 189-229.

[27] M.E. Tuckerman, C.J. Mundy, S. Balasubramanian and M.L. Klein, Modified nonequilibrium molecular dynamics for fluid flows with energy conservation. J. Chem. Phys. 106 (1997) 5615-5621.

[28] T. Werder, J.H. Walther and P. Koumoutsakos, Hybrid atomistic-continuum method for the simulation of dense fluid flows. J. Comp. Phys. 205 (2005) 373-390. 